\title{
OPTIMIZATION OF SPECTRUM SENSING SCHEMES IN COGNITIVE SENSOR NETWORKS
}

by

\author{
Amir Sepasi Zahmati \\ BSc, K.N. Toosi University of Technology, Tehran, Iran, 2004 \\ MSc, Iran University of Science and Tech., Tehran, Iran, 2007
}

\author{
A dissertation \\ presented to Ryerson University \\ in partial fulfillment of the \\ requirements for the degree of \\ Doctor of Philosophy \\ in the Program of \\ Electrical and Computer Engineering
}

Toronto, Ontario, Canada, 2013

(C)Amir Sepasi Zahmati 2013 
I hereby declare that I am the sole author of this dissertation. This is a true copy of the dissertation, including any required final revisions, as accepted by my examiners.

I authorize Ryerson University to lend this dissertation to other institutions or individuals for the purpose of scholarly research.

I further authorize Ryerson University to reproduce this dissertation by photocopying or by other means, in total or in part, at the request of other institutions or individuals for the purpose of scholarly research.

I understand that my dissertation may be made electronically available to the public. 
Optimization of Spectrum Sensing Schemes in Cognitive Sensor Networks

Doctor of Philosophy 2013

\author{
Amir Sepasi Zahmati \\ Electrical and Computer Engineering \\ Ryerson University
}

\begin{abstract}
The currently dominant spectrum allocation policy is reported to be inefficient. Cognitive radio, therefore, has been proposed in the literature to improve the spectrum usage efficiency. This dissertation proposes the optimization of spectrum sensing schemes in cognitive sensor networks. The modeling of the spectrum occupancy is a prerequisite for cognitive radio analysis. We describe the radio spectrum occupancy as a continuoustime Markov chain, and mathematically define the model by deriving the transition rate matrix and the probability state vector.
\end{abstract}

The dissertation addresses an important aspect of spectrum sensing that has been often overlooked in the literature. While the cognitive radio is supposed to be aware of its surroundings, existing work does not consider the characteristics of unlicensed users for finding the optimum sensing period. In this work, we propose an applicationspecific method that finds the optimal sensing period according to the characteristics of both secondary and primary networks. According to the unlicensed user's state in the Markov chain, two optimization problems are formulated to derive the optimum sensing 
periods. The secondary network's throughput and power consumption are also studied and the corresponding parameters are derived. By numerical and simulation analyses, it is elaborated that the proposed method increases the secondary network's throughput by up to $11 \%$ and significantly decreases the power consumption of the secondary network by as low as $33 \%$ of the non-hybrid approach.

In addition, we study cooperative spectrum sensing in cognitive sensor networks and address two important issues. First, an optimization problem is solved to obtain the minimum required number of cognitive users. Second, we define a metric for sensing accuracy and propose a novel energy-aware secondary user selection method that identifies the most eligible cognitive users through a probability-based approach. The network's lifetime is compared at several sensing accuracy thresholds and the trade-off between sensing accuracy and network lifetime is studied. Finally, the effects of several fusion rules on the proposed method are studied through simulation and numerical analyses. It is discussed that the Majority rule has the best performance among the examined rules. 


\section{Acknowledgements}

This research project would not have been possible without the support of many people. I would like to express my deepest gratitude to my Ph.D. supervisor, Prof. Xavier Fernando, for his support, creative advising, and guidance throughout this work. I especially acknowledge Dr. Fernando's encouragement to think out of the box, which led me to the opportunity to be a Research Intern at Honeywell Aerospace during 20102011. I would like to extend my deepest gratitude and appreciation to Dr. Hassan Kojori for his invaluable guidance, background knowledge, and continued support since the beginning of my internship at Honeywell Aerospace.

I would also like to recognize Dr. Ali Grami, my Ph.D. co-supervisor, for his guidance, patience and editing skills in the completion of this dissertation. Prof. Grami read my numerous revisions, and helped make some sense of the confusion. Dr. Grami's insight, in particular, directed me towards the selection of my Ph.D. research area.

To the other members of my committee, Dr. Jamal Deen, Dr. Kaamran Raahemifar and Dr. Matthew Kyan, thank you for the feedback, direction, and assistance when I needed it. Special thanks to my friends and colleagues in the Ryerson Communications Lab (RCL) research group for their insightful discussions and professional collaborations.

I would also like to acknowledge my brother, Hamid Sepasy Zahmaty, and his wife, Banafsheh Heydarzadeh, for their continued support and endless love. There is no doubt in my mind that without their help I could not have completed this long process. 


\section{Dedication}

This Doctoral dissertation is lovingly dedicated to my late father, Abbas, and my mother, Maliheh, for their unconditional love, sacrifice, hard work and strength. My mother, in particular, always had confidence in me and offered me encouragement and support in all my endeavours; I owe her every single accomplishment in my life. 


\section{Table of Contents}

Abstract $\ldots \ldots \ldots \ldots \ldots$
Acknowledgements $\ldots \ldots \ldots \ldots \ldots \ldots \ldots$

1 Introduction $\quad 1$

1.1 CR Research Challenges . . . . . . . . . . . . . . . . . . 2

1.2 Major Contributions of the Dissertation _. . . . . . . . . . . 4

1.3 Organization of the Dissertation . . . . . . . . . . . . 5

2 Cognitive Radio Technology $\quad 8$

2.1 Basic Concepts . . . . . . . . . . . . . . . . . . . . . . 8

2.2 Cognitive Sensor Networks . . . . . . . . . . . . . . . . . . 10

2.3 Spectrum Sensing . . . . . . . . . . . . . . . . . . . . . 11

2.4 Recent Advancements . . . . . . . . . . . . . . . . . . . . . . 16

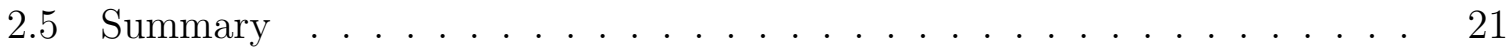

3 Spectrum Occupancy Model 23

3.1 Background . . . . . . . . . . . . . . . . . . . . . . . . . 23

3.2 System Model . . . . . . . . . . . . . . . . . . . . . . . . 28

3.3 Derivation of the Steady-State Probabilities . . . . . . . . . . 30

3.4 Numerical Analysis . . . . . . . . . . . . . . . . . . . . 35

3.4 .1 No Active Secondary User $(N=0) \ldots \ldots \ldots \ldots$ 
3.4 .2 Three Secondary Users $(N=3) \ldots \ldots \ldots \ldots \ldots$

3.4.3 Very Large Number of Secondary Users . . . . . . . . . . . . . . 41

3.5 Summary . . . . . . . . . . . . . . . . . . . . . . . . . 42

4 Application-Specific Spectrum Sensing 44

4.1 Motivations . . . . . . . . . . . . . . . . . . . . . . 44

4.2 Energy Detection Method . . . . . . . . . . . . . . . . . . 46

4.3 Application-Specific Spectrum Sensing . . . . . . . . . . . . . . 49

4.3 .1 Problem Definitions . . . . . . . . . . . . . . . . 51

$4.3 .2 \quad$ Derivation of $R_{I}$ and $R_{U} \ldots \ldots \ldots \ldots \ldots \ldots \ldots$

4.3 .3 Throughput Analysis . . . . . . . . . . . . . . . . . . . 59

4.3.4 Energy Consumption Analysis . . . . . . . . . . . . . . . . . . 62

4.3.5 Problem Formulation . . . . . . . . . . . . . . . . . . 63

4.3.5.1 Secondary Network Transmission State . . . . . . . . . 64

4.3.5.2 Secondary Network Idle State . . . . . . . . . . . . . 65

4.3.5.3 Optimum Sensing Period . . . . . . . . . . . 66

4.4 Non-Application-Specific Spectrum Sensing . . . . . . . . . . . . . 66

4.5 Numerical and Simulation Results . . . . . . . . . . . . . . . . . . . 69

4.5.1 Interference Ratio Analysis . . . . . . . . . . . . . . . . . 69

4.5.2 Undetected Opportunity Ratio Analysis . . . . . . . . . . . . 70

4.5.3 Optimum Sensing Period Analysis . . . . . . . . . . . . . . . 72

4.5.4 Throughput and Power Consumption Analysis . . . . . . . . . . 74

4.6 Summary . . . . . . . . . . . . . . . . . . . . . . . . . . . 78

5 Energy-Aware Secondary User Selection in Cooperative Spectrum Sensing $\quad 80$

5.1 Motivations . . . . . . . . . . . . . . . . . . . . . 80

5.2 Cooperative Spectrum Sensing . . . . . . . . . . . . . . . . . . . . . 83

5.3 Energy-Aware Cognitive User Selection . . . . . . . . . . . . . . . 86

5.3.1 Minimum Number of Required Cognitive Users in the Subset . . . 86

5.3 .2 Identification of the Most Eligible SUs . . . . . . . . . . 90

5.3 .3 Evaluation Benchmarks . . . . . . . . . . . . . 97

5.4 Numerical and Simulation Results . . . . . . . . . . . . . . . . . . 97

5.4.1 The Secondary Network's Lifetime . . . . . . . . . . . . . . 101

5.4 .2 The Fusion Rule's Effect . . . . . . . . . . . . . . . . . . . . . . 103 


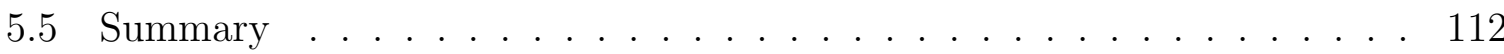

6 Conclusions and Future Work $\quad 114$

6.1 Concluding Remarks . . . . . . . . . . . . . . . . . . . . . . 114

6.2 Future Directions . . . . . . . . . . . . . . . . . . . 117

$\begin{array}{ll}\text { References } & 119\end{array}$ 


\section{List of Tables}

2.1 A brief history of CR-like communication systems. . . . . . . . . . . 9

3.1 The proposed model at $N=0$ agrees with the existing model in [1]. . . . 37

4.1 A few examples of sensor networks in the WAIC project. . . . . . . . . 45

4.2 Simulation parameters for the throughput and power consumption analyses. 76

5.1 Emphasizing the sensing accuracy vs. the secondary network's energy. . . 100

5.2 Simulation parameters. . . . . . . . . . . . . . . . . . 102

5.3 Classification of the secondary users into three categories. . . . . . . . . 103 


\section{List of Figures}

1.1 Local spectrum sensing vs. cooperative spectrum sensing. . . . . . . . . . 4

1.2 Inter-relationship diagram between the dissertation chapters. . . . . . . . 6

2.1 Different states in a WSN vs. a CSN. . . . . . . . . . . . . . . . . 11

2.2 Classification of spectrum sensing methods. . . . . . . . . . . . . . 13

2.3 Receiver uncertainty and shadowing uncertainty problems in the transmitter detection method. . . . . . . . . . . . . . . . . . 17

3.1 A CTMC approximation to model the primary channel usage pattern. . . 25

3.2 A CR network with mobile SUs. . . . . . . . . . . . . . . . 26

3.3 A three-state CTMC system to model the mobility-aware channel availability experienced by SUs. . . . . . . . . . . . . . . . 26

3.4 A Markov chain model for CR; $i$ is the total number of sub-bands used by SUs, and $j$ is the total number of primary bands used by the PU [2]. . . 28

3.5 The state transition rate diagram for the proposed CTMC model. . . . . 31

3.6 The state transition diagram in: (a) our model at $N=0$, (b) the existing model in [1]. . . . . . . . . . . . . . . . . . . . . 37

3.7 The state transition rate diagram for $N=3 \ldots \ldots \ldots$

3.8 The stationary state probabilities for cases (a), (b), and (c). . . . . . . 40

3.9 The stationary state probabilities in a secondary network with $N=100$ SUs. . . . . . . . . . . . . . . . . . . . . 42

4.1 Non-application-specific spectrum sensing vs. application-specific spectrum sensing in CR. The PU's properties are assumed to be the same for the two types of applications. . . . . . . . . . . . . . . 47

4.2 The operation of the CSN is divided into three states: idle, secondary network transmission, and primary user transmission. . . . . . . . . 51 
4.3 Illustration of cases of interference, undetected spectrum opportunity, and interference-free transmission time in the hybrid spectrum sensing. . . . .

4.4 Illustration of the events of interference, undetected spectrum opportunity, and interference-free transmission time in the non-hybrid spectrum sensing. 68

4.5 Interference ratio for the secondary network 1, 2, and $3\left(\Pi_{s 1}<\Pi_{s 2}<\Pi_{s 3}\right.$ and $\left.R_{I}^{t h}=0.2\right) \ldots \ldots \ldots \ldots \ldots \ldots \ldots \ldots \ldots \ldots \ldots \ldots \ldots \ldots \ldots \ldots \ldots$

4.6 Undetected opportunity ratio for the secondary network 1 , 2 , and $3\left(\Pi_{s 1}<\right.$

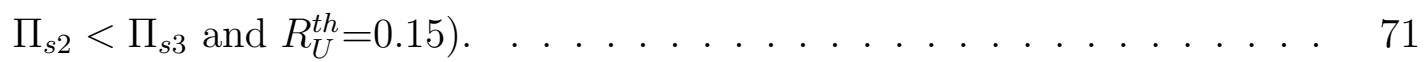

4.7 The average of the optimum sensing period in Case-I. . . . . . . . . . 73

4.8 The average of the optimum sensing period in Case-II. . . . . . . . . . 75

4.9 The average throughput of the secondary networks (theoretical results). . 75

4.10 Total power consumption of the secondary networks (theoretical results). $\quad 77$

4.11 The secondary network's average throughput, simulation analysis. . . . . 77

4.12 The secondary network's power consumption, simulation analysis. . . . . 78

5.1 Cooperative spectrum sensing in a CSN . . . . . . . . . . . . 84

5.2 The proposed method's timeline. . . . . . . . . . . . . . . . . 90

5.3 The proposed method's flowchart; $r$ denotes the current round, $c_{-}$setup and $c_{-} o p$ are the current number of rounds in the setup and operation phases respectively, and epoch is the number of rounds in an epoch. . . .

5.4 Check-Dead: a subroutine to check the number of dead SUs. $n_{\text {dead }}$ and $n_{\text {alive }}$ are the number of dead and alive SUs respectively. . . . . . . 95

5.5 Minimum number of required cooperative users as a function of $\varsigma_{f},\left(\varsigma_{d}=\right.$ $0.85) \ldots \ldots \ldots \ldots \ldots \ldots \ldots$

5.6 Minimum number of required cooperative users as a function of $\varsigma_{d},\left(\varsigma_{f}=0.2\right) .99$

5.7 The effect of the number of rounds within the setup phase $(W) \ldots \ldots$

5.8 The accuracy and energy benchmarks in Case-A and Case-B. . . . . . . . 102

5.9 The secondary network's lifetime at different values of $\Delta_{t h} \ldots \ldots \ldots$. . . 104

5.10 Total number of times each secondary user is selected at $\Delta_{t h}=0$ and $\Delta_{t h}=4 \ldots \ldots \ldots \ldots \ldots \ldots \ldots \ldots \ldots \ldots$

5.11 The probability of interference $P_{I}$ for different fusion rules. . . . . . . 106

5.12 The probability of missed opportunities $P_{m}$ for different fusion rules. . . . 106

5.13 The probability of error $P_{e}$ for different fusion rules. . . . . . . . . 108 
5.14 The probability of error $P_{e}$ for different values of $k$ in the k-out-of-N rule $(1 \leq k \leq 10)$. The minimum required number of cooperative users is considered to be $N^{*}=10 \ldots \ldots \ldots$. . . . . . . . . . . . . 108

5.15 The operation of the proposed method is divided into the setup phase and the operational phase. An "X" indicates that the corresponding SU does not perform senseing/reporting, a "1" means the PU is detected, and a "0" means the PU is not detected. . . . . . . . . . . . . . . . . 110

5.16 Illustration of the weighting factor $\eta$, the probability matrix $P$, and the energy consumption matrix $e$ for rounds $1 \leq r \leq 10$. An "** in $P$ matrix indicates the active SUs at each round. . . . . . . . . . . . . 


\title{
List of Acronyms
}

\author{
AWGN Additive White Gaussian Noise \\ CR Cognitive Radio \\ CSN Cognitive Sensor Network \\ CTMC Continuous-Time Markov Chain \\ DSA Dynamic Spectrum Access \\ FC Fusion Centre \\ FCC Federal Communications Commission \\ HD High-Definition \\ HMM Hidden Markov Model \\ IID Independent and Identically Distributed \\ ISM Industrial, Scientific and Medical band \\ MAC Medium Access Control \\ NBP National Broadband Plan \\ PDF Probability Density Function \\ PPR Primary Protection Region \\ PU Primary User \\ RF Radio Frequency \\ SNR Signal-to-Noise Ratio \\ SSP Stationary State Probability \\ SU Secondary User \\ TVWS TV White Spaces \\ WRAN Wireless Regional Area Network \\ WSN Wireless Sensor Network
}




\section{List of Symbols}

$\Delta_{i}(n)$ Total number of differences between the $i^{\text {th }}$ secondary user's local sensing and the FC's global decision, during the setup phase

$\Delta_{t h} \quad$ Sensing accuracy threshold

$D_{i}(n) \quad$ The result of local sensing performed by the $i^{\text {th }}$ secondary user, during the $n^{\text {th }}$ sensing task

$e_{0} \quad$ Energy consumption to perform a single sensing/reporting task

$e_{u} \quad$ Initial energy of secondary users

$E \quad$ Total energy consumption of the secondary network

$\epsilon \quad$ Decision threshold to decide on the presence or absence of the PU

$\eta_{i} \quad$ Weighting factor assigned to the $i^{\text {th }}$ secondary user

$F(n) \quad$ The result of the FC's decision at the $n^{\text {th }}$ round

$\Gamma_{s} \quad$ Average throughput of the secondary network $s$

$\gamma_{d} \quad$ Global probability of detection threshold

$\gamma_{f} \quad$ Global probability of false alarm threshold

$k_{i}(t) \quad$ Channel gain of the sensing channel between the $i^{t h} \mathrm{SU}$ and the PU

$L \quad$ Number of rounds within the operational phase

$\lambda_{P} \quad$ PU's arrival rate

$\lambda_{i} \quad$ The $i^{\text {th }}$ secondary user's arrival rate

$M \quad$ Number of received samples

$\mu_{P} \quad$ PU's departure rate

$\mu_{i} \quad$ The $i^{t h}$ secondary user's departure rate

$n_{i}(t) \quad$ Additive white Gaussian noise at the $i^{t h}$ secondary user

$N \quad$ Number of secondary users

$N^{*} \quad$ Minimum number of required secondary users

$P \quad$ Total power consumption of the secondary network 


$\begin{array}{ll}P_{f} & \text { Probability of false alarm } \\ P_{d} & \text { Probability of detection } \\ \Pi_{i} & \text { The steady-state probability of being at state } i \\ Q_{d} & \text { Global probability of detection } \\ Q_{f} & \text { Global probability of false alarm } \\ r_{i}(t) & \text { Received signal at the } i^{\text {th }} \text { secondary user } \\ r_{i} & \text { Maximal data rate of the } i^{\text {th }} \text { secondary user } \\ R_{I} & \text { Interference ratio } \\ R_{I}^{t h} & \text { Interference ratio threshold } \\ R_{I}^{N H} & \text { Interference ratio in the non-hybrid method } \\ R_{U} & \text { Undetected opportunity ratio } \\ R_{U}^{t h} & \text { Undetected opportunity ratio threshold } \\ R_{U}^{N H} & \text { Undetected opportunity ratio in the non-hybrid method } \\ s(t) & \text { Primary user transmitted signal } \\ \sigma_{n}^{2} & \text { Variance of the noise } \\ \sigma_{s}^{2} & \text { Variance of the transmitted signal } \\ T_{s i m} & \text { Simulation time } \\ T_{I}^{*} & \text { Optimal idle time } \\ T_{T}^{*} & \text { Optimal transmission time } \\ T_{O} & \text { Observation time } \\ \varsigma_{d} & \text { Probability of detection threshold } \\ \varsigma_{f} & \text { Probability of false alarm threshold } \\ W & \text { Number of rounds within the setup phase } \\ Y & \text { Test statistic in the energy detection method }\end{array}$




\section{Chapter 1}

\section{Introduction}

Currently, the radio spectrum is mainly used based on a fixed assignment policy, where either the spectrum is allocated to licensed service providers, such as TV bands, or it is open to the public for use with an agreed-upon etiquette, such as the ISM band. While the fixed allocation policy dominates the current wireless systems, it is inefficient [3]. For instance, a spectrum usage measurement by the US Federal Communications Commission (FCC) indicates that a large portion of the assigned radio spectrum is severely underutilized, while the demand for the radio spectrum is rapidly increasing [4].

The usable portion of the radio spectrum is limited; therefore, dynamic spectrum access (DSA) has gained momentum in both academia and industry to overcome the issue of spectrum scarcity. In particular, cognitive radio (CR), an increasingly developing technology, has been viewed as a promising approach to tackle this concern [5]. 
A CR is an intelligent wireless communication system that is aware of its surroundings, and adapts its internal parameters to achieve a reliable communication and efficient utilization of the spectrum $[6,7]$. Through CR technology, the vacant portions of the radio spectrum that is originally assigned to licensed/primary users (PUs) can be opportunistically utilized by unlicensed/secondary users (SUs). A band of frequencies that is originally assigned to PUs but is temporarily vacant and thus can be used by SUs, is called a spectrum hole or a white space.

\section{$1.1 \quad$ CR Research Challenges}

Through CR technology, the radio spectrum can be utilized by both licensed and unlicensed users. Developing analytical models that consider the occupancy of the radio spectrum by the two types of users, therefore, is listed as one of the major research challenges in $\mathrm{CR}$. It is important to understand the theory behind CR algorithms and to evaluate the performance of different algorithms.

As a CR network should be aware of its surroundings, spectrum sensing has been considered as an important necessity for the realization of cognitive technology. Through spectrum sensing, SUs monitor the primary network's activity to opportunistically use the spectrum and to vacate the spectrum in cases where a PU appears. Several challenges

are associated with the task of spectrum sensing. In a group of sensing methods, non- 
cooperative spectrum sensing, the sensing task is performed by each SU individually. In other words, there is no collaboration between different SUs. In such approaches, it is important to obtain the optimal sensing frequency in order to achieve a predetermined objective, such as maximization of the secondary network's throughput.

Through another group of sensing methods, cooperative spectrum sensing, SUs do collaborate to perform the sensing task. In Fig. 1.1, the non-cooperative (local) spectrum sensing is compared with the cooperative sensing scheme. As shown, in the local sensing scheme, each sensor must have the ability to sense the whole channel and determine an optimal scheme to maximize its benefits, such as its throughput. However, in the cooperative sensing scheme, each SU senses the spectrum, and reports its local sensing result to a single entity called a fusion centre (FC). The FC gathers all local sensing results, and makes a final decision on the presence or absence of the PU. The final decision is then forwarded to all SUs.

In cooperative spectrum sensing, design challenges include but are not limited to finding the minimum number of required cooperative users, and choosing the most qualified SUs in terms of energy level and sensing accuracy. 

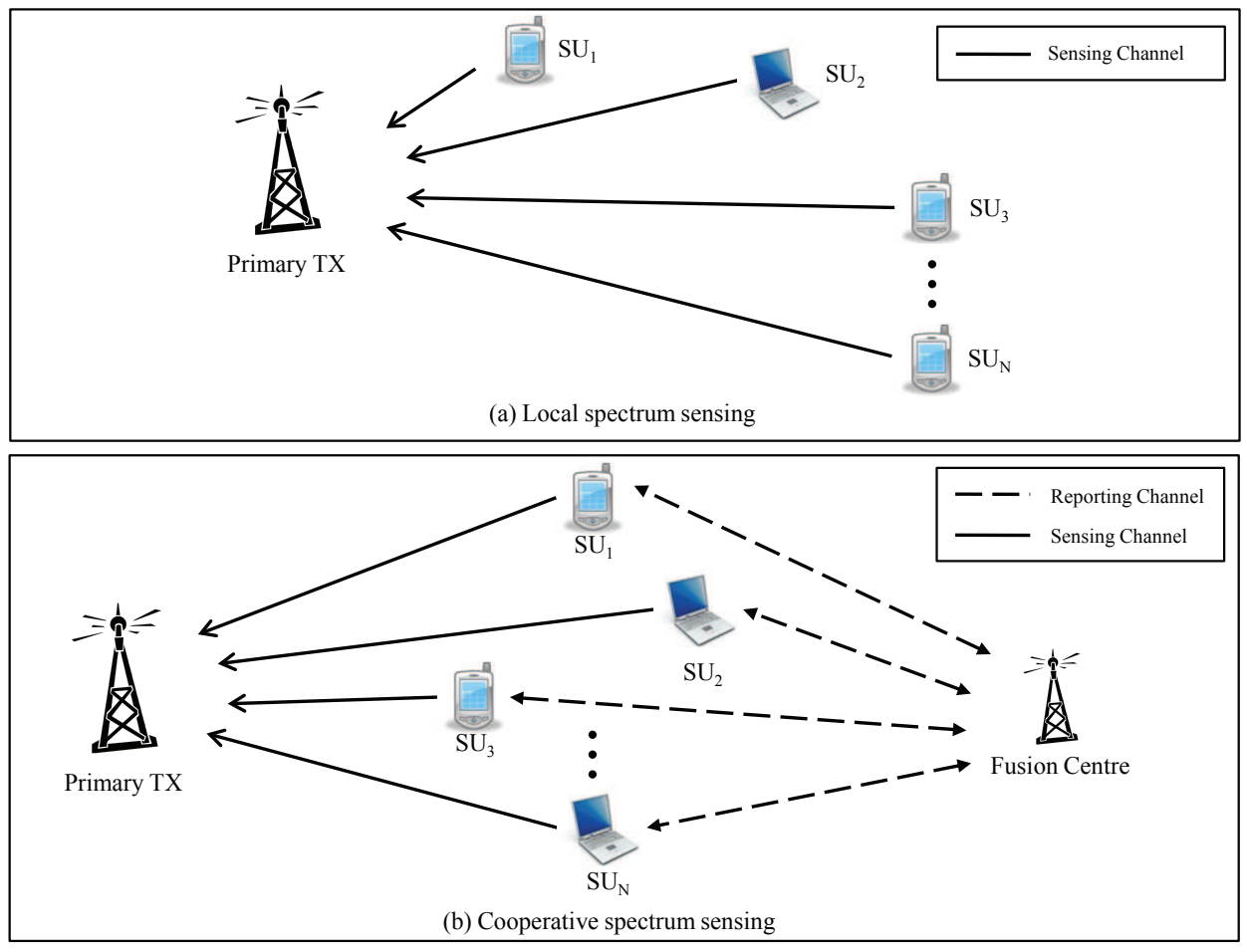

Figure 1.1: Local spectrum sensing vs. cooperative spectrum sensing.

\subsection{Major Contributions of the Dissertation}

In this dissertation, we use a continuous-time Markov chain (CTMC) to describe the spectrum occupancy by both primary and secondary networks. By analytical work, we obtain the transition rate matrix for the proposed model. In addition, we perform steady-state analysis to analytically derive the probability state vector. The CTMC is then employed to serve as a base model for further analysis and proposals in the rest of this work.

In addition, we address the problem of local spectrum sensing optimization in CR from a novel perspective. In contrast with a majority of existing work, where the sensing 
optimization is performed according to the properties of the primary network only, we propose an application-specific ${ }^{1}$ sensing method that finds the SUs' sensing period according to the properties of both primary and secondary networks. Moreover, the interference and the undetected opportunity ratios are derived, and two optimization problems are formulated to find the optimum sensing period, based on the CTMC model presented in this work. In addition, the non-interfered system throughput and the total power consumption of the secondary network are derived. We also derive the corresponding parameters in a non-application-specific ${ }^{2}$ method for comparison purposes.

Furthermore, we apply cooperative sensing in CR to further enhance the performance of spectrum sensing. First, we analytically obtain the minimum number of cooperative users needed to perform the sensing task, while meeting the sensing requirements. Second, the most eligible SUs are identified based on a probability-based approach that utilizes a sensing accuracy metric and a weighting function. Figure 1.2 shows the interrelationship between different chapters of this dissertation.

\subsection{Organization of the Dissertation}

The dissertation is established in six chapters, as follows:

\footnotetext{
${ }^{1}$ The terms "application-specific" and "hybrid" will be used interchangeably in this dissertation.

${ }^{2}$ The terms "non-application-specific" and "non-hybrid" will be used interchangeably in this dissertation.
} 


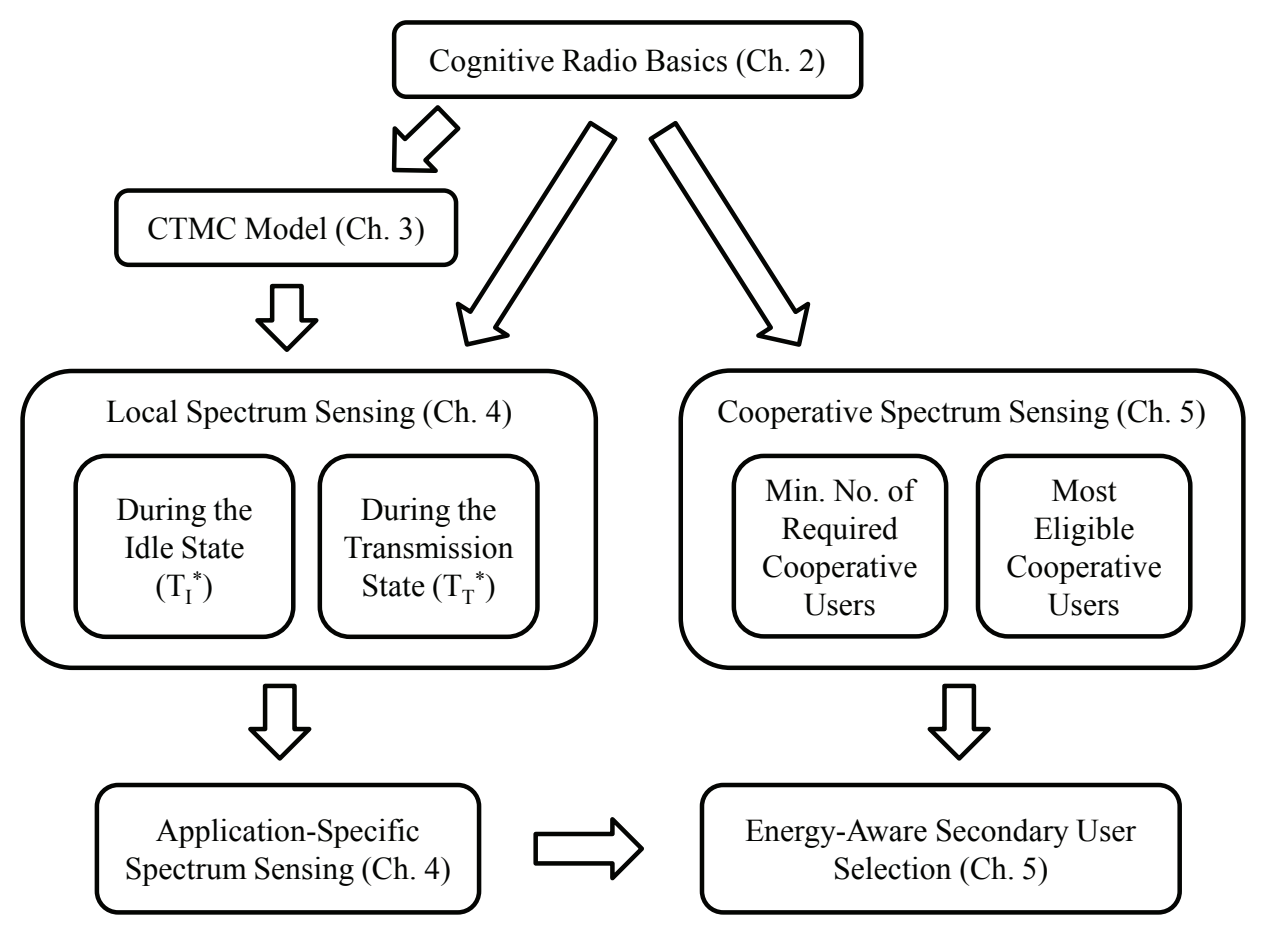

Figure 1.2: Inter-relationship diagram between the dissertation chapters.

\section{Chapter 1: Introduction}

In this chapter, a brief introduction to the dissertation is presented. Major research challenges are discussed along with our major contributions to overcome the challenges.

\section{Chapter 2: Cognitive Radio Technology}

A short background of CR technology is presented in this chapter. We address spectrum sensing as an important necessity in the realization of cognitive systems and study recent advancements in the field.

\section{Chapter 3: Spectrum Occupancy Model}

This chapter studies a CTMC system to accurately model the spectrum occupancy by 
the primary and secondary networks in CR. The system will serve as a base model for our proposals through the dissertation.

\section{Chapter 4: Application-Specific Spectrum Sensing}

An application-specific sensing method is proposed in this chapter to provide a customized solution for various secondary applications. The proposed method is a hybrid approach that considers the properties of both primary and secondary networks to obtain optimal sensing period.

Chapter 5: Energy-Aware Secondary User Selection in Cooperative Spectrum

\section{Sensing}

In this chapter, we apply cooperative spectrum sensing to further improve the sensing performance. An energy-aware secondary user selection method is proposed that accurately obtains the minimum number of required secondary users to minimize the secondary network's energy consumption. In addition, the proposed method sets a merit for sensing accuracy based on recent sensing results, and selects the most eligible users through a probability-based approach. The selected secondary users then sense the licensed channel and report their sensing results to a fusion centre.

\section{Chapter 6: Conclusions and Future Work}

This chapter presents the concluding remarks of this work, and some suggestions for future research are made. 


\section{Chapter 2}

\section{Cognitive Radio Technology}

\section{$2.1 \quad$ Basic Concepts}

Cognitive radio is recognized by two main characteristics: cognitive capability and reconfigurability. A CR system can identify and exploit the unused portions of the spectrum without interference with the licensed users, through real-time interaction with the radio environment (cognitive capability). In addition, a cognitive system can be programmed to transmit and receive on a variety of frequencies and use different access technologies that are supported by its hardware design (reconfigurability) [8].

In order to provide cognitive capabilities, cognitive systems require novel radio frequency $(\mathrm{RF})$ transceiver architectures. The $\mathrm{CR}$ functionality mainly relies on the $\mathrm{RF}$ hardware technologies, such as wideband antenna, power amplifier, and adaptive filter. 
Therefore, the RF hardware should be capable of being tuned to any part of a large range of spectrum. In addition, a CR transceiver should be able to receive signals from various transmitters operating at different power levels, bandwidths, and locations [3].

The dynamic spectrum access (DSA) concept is not a novel idea, and it might be as old as radio communication itself. For instance, maritime communication systems were using shared radio resources in 1910s. The use of shared channels in land mobile communication were allowed by the FCC around 1960. In the 1970s, the Aloha protocol enabled sharing of radio channels without using a centralized entity. The integrated communications navigation and identification architecture (ICNIA) by Air Force Rome Labs was the first project to realize DSA in 1987. The ultra wideband communication was issued by the FCC 98-153 docket in 2002. Finally, CR has been employed to develop an air interface to opportunistically access the TV bands in regional area networks in 2011 [9]. Table 2.1 depicts a brief history for the CR-like systems mentioned above.

Table 2.1: A brief history of CR-like communication systems.

\begin{tabular}{|l|l|l|}
\hline Date & Project & Communication Scheme \\
\hline \hline 1910 & Maritime Communication & Hardware Radio \\
\hline 1960 & Shared Channel in Land Mobile Communications & Hardware Radio \\
\hline 1970 & ALOHA & Hardware Radio \\
\hline 1987 & ICNIA & Software Defined Radio \\
\hline 2002 & Ultra Wideband Communication & DSA \\
\hline 2011 & IEEE 802.22 & Cognitive Radio \\
\hline
\end{tabular}




\subsection{Cognitive Sensor Networks}

Rapid advances in processing capability, memory capacity, and radio technology have enabled the development of distributed networks with small and inexpensive communication nodes. These nodes are capable of sensing and communicating and can be deployed at a cost much lower than those of traditional wired sensor systems. Such systems are called wireless sensor networks (WSNs) $[10,11]$. The limited communication bandwidth assigned to WSNs is one of the main challenges of these networks.

To overcome the limited communication bandwidth assigned to WSNs, a new concept of cognitive sensor networks (CSNs) has been recently introduced in the literature [11-15]. A CSN is defined as a wireless network of low-power radios which gain secondary spectrum access following the CR paradigm [16]. Similar to the existing WSNs, a CSN consists of many tiny and inexpensive sensor nodes that operate on limited battery energy. Hence, sensor nodes are supposed to be energy efficient. In a WSN, each node either sends data, receives data, or it is in the idle state. However, in a CSN, there exists another state called spectrum sensing, where sensor nodes sense the spectrum to detect spectrum opportunities. Figure 2.1 depicts different states for these networks.

Adding cognition to WSNs provides several advantages. A CSN could provide access not only to new spectrum (rather than the worldwide available $2.4 \mathrm{GHz}$ band), but also to the spectrum with possibly better propagation characteristics [13]. For instance, 

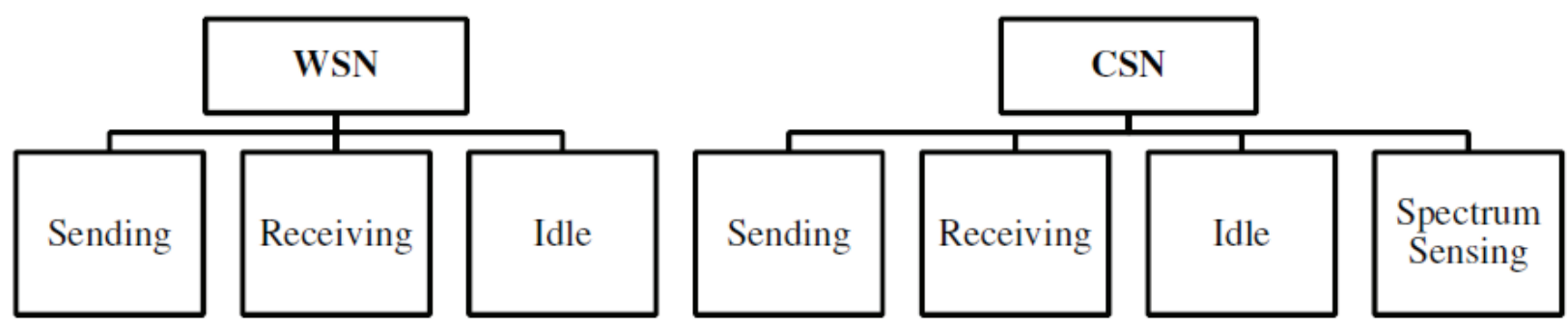

Figure 2.1: Different states in a WSN vs. a CSN.

the transmission range can be increased by using lower frequencies since the path loss

decreases as the operating frequency decreases [17]. The higher communication range

provides CSNs with a smaller number of hops per route. Thus, the average end-to-end

delays would also be smaller.

Due to the fact that sensor nodes are equipped with limited energy sources, researchers have been working on innovative methods to improve the network's energy consumption in CSNs. The sensing task is a repetitive process and it consumes extra energy from battery powered sensors. Therefore, it is important to employ energy-aware methods in these networks.

\subsection{Spectrum Sensing}

A CR network is designed to be aware of the changes in its surroundings. Spectrum sensing, therefore, is an important requirement for the realization of cognitive technology. Through spectrum sensing, cognitive users reliably detect the spectrum holes and protect 
PUs from the adverse interference from SUs. For instance, in IEEE 802.22 standard, spectrum sensing is included as a mandatory task, where SUs sense the spectrum for three different PUs: analog television, digital television, and licensed low-power auxiliary devices such as wireless microphones.

A variety of methods can be employed for this purpose. The existing sensing methods are classified into two main categories, as shown in Figure 2.2. In the first group of methods, the PU is in charge to provide SUs with the latest spectrum usage information. There are three schemes in this category as follows [18]: In the database registry method, the channel information is registered at a centralized database. In another method called beacon signals, the channel information is broadcasted on a predefined channel. In a third method, PUs periodically transmit a pilot signal on a subcarrier if that subcarrier is occupied by licensed users. By detecting the presence of such a pilot signal, SUs determine if that particular subcarrier is available or not. While leading to simplified secondary transceivers, these methods require some modifications to the current PUs and are incompatible with the existing primary network.

In the second group of methods, spectrum sensing solely relies on the secondary system to periodically sense the channel and detect the spectrum holes. In addition, SUs should monitor the activation of PUs to immediately vacate the occupied spectrum in case a PU appears. Due to its relatively low infrastructure cost and compatibility with 


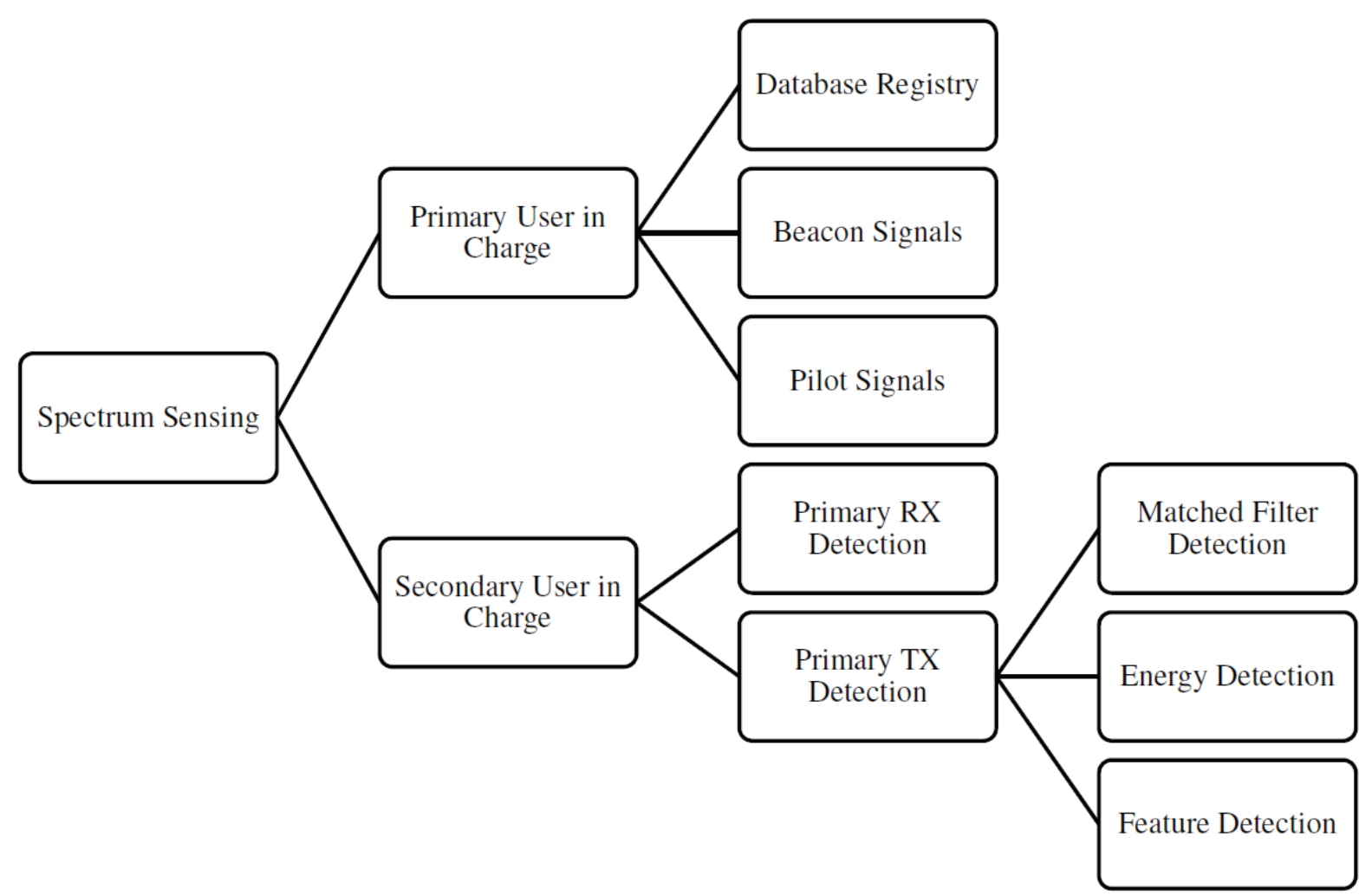

Figure 2.2: Classification of spectrum sensing methods.

the existing primary systems, the second group of methods has received more attention than other candidates. For instance, it is included in the IEEE 802.22 standard [19].

As shown in Fig. 2.2, there exist two schemes when SUs are in control: in the primary receiver detection method, secondary users detect spectrum holes by monitoring the primary users that are receiving data within their communication range. In reality, however, it is difficult for an SU to have a direct measurement of a channel between a primary receiver and a transmitter [17]. Thus, the most recent work focuses on the primary transmitter detection based on cognitive users' observation. The transmitter 
detection approach is based on the detection of weak signals from the PU through local observations of SUs. Three schemes are generally used for the transmitter detection method as follows $[17,20]$.

When the information of the PU's signal is known to the secondary network, the optimal detector in additive white Gaussian noise (AWGN) is the matched filter detector because it maximizes the received signal-to-noise ratio (SNR) [20]. However, a matched filter detector requires a priori knowledge of the primary signal such as the modulation type and the pulse shape. To this effect, if this information is not accurate, the method performs poorly.

If the receiver cannot gather sufficient information about the primary signal, a simpler alternative method called energy detection is helpful. An energy detector simply measures the energy received on a primary band during an observation time and declares a spectrum hole if the measured energy is less than a threshold. While compared to matched filtering, energy detection requires a longer sensing time to achieve a desired performance level, its low cost and implementation simplicity have made it a favorable candidate for spectrum sensing in CR.

A basic hypothesis model for the energy detection method can be defined as follows [21]: 


$$
r_{i}(t)= \begin{cases}n_{i}(t) & : H_{0} \\ k_{i}(t) s(t)+n_{i}(t) & : H_{1}\end{cases}
$$

where $r_{i}(t)$ is the received signal at the $i^{t h}$ secondary user, $s(t)$ is the transmitted signal by the PU, $n_{i}(t)$ is the AWGN at the $i^{t h} \mathrm{SU}$, and $k_{i}(t)$ is the channel gain of the sensing channel between the PU and the $i^{\text {th }} \mathrm{SU}$. The $H_{0}$ is a null hypothesis, which states that there is no primary signal in a certain spectrum band. On the other hand, $H_{1}$ is an alternative hypothesis, which indicates that there exists a primary signal.

Modulated signals are in general coupled with sine wave carriers. This characteristic has been used in the feature detection method, where the frequency characteristics are detected by analyzing a spectral correlation function. The main advantage of the feature detection method is that it can differentiate the noise energy from modulated signal energy, which is a result of the fact that the noise is a wide-sense stationary signal with no correlation to the primary signal. Therefore, a feature detector can perform better than energy detector in discriminating against noise due to its robustness to the uncertainty in noise power [22]. However, it is computationally complex and requires significantly long observation time [3, 23].

There are a number of difficulties in the noted methods. First, an SU may interfere 
with a PU in case the primary transmitter is not in the secondary user's communication range. As shown in Fig. 2.3 (a), the primary transmitter is not in the communication range of $S U_{1}$. Therefore, it starts to operate in the primary channel and interfere with $P U_{1}$. This is called the receiver uncertainty problem. Another difficulty arises in case an SU cannot detect a primary transmitter because of shadowing, and starts to communicate with a busy primary receiver. As shown in Fig. 2.3 (b), $S U_{1}$ cannot detect the primary transmitter due to shadowing from an obstacle, and thus interfere with $P U_{1}$. To overcome these problems, cooperative spectrum sensing schemes have been proposed in the literature $[24,25]$. A cooperative detection refers to the spectrum sensing methods where information from multiple SUs are incorporated to detect the presence of the primary network.

\subsection{Recent Advancements}

During the past few years, several major developments have been performed in the spectrum allocation policy to accelerate the opportunistic use of the radio spectrum. This includes the publication of the National Broadband Plan (NBP), a policy document released by the FCC that describes the spectrum management scheme to facilitate the broadband usage for the upcoming years. The NBP recommends providing further proceedings on opportunistic use of the spectrum in addition to the already completed TV 


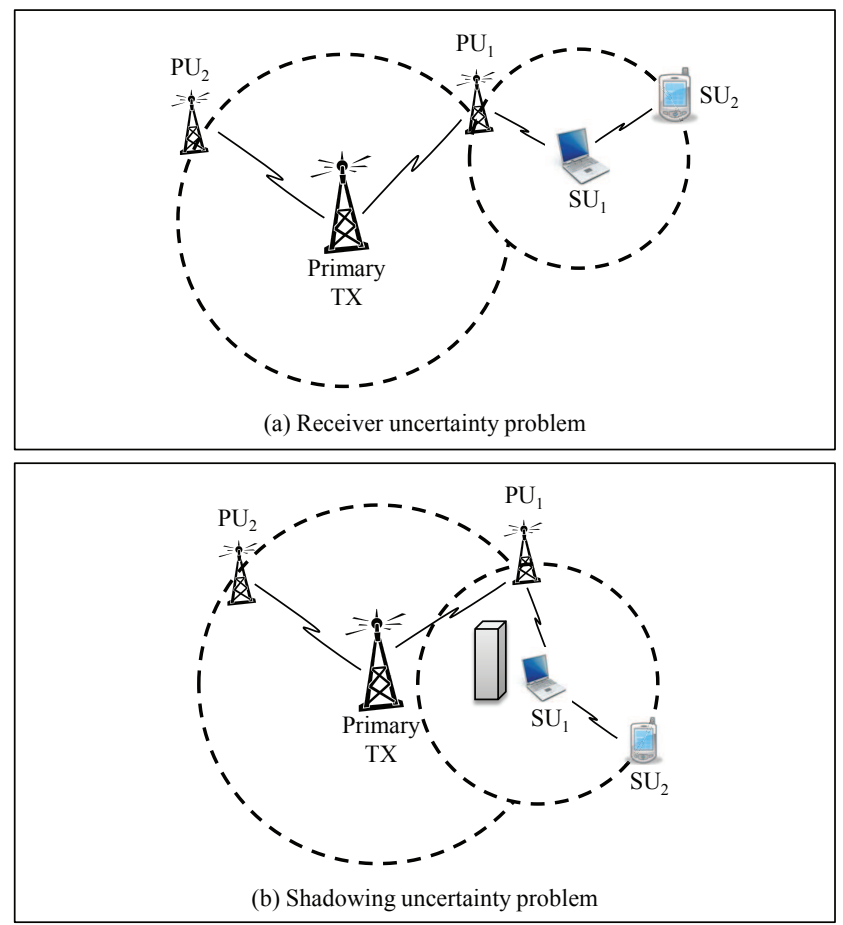

Figure 2.3: Receiver uncertainty and shadowing uncertainty problems in the transmitter detection method.

white spaces (TVWS) proceedings. Moreover, the plan recommends vacating $500 \mathrm{MHz}$ for broadband use within the next ten years, and $300 \mathrm{MHz}$ for mobile use in the next five years.

In addition, the IEEE 802.11 has standardized the final rules for unlicensed devices in TV bands [26]. A self-organizing network of beacon devices ${ }^{1}$ is under development (IEEE 802.22.1), which is expected to provide additional protection for low-power PUs. Moreover, the development of an IEEE 802.11 amendment to utilize the TVWS operation for WLANs is led by IEEE 802.11af. Also, the IEEE 802.19.1 Working Group is aiming

\footnotetext{
${ }^{1} \mathrm{~A}$ beacon device generates beacons so that the network users can detect its presence [27].
} 
to develop a standard for wireless coexistence in the TVWS that will help mitigating interference issues among CR-based Field Area Network/Automated Meter Infrastructure in smart grids. In addition, there is an ongoing effort for secondary use of the 2360-2400 MHz band for medical body area networks [28]. An IEEE 802.15 Study Group has been also created to investigate the use of TVWS.

In the field of sensor networks, which is mainly discussed in this dissertation, several research works have been directed towards applying cognitive technology in wireless sensor networks (WSNs) [13, 29-31]. The authors in [13] propose a CR-based WSN and compare its performance with a standard ZigBee/IEEE 802.15.4 WSN; both designs were built on the standard OPNET model that operate in the $2.4 \mathrm{GHz}$ band. By simulation work, the authors concluded that the maximum communication range in the CR-based WSN is almost twice the standard ZigBee/IEEE 802.15.4 WSN for the same transmission power. The longer communication range reduces the number of hops required in a multi-hop routing technique. In addition, it reduces the chance of having the receiver uncertainty problem. Therefore, it improves the efficiency of the multi-hop routing. Moreover, the authors claimed that the overall system throughput is improved in the CR-based WSN.

The main design principles, advantages, and applications of CSNs are discussed in [29]. In addition, the possibility of applying existing cognitive techniques to WSNs is 
investigated and several challenges in doing so are reported. Moreover, a distributed WSN-based control system for intelligent and reliable operation of large power grids is proposed in [30]. The network consists of several intelligent nodes that communicate with nearby nodes as well as a control station. The proposed method employs dynamic decision making and intelligent data fusion; therefore, even under changing environments, dynamic reconfiguration is possible without grid downtime. Also, a cognitive WSN that understands conflicting design objectives, finds optimum tradeoffs with given constraints, and adapts to the dynamics of the network is proposed in [31].

The energy efficiency has been also addressed as a key design metric in CSNs. For instance, a resource allocation problem from an energy efficiency standpoint is proposed in [32]. A fully distributed channel selection and power allocation scheme has been proposed to cover all subcarriers, subject to the required data rate and power constraints.

Recently, there have been worldwide efforts to provide international standards to utilize the spectrum through CR technology. The FCC in the United States, the Office of Communications (Ofcom) in the United Kingdom, and the Electronic Communications Committee (ECC) of the Conference of European Post and Telecommunications (CEPT) in Europe are the major regulatory agencies responsible for research and development of the opportunistic use of the spectrum. Several standards including IEEE 802.22 [26], IEEE 802.11af, ECMA 392 [33], IEEE SCC41, and ETSI RRS [34] are either under 
development or have already been finalized. In the following, we discuss a few standards in detail.

\section{IEEE 802.22}

The IEEE 802.22-based wireless regional area network (WRAN) aims to utilize the geographically unused spectrum by applying CR technology to coexist with the legacy TV services on 54-862 MHz band, and to provide broadband access to hard-to-reach lowpopulation-density areas such as rural environments [26]. The IEEE 802.22 standard, therefore, has been recognized as the CR standard [35]. Due to the fact that TV bands employ low frequency, their propagation characteristics are suitable for long-range transmission (up to 10-100 Km), and the IEEE 802.22 standard could be used for large-scale smart grid networks. Hence, the standard has the potential for wide applicability worldwide. While the IEEE 802.22 standard supports cognitive communication for WRANs, it is not intended to support low data rate, low energy consumption requirements of sensor networks. Currently, there is no standard for cognitive communication within CSNs.

In the IEEE 802.22 standard, spectrum sensing is listed as one of the main requirements of the cognitive process [36]. The sensing task consists of two stages: fast and fine sensing [37]. In the fast sensing stage, a coarse sensing algorithm is applied, such as the energy detection method. In the fine sensing stage, however, a more complicated 
method is employed such as waveform-based sensing, cyclostationary feature detection, or matched filtering method. The standard requires certain conditions to be met, such as the probability of detection has to be greater than 0.9 , and the probability of false alarm must be be smaller than 0.1 [38].

\section{ECMA 392}

ECMA 392 is the first CR standard that supports TVWS usage by personal/portable devices [39]. The standard was initiated by the Cognitive Networking Alliance (CogNeA), and a draft was later transferred to TC48-TG1. The standard documents the PHY and MAC layers with several characteristics including flexible network formation and real-time multimedia traffic support [39]. It is expected that new applications will be enabled by ECMA 392 such as in-home high-definition (HD) video streaming for personal/portable devices, campus-wide wireless coverage, and interactive TV broadcasting services.

\subsection{Summary}

This chapter discussed a brief introduction to the cognitive radio technology along with several definitions associated with the cognitive radio concept. The spectrum sensing was addressed as an important task in the realization of cognitive systems with an emphasize 
on the energy detection technique. The chapter also reviewed recent advancements and standards in the field of cognitive radio. 


\section{Chapter 3}

\section{Spectrum Occupancy Model}

\subsection{Background}

In CR, appropriate modeling of the spectrum occupancy by both primary and secondary networks is a prerequisite for CR analysis. The existence of a Markov chain by collecting real-time measurements in $928-948 \mathrm{MHz}$ is validated in [40]; the same methodology can be applied to any spectrum band. A CTMC model is setup for dynamic spectrum access in [41], where the radio systems attempt to operate in the same band. In [42], the wireless channel in a CR network is modeled by using a Hidden Markov Model (HMM) trained by the Genetic Algorithm. An HMM based channel status predictor is proposed in [43] which provides the CR network with the functionality to find alternative channels to perform the spectrum handover. Moreover, [44] assumes the spectrum usage statistics to 
be Poisson distributed, and proposes a technique to improve the performance of cognitive networks by performing predictions on the channel usage behavior of PUs.

A channel usage model for the PU usage pattern is proposed in $[45,46]$. The primary channel is modeled as an ON-OFF source alternating between ON (busy) and OFF (idle) periods. Once an OFF period is detected, SUs can utilize any portion of the remaining OFF period for their own transmission. The time until the next transition (sojourn time) of the ON and OFF periods for channel $i$ are modeled by random variables $T_{O N}^{i}$ and $T_{O F F}^{i}$ with the probability density function $(\mathrm{PDF}) f_{T_{O N}^{i}}(y), y>0$ and $f_{T_{O F F}^{i}}(x), x>0$ respectively. The $\mathrm{ON}$ and $\mathrm{OFF}$ periods are assumed to be independent and identically distributed (i.i.d.) random variables.

A CTMC approximation to model the primary channel usage pattern is suggested in $[47,48]$. Figure 3.1 depicts a transition diagram consisting of packet transmissions and idle periods, where $\lambda$ is the arrival rate of the $\mathrm{PU}$ and $\mu$ is the departure rate of the PU. The holding time in the transmit state corresponds to the length of the packets, which is largely influenced by the type of traffic. The packet lengths are assumed to be deterministic in the traffic scenario to be analyzed [49].

In [50], a CR network is studied with fixed primary network structure and mobile SUs in the same geographical area. As shown in Fig. 3.2, the primary network is further divided in several cells, where each cell consists of a single access point and several 


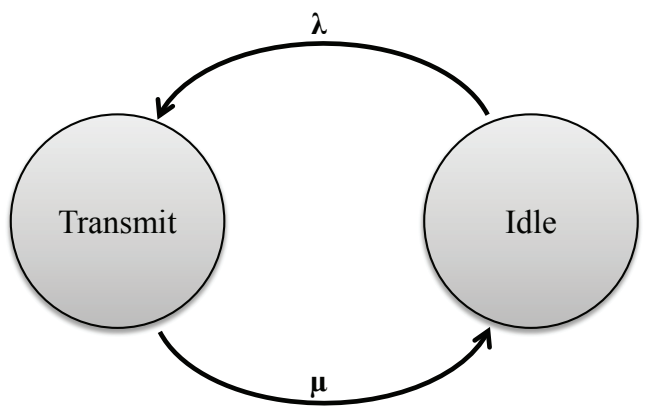

Figure 3.1: A CTMC approximation to model the primary channel usage pattern.

primary receivers. A mobile SU can only operate in the spectrum when its distance from the PU is greater than a system threshold or the PU is inactive, so as to avoid interference with licensed users. A three-state CTMC system is then developed that models the mobility-aware channel availability experienced by the mobile SUs. As shown in Fig. 3.3, the system can be at busy, idle or primary protection region (PPR) state. A primary channel is available when the $\mathrm{SU}$ is located outside the PPR, or the $\mathrm{SU}$ is located inside the PPR but the PU is idle. Next, the model is reduced into a two-state CTMC by merging the idle and PPR states into the OFF state, as shown in Fig. 3.3.

The process of spectrum occupancy as a CTMC system is modeled in [2]. The spectrum is assumed to consist of $M_{B}$ primary bands where each band is divided into $N_{S B}$ sub-bands. The $M_{B} \times N_{S B}$ sub-bands are shared by the primary and secondary networks. In this case, states are described by an integer pair $(i, j)$, where $i$ is the total number of sub-bands used by SUs and $j$ is the total number of primary bands used by PUs. The arrivals of SUs and PUs are assumed to be Poisson processes with arrival rates $\lambda_{a}$ and 


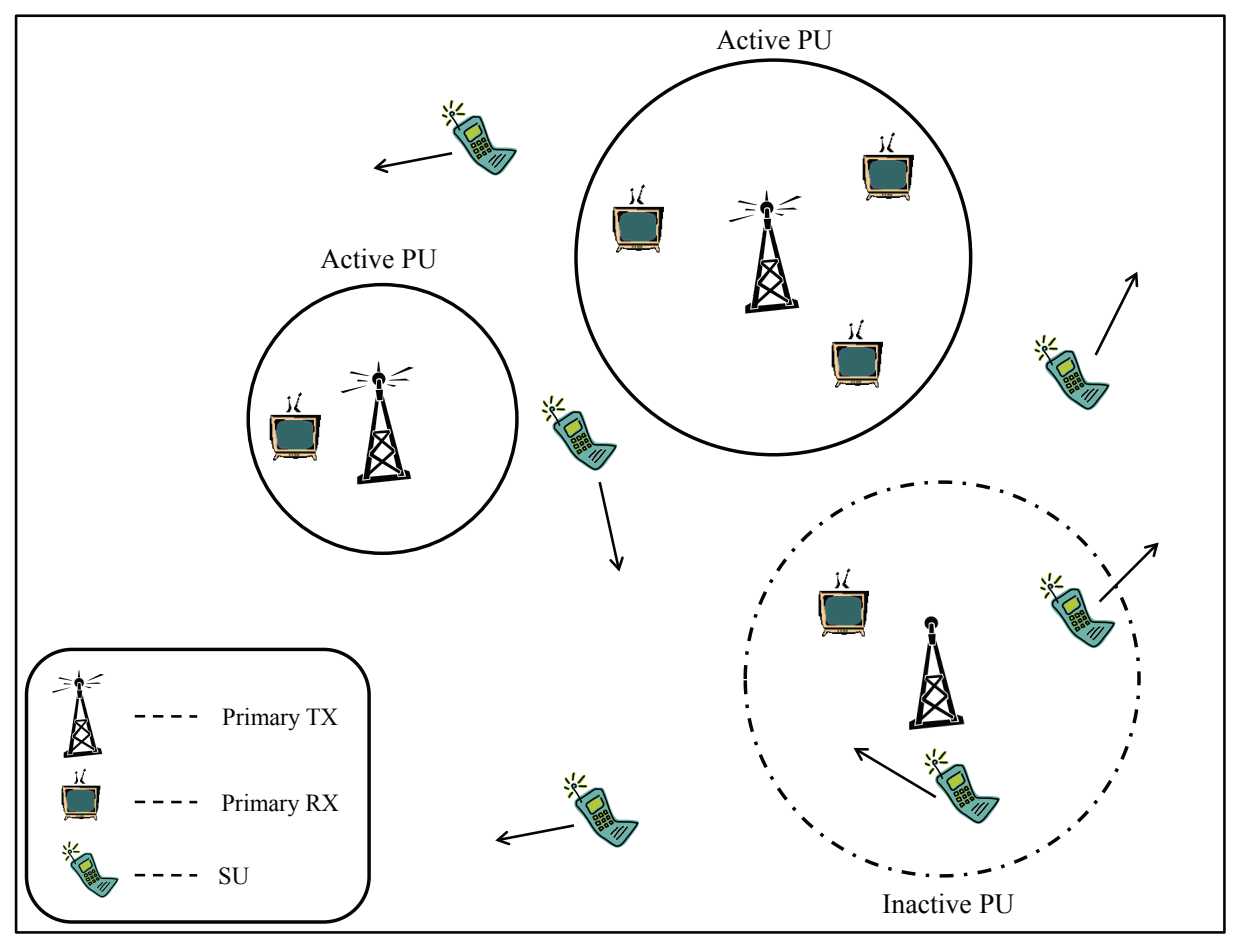

Figure 3.2: A CR network with mobile SUs.

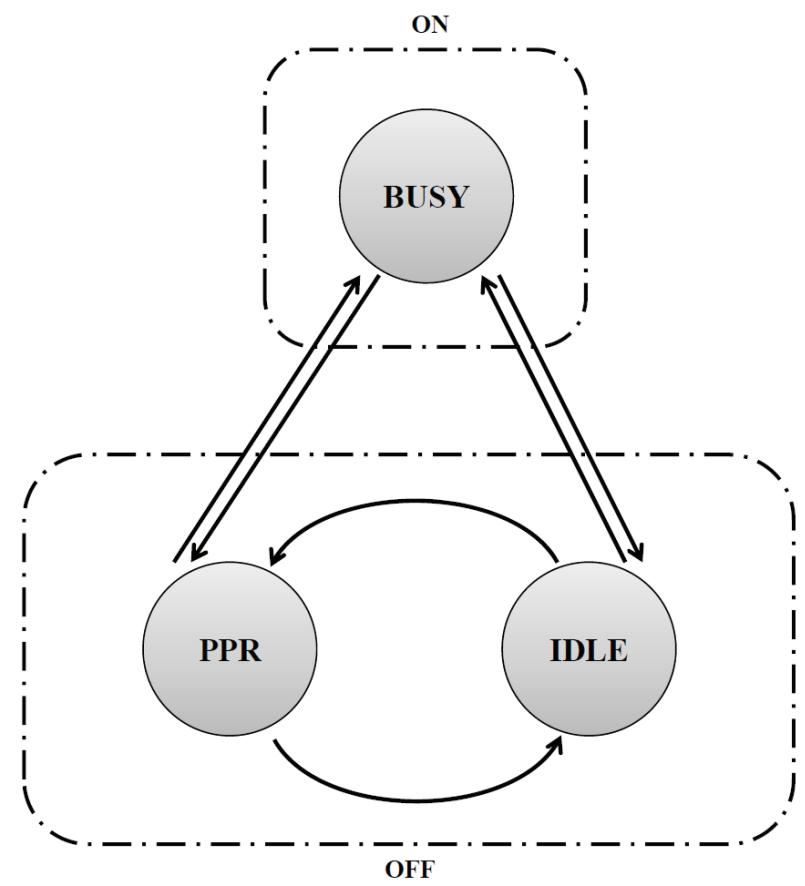

Figure 3.3: A three-state CTMC system to model the mobility-aware channel availability experienced by SUs. 
$\lambda_{b}$ respectively. The corresponding service times are also assumed to be exponentially distributed with rates $\mu_{a}$ and $\mu_{b}$ respectively.

As the PUs have the priority to use the spectrum, the SUs are forced to vacate the band if a PU operates in the spectrum. Depending on the number of sub-bands occupied by SUs in the new primary band, a forced termination in state $(i, j)$ will move the state to one of $(i, j+1),(i-1, j+1),(i-2, j+1), \ldots,\left(i-\left(N_{S B}-1\right), j+1\right),\left(i-N_{S B}, j+1\right)$ states, as shown in Fig. 3.4.

The majority of existing work assumes a simple cognitive system and analyze it using Markov modeling. A cognitive network with one primary and two secondary users is considered, and a primary-prioritized CTMC model is proposed in [51]. In [52], a CTMC model is proposed to predict the behavior of open (un-utilized) spectrum access in unlicensed bands. The model considers two types of secondary systems, but the analysis does not include the PU. Moreover, [2] presents the CTMC model for a simple case of one primary system and one secondary system.

In this chapter, we assume a CR network consisting of $N$ secondary users (with various traffic conditions) and one primary user $^{1}$. We describe the radio spectrum occupancy as a CTMC model, and analytically derive the transition rate matrix based on the model.

\footnotetext{
${ }^{1}$ This model is easily expanded in case of multiple primary users. As long as this model is considered, when a primary user starts transmission, the secondary users must quit transmission. Furthermore, the primary users share the channel with a predefined medium access control protocol.
} 


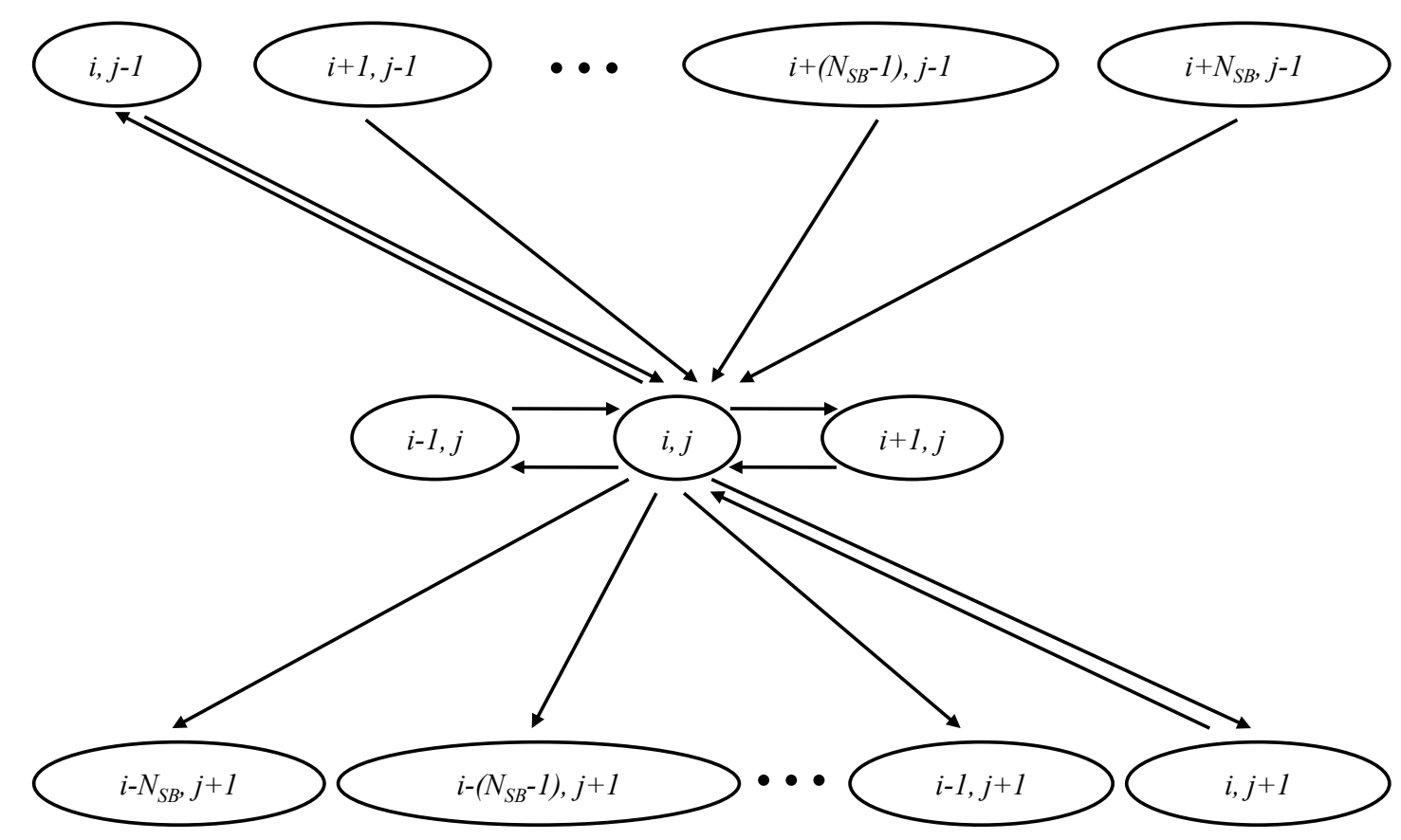

Figure 3.4: A Markov chain model for CR; $i$ is the total number of sub-bands used by SUs, and $j$ is the total number of primary bands used by the PU [2].

Moreover, an steady-state analysis is performed to derive the stationary state probability (SSP) vector.

\subsection{System Model}

A CR network with one primary user and $N$ secondary users $U=\left\{u_{i}: 1 \leq i \leq N\right\}$

is studied ${ }^{2}$. The secondary users are assumed to form a single-hop CR Network within

the transmission range of which there are no other secondary networks interfering or

\footnotetext{
${ }^{2}$ From the secondary network's perspective, the licensed channel cannot be used if a PU is present. In other words, it is not a secondary network's concern which PU is operating in the spectrum. We assume a representative for the PUs; therefore, the secondary network cannot use the spectrum if a primary representative is present.
} 
cooperating with that secondary network. Secondary users sense the primary channel to discover spectrum opportunities. Each secondary user is assumed to be equipped with a single identical antenna. We assume that the arrival and departure of the primary and secondary users' traffic are independent, continuous-time Poisson processes. Therefore, we can model the spectrum access process by a continuous-time Markov chain (CTMC) system. The primary users' traffic is modeled with two random processes. The service request is modeled as a Poisson process with arrival rate $\lambda_{P}\left(\mathrm{~s}^{-1}\right)$, and the service duration (access duration) is negative-exponentially distributed with mean time $1 / \mu_{P}(\mathrm{~s})$, so the departure of the primary user's traffic is another Poisson process with departure rate $\mu_{P}$ $\left(\mathrm{s}^{-1}\right)$ [51]. Similarly, the arrival and departure rates of secondary user $i(1 \leq i \leq N)$ are modeled as independent Poisson processes with $\lambda_{i}\left(\mathrm{~s}^{-1}\right)$ and $\mu_{i}\left(\mathrm{~s}^{-1}\right)$ respectively.

We assume that the spectrum cannot be occupied by more than one user at any time, i.e., there is no overlap between any two users. Therefore, the spectrum access process is given by the model shown in Fig. 3.5. The state space vector $\mathbf{x}$ for the model is

$$
\mathbf{x}=\left\{O, S_{1}, S_{2}, \ldots, S_{N}, P\right\}
$$

In (3.1), state $O$ (idle) means no user operates in the spectrum, state $P$ means the primary user operates in the spectrum, and state $S_{i}$ means the $i^{\text {th }}$ secondary user operates 
in the spectrum $(1 \leq i \leq N)$. We assume the initial state of the system to be state $O$. As shown in Fig. 3.5, the system enters state $P$ with arrival rate $\lambda_{P}$ whenever the primary user appears, and it returns to state $O$ with departure rate $\mu_{P}$ when the primary user's service is complete. While the system is in state $O$, and the $i^{\text {th }}$ secondary user enters into the spectrum, the system enters state $S_{i}$ with arrival rate $\lambda_{i}$. In case the primary user arrives before the secondary user $i$ 's service is complete, the system transits to state $P$ with arrival rate $\lambda_{P}$. Otherwise, the $i^{\text {th }}$ secondary user returns to state $O$ with departure rate $\mu_{i}$ when its service is complete. In the CTMC, when the secondary users contend to access the idle spectrum using carrier sense multiple access, collisions occur only when their service requests arrive exactly at the same time; this case rarely happens for independent Poisson process [51]. Therefore, we disregard the collision state of the secondary users.

\subsection{Derivation of the Steady-State Probabilities}

The state space vector $(\mathbf{x})$ and the transition rate matrix $\mathbf{Q}$ are needed for a complete CTMC model definition [53]. The state space vector is defined in (3.1). The transition 


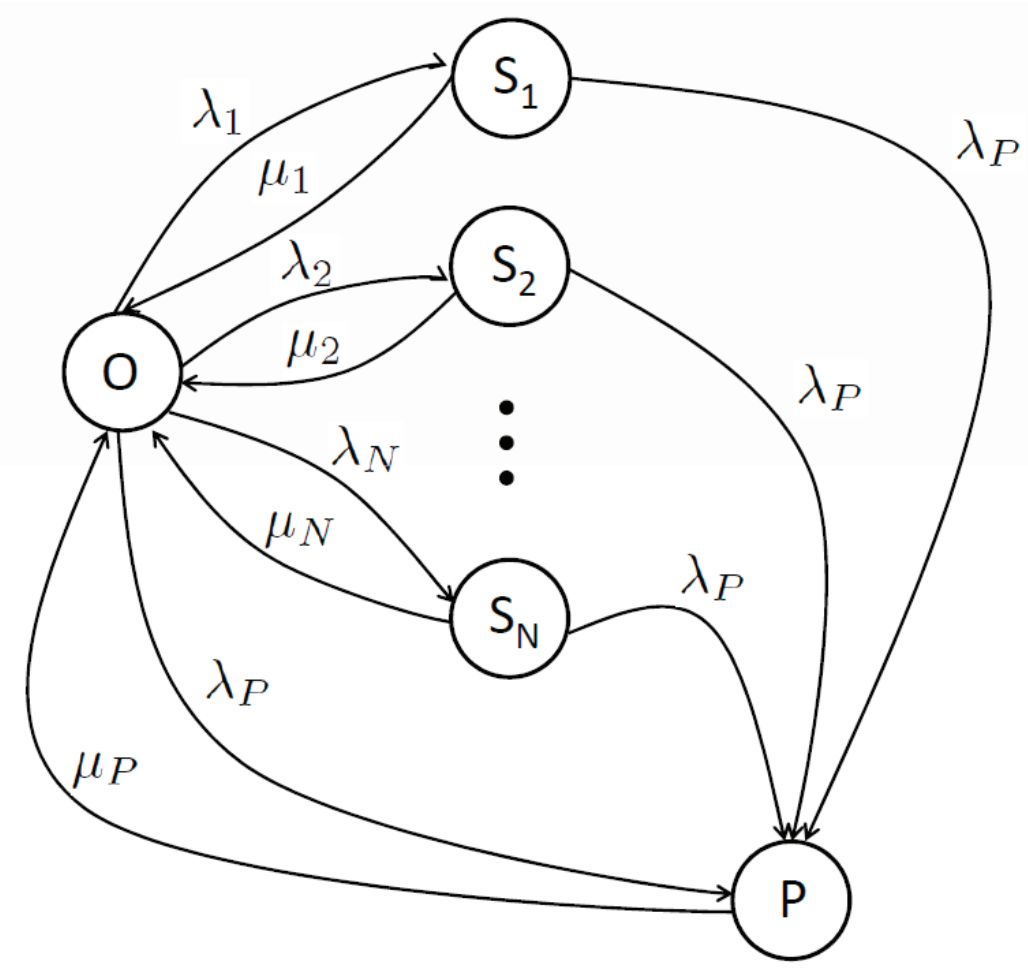

Figure 3.5: The state transition rate diagram for the proposed CTMC model.

rate matrix is obtained for the CTMC model shown in for Fig. 3.5, as follows:

$\mathbf{Q}=\left[\begin{array}{cccccc}-\left(\lambda_{1}+\lambda_{2}+\ldots+\lambda_{N}+\lambda_{P}\right) & \lambda_{1} & \lambda_{2} & \ldots & \lambda_{N} & \lambda_{P} \\ \mu_{1} & -\left(\mu_{1}+\lambda_{P}\right) & 0 & \ldots & 0 & \lambda_{P} \\ \mu_{2} & 0 & -\left(\mu_{2}+\lambda_{P}\right) & \ldots & 0 & \lambda_{P} \\ \vdots & \vdots & \vdots & \ddots & \vdots & \vdots \\ \mu_{N} & 0 & 0 & \ldots & -\left(\mu_{N}+\lambda_{P}\right) & \lambda_{P} \\ \mu_{P} & 0 & 0 & \ldots & 0 & -\mu_{P}\end{array}\right]$

In the derivation of (3.2), the following points have been taken into consideration: 
- It is assumed that the system is stationary where all the transition probabilities, $P_{i j}(s, t)=P[x(t)=j \mid x(s)=i],(s \leq t$, and $i, j \in \mathbf{x})$, are independent of the absolute time instants $s$ and $t$, and depend on only the time difference $(t-s)$ [54]. Therefore, the transition rate matrix $\mathbf{Q}(t)$ is independent of $t$, that is, $\mathbf{Q}(t)=\mathbf{Q}$.

- An off-diagonal element of the transition rate matrix, $\left(q_{i j},(i \neq j)\right)$, represents the Poisson process rate of transition from state $i$ to state $j$.

- A diagonal element $q_{i i},(1 \leq i \leq N+2)$ is determined by

$$
q_{i i}=-\sum_{a l l j, i \neq j} q_{i j}
$$

where $1 \leq j \leq N+2$.

An important objective of Markov chain analysis is to determine the state probability vector $\Pi(t)=\left[\Pi_{0}(t), \Pi_{1}(t), \Pi_{2}(t), \ldots, \Pi_{N}(t), \Pi_{P}(t)\right]$, where the $i^{t h}$ element of $\Pi(t)$ is given by [55]

$$
\Pi_{i}(t)=P[x(t)=i], \forall i \in \mathbf{x}
$$

The $\Pi_{i}(t)$ denotes the probability to find the system at state $i$ at specific time instants. Now, we perform the steady-state analysis by assuming that system has been running long enough, and all parameters have achieved their steady state values [56]. Therefore 
the steady-state probability of state $i$ is

$$
\Pi_{i}=\lim _{t \rightarrow \infty} \Pi_{i}(t)
$$

The steady-state probability (SSP) vector ( $\boldsymbol{\Pi}$ ) is determined by solving (3.6) and (3.7) given below:

$$
\begin{gathered}
\Pi \mathbf{Q}=\mathbf{0} \\
\sum_{\forall j \in \mathbf{x}} \Pi_{j}=1
\end{gathered}
$$

The matrix equation in (3.6) can be expanded into a system of linear equations presented in (3.8-3.10), as follows:

$$
\begin{gathered}
-\Pi_{0}\left(\lambda_{1}+\lambda_{2}+\ldots+\lambda_{N}+\lambda_{P}\right)+\Pi_{1} \mu_{1}+\Pi_{2} \mu_{2}+\ldots+\Pi_{N} \mu_{N}+\Pi_{P} \mu_{P}=0 \\
\Pi_{0} \lambda_{i}-\Pi_{i}\left(\mu_{i}+\lambda_{P}\right)=0 \quad(1 \leq i \leq N), \\
\left(\Pi_{0}+\Pi_{1}+\ldots+\Pi_{N}\right) \lambda_{P}-\Pi_{P} \mu_{P}=0 .
\end{gathered}
$$


Substituting $\Pi_{P}$ from (3.8) into (3.7), we get

$$
\begin{array}{r}
\Pi_{0}+\Pi_{1}+\ldots+\Pi_{N}+\Pi_{P}=1 \Rightarrow \\
\Pi_{0}+\Pi_{1}+\ldots+\Pi_{N}+\frac{\lambda_{P}}{\mu_{P}}\left(\Pi_{0}+\Pi_{1}+\ldots+\Pi_{N}\right)=1 \Rightarrow \\
\Pi_{0}+\Pi_{1}+\ldots+\Pi_{N}=\frac{\mu_{P}}{\mu_{P}+\lambda_{P}} .
\end{array}
$$

Now, we substitute (3.9) into (3.11) to obtain $\Pi_{0}$ :

$$
\begin{array}{r}
\Pi_{0}+\frac{\Pi_{0} \lambda_{1}}{\mu_{1}+\lambda_{P}}+\ldots+\frac{\Pi_{0} \lambda_{N}}{\mu_{N}+\lambda_{P}}=\frac{\mu_{P}}{\mu_{P}+\lambda_{P}} \Rightarrow \\
\Pi_{0}\left(1+\frac{\lambda_{1}}{\mu_{1}+\lambda_{P}}+\ldots+\frac{\lambda_{N}}{\mu_{N}+\lambda_{P}}\right)=\frac{\mu_{P}}{\mu_{P}+\lambda_{P}} \Rightarrow \\
\Pi_{0}=\frac{\mu_{P}}{\left(\mu_{P}+\lambda_{P}\right)\left(1+\sum_{i=1}^{N} \frac{\lambda_{i}}{\mu_{i}+\lambda_{P}}\right)} .
\end{array}
$$

Therefore, $\Pi_{1}, \Pi_{2}, \ldots, \Pi_{N}$ given by (3.9) are as follows:

$$
\Pi_{i}=\frac{\lambda_{i}}{\mu_{i}+\lambda_{P}} \Pi_{0} \quad(1 \leq i \leq N)
$$

where $\Pi_{0}$ is given by $(3.12)$.

Finally, we substitute (3.11) into (3.7) to derive $\Pi_{P}$ as follows:

$$
\Pi_{P}=1-\Pi_{0}-\Pi_{1}-\ldots-\Pi_{N}=1-\frac{\mu_{P}}{\mu_{P}+\lambda_{P}}=\frac{\lambda_{P}}{\mu_{P}+\lambda_{P}}
$$


Therefore, the SSP vector is determined by (3.12)-(3.14). As shown in (3.14), the probability of being in state $P$ is solely a function of the primary user parameters $\left(\lambda_{p}\right.$ and $\left.\mu_{p}\right)$ as expected. However, the probability of being in state $S_{i}\left(\Pi_{i}\right)$ is a function of both the primary and secondary user parameters as well as the number of secondary users $(N)$.

\subsection{Numerical Analysis}

In this section, we perform numerical analysis to evaluate our model. First, we study a CR network with no active secondary user $(N=0)$, and compare the proposed model with an existing model in the literature. Second, we consider a CR network with three secondary users $(N=3)$ and examine the effects of the secondary users' arrival and departure rates on the SSP vector. Finally, we examine a case with a very large number of secondary users.

\subsubsection{No Active Secondary User $(N=0)$}

Here, the proposed model is evaluated for a special case where $(N=0)$. Fig. 3.6(a) depicts the state transition diagram in this case, where the state space vector is 
$\mathbf{x}=\{O, P\}$. The SSP vector $\boldsymbol{\Pi}=\left[\Pi_{0}, \Pi_{P}\right]$ is given by (3.12) and (3.14) as follows:

$$
\begin{aligned}
& \Pi_{0}=\frac{\mu_{P}}{\mu_{P}+\lambda_{P}} \\
& \Pi_{P}=\frac{\lambda_{P}}{\mu_{P}+\lambda_{P}}
\end{aligned}
$$

In [1], the primary channel is modeled as an ON-OFF source alternating between ON (busy) and OFF (idle) periods, as shown in Fig. 3.6-(b). Two parameters $(u)$ and $(1-u)$ have been defined in [1] as the fraction of time in which the primary channel is in ON and OFF respectively, as follows:

$$
\begin{gathered}
u=\frac{\lambda_{O F F}}{\lambda_{O N}+\lambda_{O F F}} \\
1-u=\frac{\lambda_{O N}}{\lambda_{O N}+\lambda_{O F F}}
\end{gathered}
$$

where $\lambda_{O F F}$ and $\lambda_{O N}$ are the inverse of the mean values of $T_{O F F}$ and $T_{O N}$ respectively, i.e., $E\left[T_{O F F}\right]=\frac{1}{\lambda_{O F F}}$ and $E\left[T_{O N}\right]=\frac{1}{\lambda_{O N}}$.

As can be seen in (3.15-3.18), $\Pi_{P}$ and $\Pi_{0}$ in our model represent $u$ and $(1-u)$ in [1] respectively. Moreover, $\lambda_{O F F}$ and $\lambda_{O N}$ in [1] correspond to $\mu_{P}$ and $\lambda_{P}$ respectively. Therefore, our proposed model at this special case $(N=0)$ agrees with the model in [1]. Table 3.1 summarizes the above notations and results. 


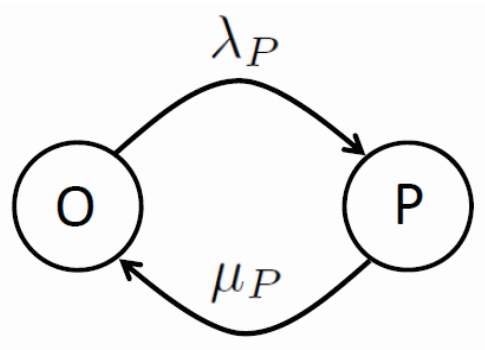

(a)

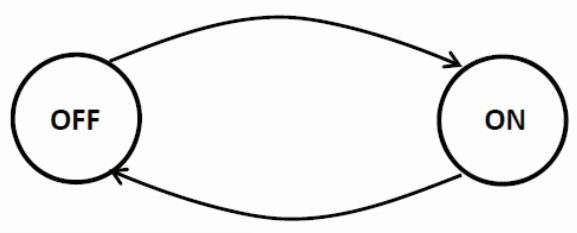

$$
T_{O F F} \sim f_{T_{O F F}}(x), x>0 \quad T_{O N} \sim f_{T_{O N}}(y), y>0
$$

(b)

Figure 3.6: The state transition diagram in: (a) our model at $N=0$, (b) the existing model in [1].

\subsubsection{Three Secondary Users $(N=3)$}

Here, a CR network with three secondary users $(N=3)$ is considered, where the state space vector is $\mathbf{x}=\left\{O, S_{1}, S_{2}, S_{3}, P\right\}$, and the state transition diagram is shown in Fig.

3.7. The secondary users' stationary state probabilities $\left(\Pi_{1}, \Pi_{2}, \Pi_{3}\right)$ are given by $(3.13)$, and $\Pi_{P}$ is calculated by (3.14). For this case, we arbitrarily set the primary user's arrival and departure rates to be $\lambda_{P}=2\left(\mathrm{~s}^{-1}\right)$ and $\mu_{P}=4\left(\mathrm{~s}^{-1}\right)$ respectively, and investigate the effect of secondary users' parameters on the SSP vector for three cases as follows:

Table 3.1: The proposed model at $N=0$ agrees with the existing model in [1].

\begin{tabular}{c|c}
\hline \hline The proposed model & Existing model $[1]$ \\
\hline$\mu_{P}$ & $\lambda_{O F F}$ \\
\hline$\lambda_{P}$ & $\lambda_{O N}$ \\
\hline$\Pi_{P}=\frac{\lambda_{P}}{\lambda_{P}+\mu_{P}}$ & $u=\frac{\lambda_{O F F}}{\lambda_{O N}+\lambda_{O F F}}$ \\
\hline$\Pi_{0}=\frac{\mu_{P}}{\lambda_{P}+\mu_{P}}$ & $1-u=\frac{\lambda_{O N}}{\lambda_{O N}+\lambda_{O F F}}$ \\
\hline
\end{tabular}




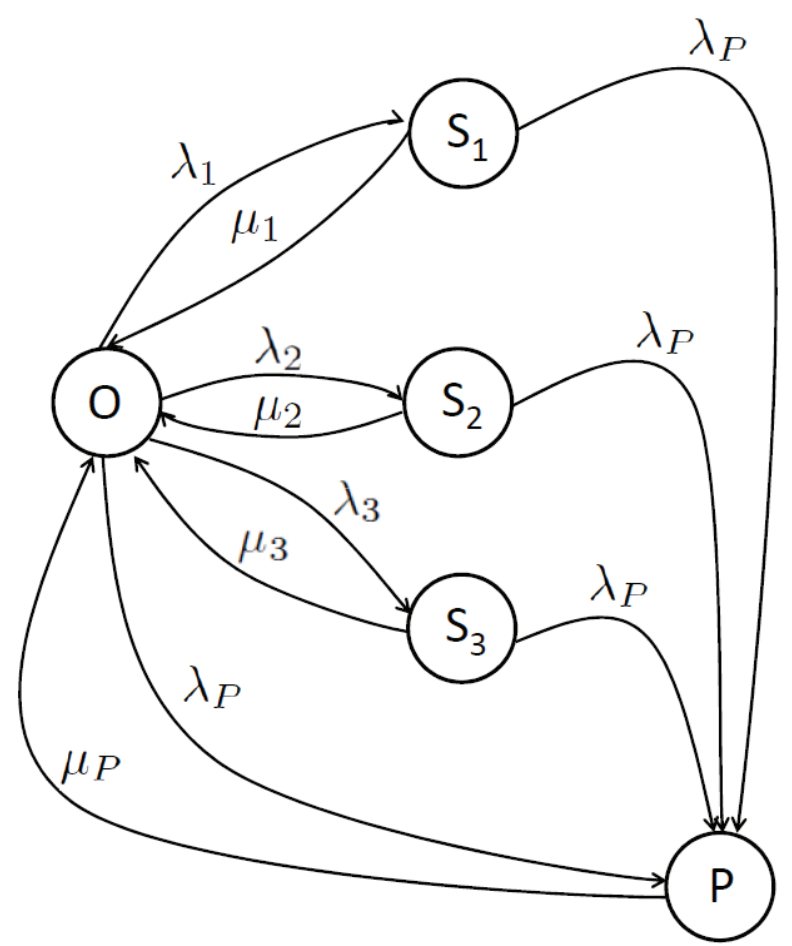

Figure 3.7: The state transition rate diagram for $N=3$.

\section{Case-I: Different Departure Rates and Identical Arrival Rates}

We assume different departure rates $\mu_{1}<\mu_{2}<\mu_{3}$, and assume identical arrival rates $\lambda_{1}=\lambda_{2}=\lambda_{3}=\lambda$. Thus, (3.13) simplifies to

$$
\Pi_{i}=\frac{\lambda}{\mu_{i}+\lambda_{P}} \Pi_{0} \quad(1 \leq i \leq 3)
$$

From the above equation, $\mu_{1}<\mu_{2}<\mu_{3}$ yields $\Pi_{1}>\Pi_{2}>\Pi_{3}$, which confirms that for a fixed value of $\lambda$, there is a higher probability to find the system at a state with lower departure rate $(\mu)$. Fig. 3.8 (a) shows the stationary state probabilities for a secondary 
network with the following parameters, where the secondary user $i$ is denoted by $S U_{i}$, and $\lambda_{i}$ and $\mu_{i}$ are both in $\left(\mathrm{s}^{-1}\right)$.

$$
S U_{1}:\left\{\begin{array}{l}
\lambda_{1}=3 \\
\mu_{1}=2
\end{array} \quad, S U_{2}:\left\{\begin{array}{l}
\lambda_{2}=3 \\
\mu_{2}=4
\end{array} \quad, S U_{3}:\left\{\begin{array}{l}
\lambda_{3}=3 \\
\mu_{3}=6
\end{array}\right.\right.\right.
$$

The SSP vector in this case is $\Pi=[0.2540,0.1905,0.1270,0.0952,0.3333]$.

\section{Case-II: Different Arrival Rates and Identical Departure Rates}

We assume different arrival rates $\lambda_{1}<\lambda_{2}<\lambda_{3}$ but identical departure rates $\mu_{1}=\mu_{2}=$ $\mu_{3}=\mu$. Therefore, (3.13) simplifies to

$$
\Pi_{i}=\frac{\lambda_{i}}{\mu+\lambda_{P}} \Pi_{0} \quad(1 \leq i \leq 3) .
$$

As shown in (3.20), $\lambda_{1}<\lambda_{2}<\lambda_{3}$ yields $\Pi_{1}<\Pi_{2}<\Pi_{3}$. This confirms that for a fixed value of $\mu$, there is a higher probability to find the system at a state with larger arrival rate $(\lambda)$. The secondary users' parameters are set as follows:

$$
S U_{1}:\left\{\begin{array}{l}
\lambda_{1}=2 \\
\mu_{1}=3
\end{array} \quad, S U_{2}:\left\{\begin{array}{l}
\lambda_{2}=4 \\
\mu_{2}=3
\end{array} \quad, S U_{3}:\left\{\begin{array}{l}
\lambda_{3}=6 \\
\mu_{3}=3
\end{array}\right.\right.\right.
$$




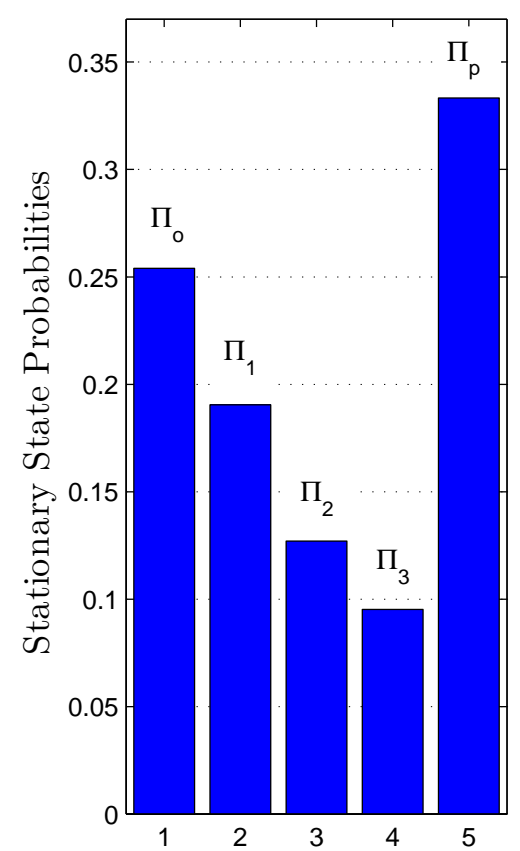

(a) Network Users, Case-I

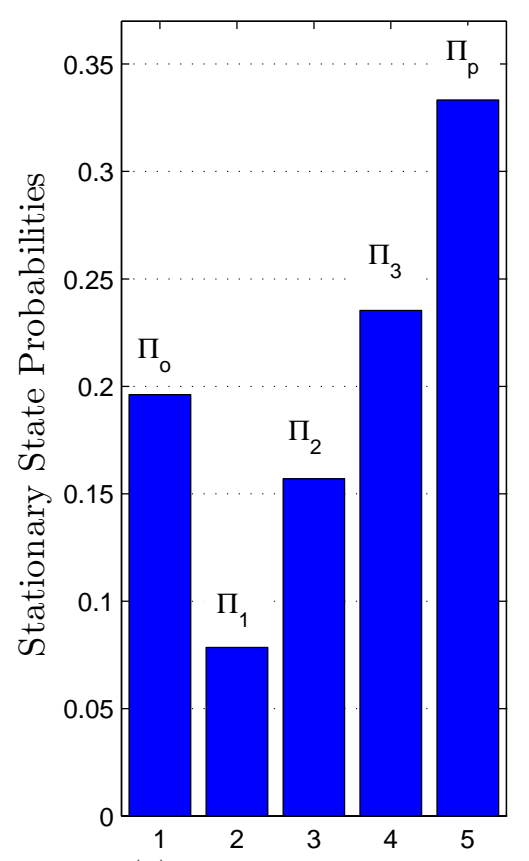

(b) Network Users, Case-II

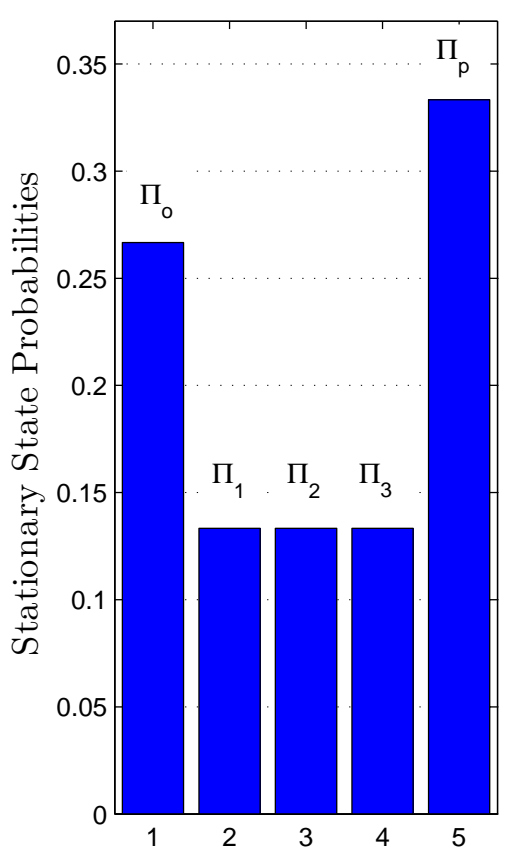

(c) Network Users, Case-III

Figure 3.8: The stationary state probabilities for cases (a), (b), and (c).

The SSP vector is $\Pi=[0.1961,0.0784,0.1569,0.2353,0.3333]$. Fig. 3.8 (b) shows the stationary state probabilities for this case.

\section{Case-III: Identical Departure and Arrival Rates}

In this part, we assume a secondary network where all secondary users have the same arrival rates $\left(\lambda_{1}=\lambda_{2}=\ldots=\lambda_{N}=\lambda\right)$ and also have the same departure rates $\left(\mu_{1}=\right.$ $\left.\mu_{2}=\ldots=\mu_{N}=\mu\right)$. First, the SSP vector for a general case with $N$ secondary users is derived. Then, we consider a secondary network with $N=3$ users, and sketch the stationary state probabilities similar to the previous cases. Therefore, all the secondary 
users have the same stationary state probabilities:

$$
\Pi_{i}=\frac{\lambda \mu_{P}}{\left(\mu_{P}+\lambda_{P}\right)\left(\mu+\lambda_{P}+N \lambda\right)} \quad(1 \leq i \leq N)
$$

Thus, for a CR network with $N=3$ secondary users, and secondary users' parameters $\lambda=4 \mathrm{~s}^{-1}$ and $\mu=6 \mathrm{~s}^{-1}$, the SSP vector is $\boldsymbol{\Pi}=[0.2667,0.1333,0.1333,0.1333,0.3333]$. Fig. 3.8 (c) shows the stationary state probabilities for this case.

Note that the primary user's stationary state probability $\left(\Pi_{P}\right)$ presented in $(3.14)$ is not a function of the number of secondary users $(N)$ and the secondary users' parameters $\left(\lambda\right.$ and $\mu$ ). Therefore, in all the above cases, $\Pi_{P}$ has the same value $\Pi_{P}=0.3333$.

\subsubsection{Very Large Number of Secondary Users}

Here, we consider a CR network with a very large number of secondary users $(N=100)$.

Without loss of generality, we randomly select each secondary user's arrival and departure rates within $0.1 \leq \lambda_{i} \leq 5$ and $0.1 \leq \mu_{i} \leq 7.5$, and we set the primary user's parameters

to be $\lambda_{P}=2.5\left(\mathrm{~s}^{-1}\right)$ and $\mu_{P}=5\left(\mathrm{~s}^{-1}\right)$. Secondary users' stationary state probabilities are determined by (3.12) and (3.13), and $\Pi_{P}$ is given by (3.14).

In the CTMC model, the primary user is granted the highest priority to operate in the spectrum. Consequently, $\Pi_{P}$ is independent of the number of secondary users $(N)$, 


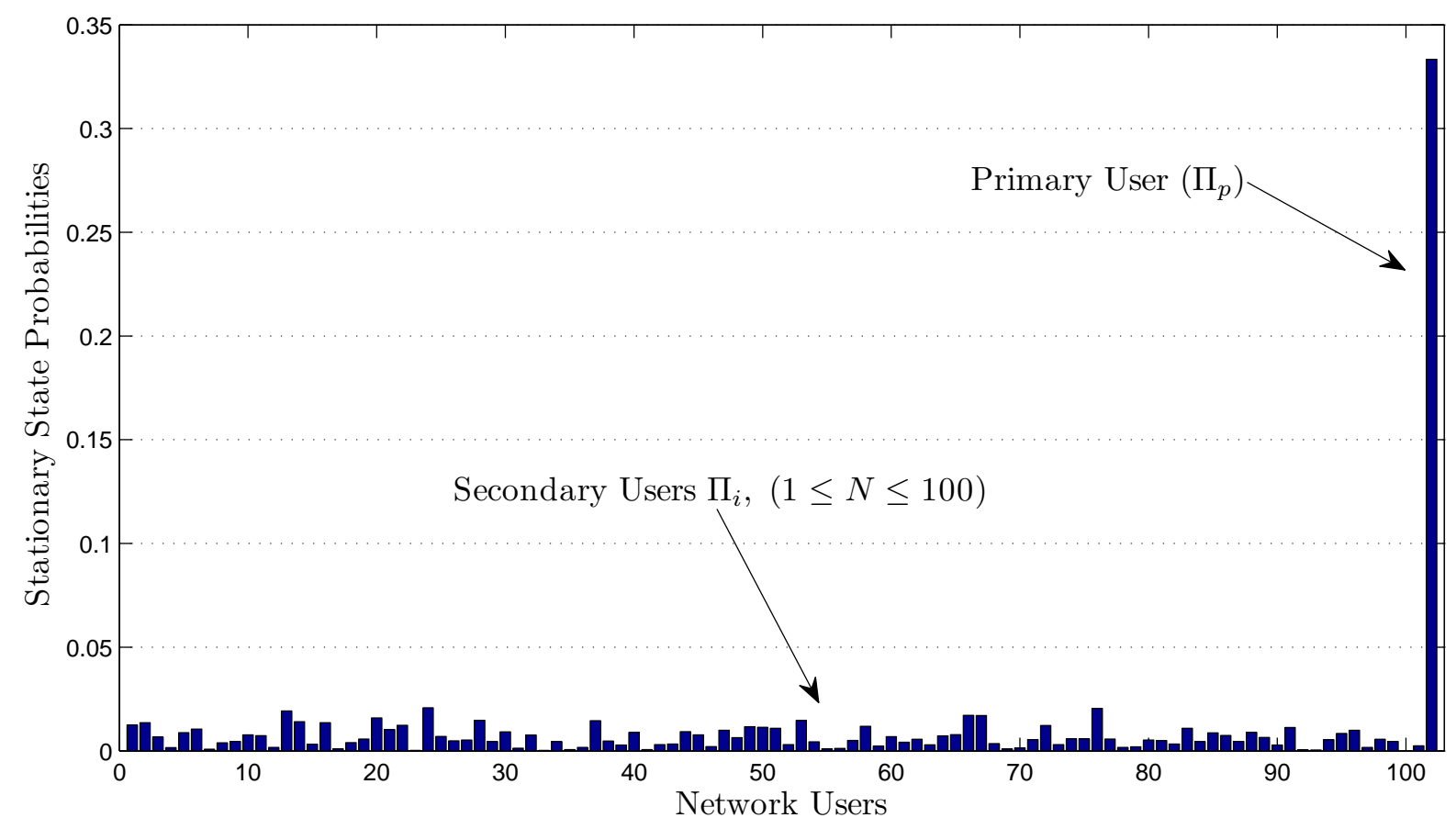

Figure 3.9: The stationary state probabilities in a secondary network with $N=100$ SUs.

and does not change with a large $N$. As a result, the rest of the spectrum (which has not been used by the primary user) would be available for the secondary access. As shown in Fig. 3.9, due to the very large number of secondary users in this case $(N=100)$, the probability to find the system at each secondary state is quite small.

\subsection{Summary}

The modeling of the spectrum occupancy in a CR network was studied in this chapter.

A continuous-time Markov chain (CTMC) was used to describe the spectrum occupation by both licensed and unlicensed users. By analytical work, we derived the transition rate 
matrix and the probability state vector for the proposed model. In addition, numerical analysis was conducted to examine the proposed model, and to compare the proposed model with an existing model in the literature. We will use the CTMC model as a base model for further analysis in the following chapters. 


\section{Chapter 4}

\section{Application-Specific Spectrum}

\section{Sensing}

\subsection{Motivations}

As the CR concept matures, CR networks are now envisioned to support a variety of applications, ranging from the smart grid, public safety and broadband cellular, and medical applications to wireless sensor networks (WSNs) [11, 57-59]. For instance, in the aerospace industry, major aircraft manufacturers and many supply research groups have shown strong interest in development and standardization of sensor networks for onboard use within aircraft, e.g., the "Wireless Avionics Intra-Communications (WAIC)" project by the Aerospace Vehicle System Institute. The WAIC refers to radio communication 
between two or more points within a single aircraft (not air-to-ground or air-to-air) [60]. Table 4.1 shows several examples of WAIC applications. As depicted, different applications have various design requirements; for instance, a sensor network for temperature monitoring requires a low data rate link while some other application may require a high data rate link. From another point of view, an engine prognostic sensor network may have a high probability of presence due to its large data arrival rate, while a tire pressure sensor network may have a low probability of presence due to its small data arrival rate.

While a CR network is supposed to change its parameters according to the characteristics of both primary and secondary network environments, most existing algorithms consider only the primary network's characteristics to obtain the optimum sensing period. This implies that the optimum sensing period would be identical for diverse CR applications, while their characteristics could be vastly different. For instance, Fig. 4.1 compares a non-application-specific sensing approach with an application-specific sensing scheme in CR. The PU's properties are assumed to be the same for the two types of the secondary applications. As shown, in a non-application-specific approach, the sensing

Table 4.1: A few examples of sensor networks in the WAIC project.

\begin{tabular}{|l||l|l|}
\hline Application Type & Low Data Rate & High Data Rate \\
\hline \hline Sensing & Fuel Tank, Temperature & $\begin{array}{l}\text { Engine Prognostic, Structural } \\
\text { Health Monitoring }\end{array}$ \\
\hline $\begin{array}{l}\text { Control and Com- } \\
\text { munication }\end{array}$ & Cabin Functions & Avionics Communications Bus \\
\hline
\end{tabular}


period is identical for both applications. On the other hand, in the application-specific sensing method, the sensing period is selected to be greater for an application that has a lower probability of presence. This results in significant improvement in the usage of the CR network's valuable resources such as time and energy.

The desired sensing method, therefore, must distinguish different types of secondary applications, and provide a customized solution for each type. In this chapter, we propose an application-specific spectrum sensing method that considers both secondary and primary users characteristics, and accurately finds the optimum sensing period. The proposed method aims to reduce the cognitive network's energy consumption by avoiding unnecessary sensing tasks, while meeting the sensing requirements. To the best of our knowledge, this is the first work showing that significant power consumption can be obtained by considering the properties of both primary and secondary networks in cognitive radio.

\subsection{Energy Detection Method}

Recently, a number of methods have been proposed for spectrum sensing, including energy detection, matched filter detection, and cyclostationary feature detection. Among these methods, energy detection is the most popular spectrum sensing technique due to its 


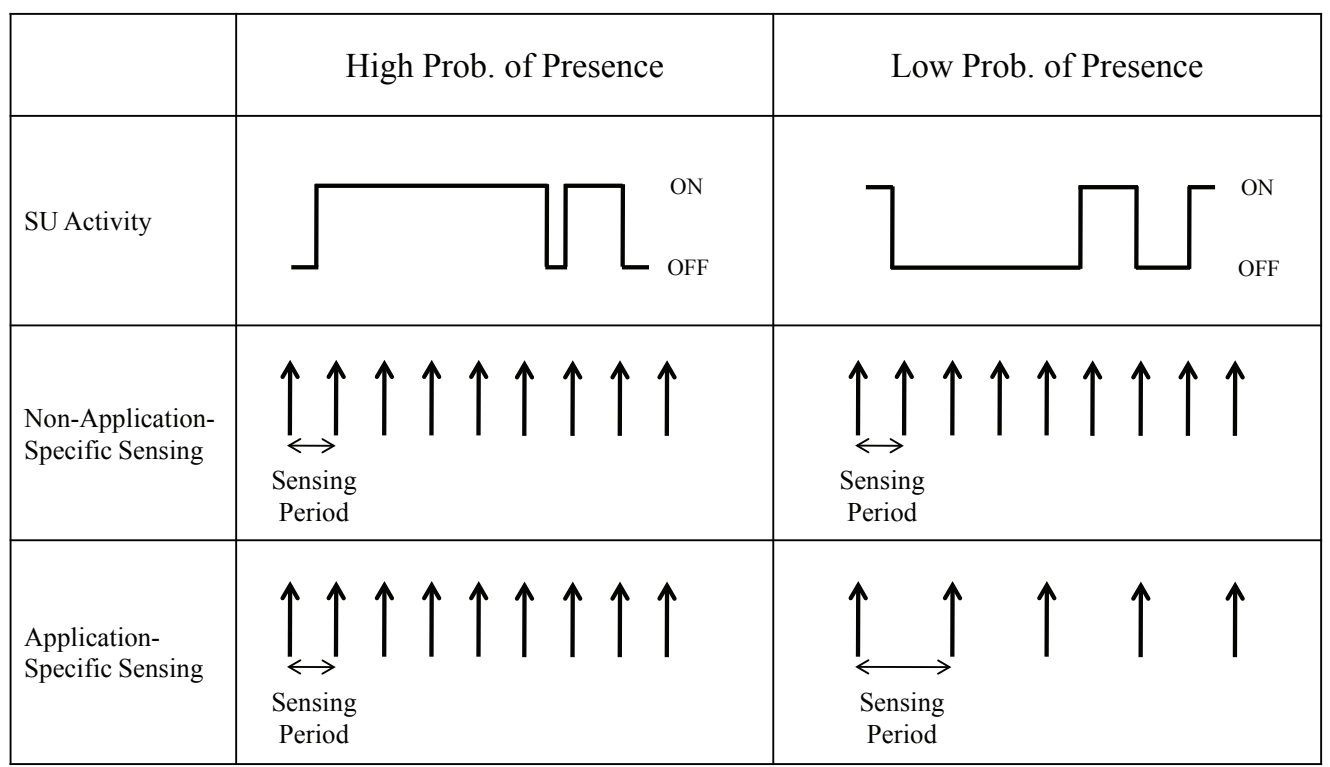

Figure 4.1: Non-application-specific spectrum sensing vs. application-specific spectrum sensing in CR. The PU's properties are assumed to be the same for the two types of applications.

simplicity of hardware implementation and low signal processing cost $[16,61-66]$. In this work, we choose energy detection method as our spectrum sensing method for a CSN. An energy detector measures the energy received on a primary channel and decides on the existence of the PU by comparing it with a system threshold. A basic hypothesis model for the model can be defined as follows [21]:

$$
r_{i}(n)= \begin{cases}n_{i}(n) & : H_{0} \\ k_{i}(n) s(n)+n_{i}(n) & : H_{1}\end{cases}
$$

where $r_{i}(n)$ is the discrete received signal at the $i^{\text {th }}$ secondary user during the $n^{\text {th }}$ sensing 
task, $s(n)$ is the transmitted signal by the PU, $n_{i}(n)$ is the additive white Gaussian noise at the $i^{\text {th }} \mathrm{SU}$, and $k_{i}(n)$ is the channel gain of the sensing channel between the $i^{\text {th }} \mathrm{SU}$ and the PU. Both $n_{i}(n)$ and $s(n)$ are assumed to be real-value and i.i.d. random processes with zero mean and variance $\sigma_{n}^{2}$ and $\sigma_{s}^{2}$ respectively. Moreover, the transmitted signal is assumed to be independent of the noise $n_{i}(n) . H_{0}$ is a null hypothesis, which states that the $\mathrm{PU}$ is absent in a certain spectrum band. On the other hand, $H_{1}$ is the alternative hypothesis, which indicates that there exists the licensed user's signal.

In this method, the received signal is first pre-filtered by an ideal bandpass filter, and the output of the filter is then squared and integrated over a time interval called observation time $T_{O}$ to produce the following test statistic:

$$
Y_{i}=\frac{1}{M} \sum_{n=1}^{M}\left|r_{i}(n)\right|^{2}
$$

where, $M$ is number of received samples within $T_{O}$. It can be shown that the test statistic $Y$ has a Chi-square distribution [67]. However, assuming the number of samples $(M)$ is large, the Chi-square distribution, based on the central limit theorem, can be closely approximated by a Gaussian distribution [68]. The test statistic is then as follows:

$$
Y \sim \begin{cases}\mathcal{N}\left(M \sigma_{n}^{2}, 2 M \sigma_{n}^{4}\right), & : H_{0} \\ \mathcal{N}\left(M\left(\sigma_{n}^{2}+\sigma_{s}^{2}\right), 2 M\left(\sigma_{n}^{2}+\sigma_{s}^{2}\right)^{2}\right) & : H_{1}\end{cases}
$$


where, $\mathcal{N}\left(\mu, \sigma^{2}\right)$ is the Gaussian distribution function with mean and variance $\mu$ and $\sigma^{2}$ respectively. In the above hypothesis testing, the probability of false alarm $P_{f}=\operatorname{Pr}[Y>$ $\left.\epsilon \mid H_{0}\right]$, and the probability of detection $P_{d}=\operatorname{Pr}\left[Y>\epsilon \mid H_{1}\right]$ can be expressed as follows:

$$
\begin{gathered}
P_{f}=Q\left(\frac{\epsilon-M \sigma_{n}^{2}}{\sigma_{n}^{2} \sqrt{2 M}}\right), \\
P_{d}=Q\left(\frac{\epsilon-M\left(\sigma_{s}^{2}+\sigma_{n}^{2}\right)}{\left(\sigma_{s}^{2}+\sigma_{n}^{2}\right) \sqrt{2 M}}\right)
\end{gathered}
$$

where $Q(x)=\frac{1}{\sqrt{2 \pi}} \int_{x}^{\infty} \exp \left(-\frac{u^{2}}{2}\right) d u$ is the Q-function, and $\epsilon$ is the decision threshold to decide on the presence or absence of the PU.

Note that in the energy detection method, the RF front-end cannot differentiate between the primary and secondary signals. In addition, it is assumed that SUs cannot perform the transmission and sensing tasks at the same time. Therefore, it is necessary to periodically perform the spectrum sensing task [12].

\subsection{Application-Specific Spectrum Sensing}

The proposed method is based on the continuous time Markov chain (CTMC) presented in Chapter 3. As shown in Fig. 4.2, the operation of both secondary and primary networks can be divided into three states. During the idle state, neither the secondary nor the primary network operates in the spectrum. The secondary network operates in 
the spectrum within the secondary network transmission state, and the PU operates in the spectrum within the primary user transmission state. We assume without loss of generality a homogenous CSN with $N$ secondary users, where each SU has the same arrival rates $\lambda_{1}=\lambda_{2}=\cdots \lambda_{N}=\lambda_{s}$ and also has the same departure rates $\mu_{1}=\mu_{2}=$ $\cdots \mu_{N}=\mu_{s}$. Hence, the probability of being at the idle state $\Pi_{0}$, secondary network transmission state $\Pi_{s}$, and the primary user transmission state $\Pi_{P}$ are given by (see Chapter 3):

$$
\begin{gathered}
\Pi_{0}=\frac{\mu_{P}}{\left(\mu_{P}+\lambda_{P}\right)\left(1+\frac{N \lambda_{s}}{\mu_{s}+\lambda_{P}}\right)}, \\
\Pi_{s}=\frac{\lambda_{s}}{\mu_{s}+\lambda_{P}} \Pi_{0}, \\
\Pi_{P}=\frac{\lambda_{P}}{\mu_{P}+\lambda_{P}} .
\end{gathered}
$$

During the secondary network transmission state, the secondary network must stop data transmission every $T_{T}$ seconds and perform the sensing task. If the result of the sensing task suggests that the PU is present, the secondary network must immediately vacate the spectrum. However, if the sensing task suggests that the PU is still absent, the secondary network will continue to operate in the spectrum for another $T_{T}$ seconds. The sensing period $\left(T_{P T}\right)$ in the secondary network transmission state is then obtained by

$$
T_{P T}=T_{O}+T_{T}
$$




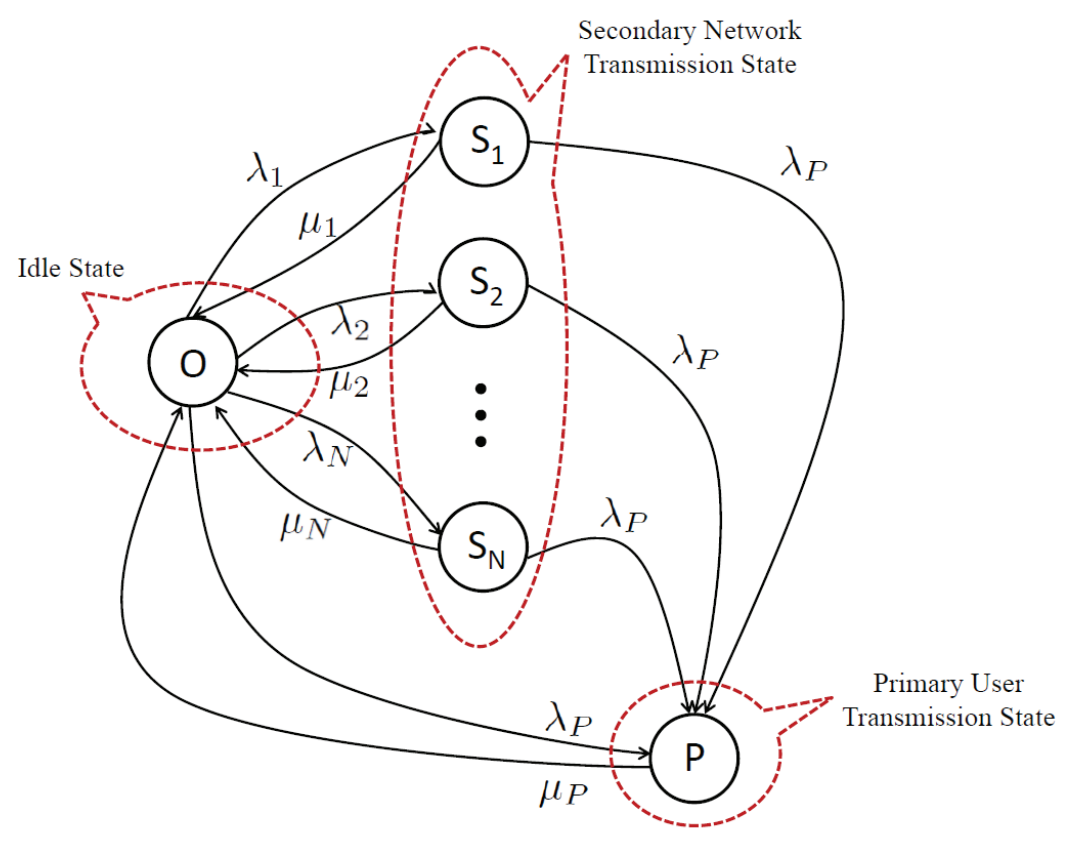

Figure 4.2: The operation of the CSN is divided into three states: idle, secondary network transmission, and primary user transmission.

where $T_{O}$ is the observation time.

If the CSN is at the idle state, the sensing task is performed every $T_{P I}$ seconds to detect the spectrum opportunities. Therefore, the $T_{P I}$ is given by

$$
T_{P I}=T_{O}+T_{I}
$$

\subsubsection{Problem Definitions}

- Definition 1: During the secondary network transmission state, the interference ratio $R_{I}$ is defined as the expected fraction of the primary user transmission state interrupted by the transmission of the secondary network, which will be derived 
in (4.19). The interference ratio threshold $R_{I}^{\text {th }}$ is a predefined system parameter which denotes the maximum acceptable $R_{I}$.

- Definition 2: Within the idle state, the undetected opportunity ratio $R_{U}$ is defined as the expected fraction of the spectrum opportunity undetected by the secondary network, which will be derived in (4.24). The undetected opportunity threshold $R_{U}^{t h}$ is a predefined system parameter that denotes the maximum acceptable $R_{U}$.

\subsubsection{Derivation of $R_{I}$ and $R_{U}$}

In this section, we derive the interference ratio and undetected opportunity ratio for the proposed method. Fig. 4.3 depicts a tree-diagram to fully illustrate the situations where a partial or complete interference, undetected opportunity, or secondary network transmission may occur ${ }^{1}$.

In Fig. 4.3, the probability that the PU operates in the spectrum is denoted by $\Pi_{P}$. Therefore, the probability that the PU does not operate in the spectrum is $\left(1-\Pi_{P}\right)$. In addition, the probability that the secondary network is at the transmission state is given by $\sum_{i=1}^{N} \Pi_{i}$. Thus, the probability that the secondary network does not operate in the spectrum is given by $\left(1-\sum_{i=1}^{N} \Pi_{i}\right)$. The hypothesis testing probabilities will be as follows:

\footnotetext{
${ }^{1}$ We assume that the secondary users share the licensed channel with a predefined medium access control protocol.
} 


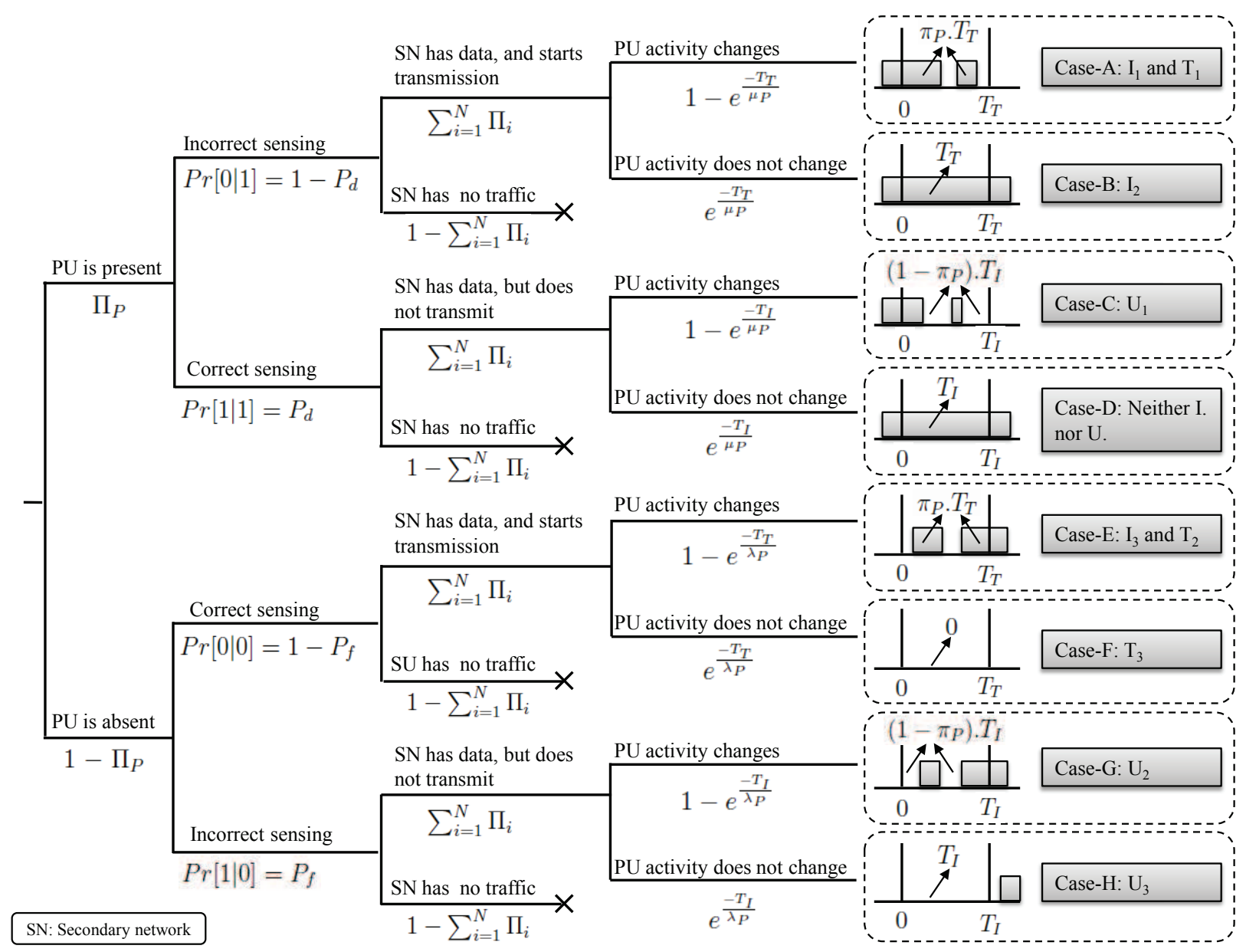

Figure 4.3: Illustration of cases of interference, undetected spectrum opportunity, and interference-free transmission time in the hybrid spectrum sensing. 
- $P_{d}$ is the probability that the sensing result suggests that the $\mathrm{PU}$ is present, given that the PU is present.

- $\operatorname{Pr}[0 \mid 1]=1-P_{d}$ is the probability that the sensing result suggests that the $\mathrm{PU}$ is absent, given that the PU is present.

- $P_{f}$ is the probability that the sensing result suggests that the PU is present, given that the PU is absent.

- $\operatorname{Pr}[0 \mid 0]=1-P_{f}$ is the probability that the sensing result suggests that the $\mathrm{PU}$ is absent, given that the PU is absent.

The probability that the PU's state varies within the transmission time $T_{T}$ is also derived. If the PU is present, the probability that it continues to operate in the spectrum for $T_{T}$ seconds is

$$
\begin{array}{r}
\operatorname{Pr}\left[t>T_{T} \mid P U: \text { present }\right]=1-\operatorname{Pr}\left[t \leq T_{T} \mid P U: \text { present }\right] \\
=1-\left(1-e^{-\frac{T_{T}}{\mu_{P}}}\right)=e^{-\frac{T_{T}}{\mu_{P}}} .
\end{array}
$$

If the PU is present, the probability of at least one transition of the PU's state within $T_{T}$ seconds is the complement event of (4.11). Therefore,

$$
\operatorname{Pr}\left[t<T_{T} \mid P U: \text { present }\right]=1-e^{-\frac{T_{T}}{\mu_{P}}} .
$$


Similar to the above calculation, the probability that the PU's state varies within the idle time $T_{I}$ can be derived by substituting $T_{I}$ for $T_{T}$ in (4.11) and (4.12).

If the $\mathrm{PU}$ is absent, the probability that the $\mathrm{PU}$ continues to be absent for $T_{T}$ seconds is

$$
\operatorname{Pr}\left[t>T_{T} \mid P U: \text { absent }\right]=e^{-\frac{T_{T}}{\lambda_{P}}} .
$$

Therefore,, if the PU is absent, the probability of at least one transition of the PU's activity within $T_{T}$ seconds is the complement event of (4.13), as follows:

$$
\operatorname{Pr}\left[t<T_{T} \mid P U: \text { absent }\right]=1-e^{-\frac{T_{T}}{\lambda_{P}}} .
$$

Equations (4.13) and (4.14) hold for cases where the CSN is at the idle state by substituting $T_{I}$ for $T_{T}$.

As shown in Fig. 4.3, the secondary network will interrupt the primary user's activity at Case-A, Case-B and Case-E, as follows: The PU is operating in the spectrum, but the secondary network's sensing result suggests that the PU is absent (incorrect sensing result). In addition, the secondary network has data to send and starts data transmission for $T_{T}$ seconds. This will cause interference with the primary network. Based on the PU's activity, two cases can occur as follows:

- Fig. 4.3 Case-A: If the PU's state changes at least once during the transmission 
time $\left(T_{T}\right)$, a partial interference happens $\left(I_{1}\right)$. Therefore, the expected interference in this scenario can be expressed as

$$
E\left[I_{1}\right]=\Pi_{P} \operatorname{Pr}[0 \mid 1]\left(\sum_{i=1}^{N} \Pi_{i}\right)\left(1-e^{-\frac{T_{T}}{\mu_{P}}}\right) \Pi_{P} T_{T}
$$

- Fig. 4.3 Case-B: If the PU's state does not change during the transmission time, a complete interference occurs $\left(I_{2}\right)$, and the secondary network will interfere with the PU during the whole transmission period ( $T_{T}$ seconds). Therefore, the expected interference in this case is given by

$$
E\left[I_{2}\right]=\Pi_{P} \operatorname{Pr}[0 \mid 1]\left(\sum_{i=1}^{N} \Pi_{i}\right)\left(e^{-\frac{T_{T}}{\mu_{P}}}\right) T_{T}
$$

A partial interference also happens under the following scenario: The PU is not operating in the spectrum, and the secondary network correctly detects the absence of the PU. In addition, the secondary network has data to send; therefore it starts data transmission for $T_{T}$ seconds. However, the PU's state varies from the OFF state to the ON state during the transmission time, and causes a partial interference. Hence, the expected interference when the PU is initially absent during the transmission time $T_{T}$, 
can be expressed as

$$
E\left[I_{3}\right]=\left(1-\Pi_{P}\right) \operatorname{Pr}[0 \mid 0]\left(\sum_{i=1}^{N} \Pi_{i}\right)\left(1-e^{-\frac{T_{T}}{\lambda_{P}}}\right) \Pi_{P} T_{T}
$$

Finally, we obtain the interference ratio $R_{I}$ as follows:

$$
R_{I}=\frac{E\left[I_{1}\right]+E\left[I_{2}\right]+E\left[I_{3}\right]}{T_{T} \Pi_{P}}
$$

where $E\left[I_{1}\right], E\left[I_{2}\right]$, and $E\left[I_{3}\right]$ are derived in (4.15), (4.16), and (4.17) respectively. After some algebraic manipulation, $R_{I}$ is obtained by

$$
R_{I}=\left(\sum_{i=1}^{N} \Pi_{i}\right)\left\{\left(1-P_{d}\right)\left(e^{-\frac{T_{T}}{\mu_{P}}}\left(1-\Pi_{P}\right)+\Pi_{P}\right)+\left(1-\Pi_{P}\right)\left(1-P_{f}\right)\left(1-e^{-\frac{T_{T}}{\lambda_{P}}}\right)\right\}
$$

In addition, as depicted in Fig. 4.3, the spectrum opportunities will be undetected by the secondary network at the following circumstances:

- Fig. 4.3 Case-C: The PU is operating in the spectrum, and the secondary network correctly detects the presence of the PU. The secondary network has data to send, but it cannot start data transmission due to the result of the spectrum sensing. Therefore, the secondary network goes to the idle state for $T_{I}$ seconds. However, the PU's state changes from the ON state to the OFF state within this period, so 
the new opportunity will be undetected $\left(U_{1}\right)$. The expected undetected spectrum opportunity in this case can be expressed as follows:

$$
E\left[U_{1}\right]=\Pi_{P} \operatorname{Pr}[1 \mid 1]\left(\sum_{i=1}^{N} \Pi_{i}\right)\left(1-e^{-\frac{T_{i}}{\mu_{P}}}\right)\left(1-\Pi_{P}\right) T_{I} .
$$

There are also undetected spectrum opportunities at the following scenarios: The PU is not operating in the spectrum, but the secondary network suggests that the $\mathrm{PU}$ is present (incorrect sensing result). In addition, the secondary network has data to send, but it cannot perform data transmission due to the spectrum sensing result. Therefore, the secondary network goes to the idle state for $T_{I}$ seconds. Based on the PU's activity, there will be two cases:

- Fig. 4.3 Case-G, PU's state changes during the idle time $\left(T_{I}\right)$. Hence, a partial spectrum opportunity will occur $\left(U_{2}\right)$. The expected undetected spectrum opportunity in this case can be expressed as follows:

$$
E\left[U_{2}\right]=\left(1-\Pi_{P}\right) \operatorname{Pr}[1 \mid 0]\left(\sum_{i=1}^{N} \Pi_{i}\right)\left(e^{-\frac{T_{i}}{\lambda_{P}}}\right) T_{I} .
$$

- Fig. 4.3 Case-H: The PU's state does not change within the idle time $\left(T_{I}\right)$, and the entire spectrum opportunity will be undetected $\left(U_{3}\right)$. The expected undetected 
spectrum opportunity in this case is given by

$$
E\left[U_{3}\right]=\left(1-\Pi_{P}\right) \operatorname{Pr}[0 \mid 0]\left(\sum_{i=1}^{N} \Pi_{i}\right)\left(1-e^{-\frac{T_{T}}{\lambda_{P}}}\right) \Pi_{P} T_{T}
$$

We obtain the undetected opportunity ratio $R_{U}$ as follows:

$$
R_{U}=\frac{E\left[U_{1}\right]+E\left[U_{2}\right]+E\left[U_{3}\right]}{\left(1-\Pi_{P}\right) T_{I}}
$$

where $E\left[U_{1}\right], E\left[U_{2}\right]$, and $E\left[U_{3}\right]$ are derived in (4.20), (4.21), and (4.22) respectively. After some algebraic manipulation, $R_{U}$ is derived by

$$
R_{U}=\left(\sum_{i=1}^{N} \Pi_{i}\right)\left\{P_{f}\left(\mu_{P} e^{-\frac{T_{I}}{\lambda_{P}}}+1-\Pi_{P}\right)+\Pi_{P} P_{d}\left(1-e^{-\frac{T_{I}}{\mu_{P}}}\right)\right\}
$$

\subsubsection{Throughput Analysis}

An important objective of dynamic spectrum allocation is to obtain a high throughput for the secondary network in a statistical sense. The more frequently the available spectrum is used by the secondary network, the more efficiently the CSN will perform. The maximal data rate of the $i^{\text {th }}$ secondary user is given by [69]

$$
r_{i}=W \log _{2}\left(1+\frac{P_{i} G_{i}}{n_{0}}\right)
$$


where $W$ is the communication bandwidth, $P_{i}$ is the transmission power of user $i, n_{0}$ is the power of the additive white Gaussian noise (AWGN), and $G_{i}$ is the channel gain for user $i$. Without loss of generality, we assume a homogenous CSN where all SUs achieve $r_{s}$, the maximal data rate of each $\mathrm{SU}$.

We consider the interference-free secondary network's transmission for accurate throughput analysis. As shown in Fig. 4.3, the secondary network transmission can occur in one of the following circumstances:

- Fig. 4.3 Case-A: The PU is operating in the spectrum, but the secondary network's sensing result suggests that the PU is absent (incorrect sensing result). Also, the secondary network has data to send. Based on the sensing result, therefore, the secondary network starts data transmission for $T_{T}$ seconds. This will cause interference with the PU's data transmission. However, if the PU's state changes during the transmission time, a partial interference-free secondary network's transmission happens $\left(T_{1}\right)$. Therefore, the expected interference-free transmission in this case can be expressed as follows:

$$
E\left[T_{1}\right]=\Pi_{P} \operatorname{Pr}[0 \mid 1]\left(\sum_{i=1}^{N} \Pi_{i}\right)\left(1-e^{-\frac{T_{T}}{\mu_{P}}}\right)\left(1-\Pi_{P} T_{T}\right) .
$$

In addition, there are interference-free transmission cases as follows: the PU is not 
operating in the spectrum, and the secondary network correctly detects the absence of the PU. In addition, the secondary network has data to send; therefore, it starts data transmission for $T_{T}$ seconds. Based on the PU's activity, two cases can occur:

- Fig. 4.3 Case-E: if the PU's state varies from the OFF to the ON state during the transmission time, a partial interference will happen. Hence, the expected interference-free transmission in this case is given by

$$
E\left[T_{2}\right]=\left(1-\Pi_{P}\right) \operatorname{Pr}[0 \mid 0]\left(\sum_{i=1}^{N} \Pi_{i}\right)\left(1-e^{-\frac{T_{T}}{\lambda_{P}}}\right)\left(1-\Pi_{P} T_{T}\right) .
$$

- Fig. 4.3 Case-F: a complete interference-free transmission can happen if the PU's state does not change during the transmission time $T_{T}$. The expected transmission in this case is obtained by

$$
E\left[T_{3}\right]=\left(1-\Pi_{P}\right) \operatorname{Pr}[0 \mid 0]\left(\sum_{i=1}^{N} \Pi_{i}\right)\left(e^{-\frac{T_{T}}{\lambda_{P}}}\right) T_{T} .
$$

After some algebraic manipulation, the average interference-free transmission time $E\left[T_{N I}\right]=E\left[T_{1}\right]+E\left[T_{2}\right]+E\left[T_{3}\right]$ is derived as,

$$
\begin{array}{r}
E\left[T_{N I}\right]=\left(\sum_{i=1}^{N} \Pi_{i}\right)\left\{\Pi_{P}\left(1-P_{d}\right)\left(1-e^{-\frac{T_{T}}{\mu_{P}}}\right)\left(1-\Pi_{P} T_{T}\right)+\left(1-\Pi_{p}\right)\left(1-P_{f}\right)\right. \\
\left.\times\left(\left(1-e^{-\frac{T_{T}}{\lambda_{P}}}\right)\left(1-\Pi_{P} T_{T}\right)+\left(e^{-\frac{T_{T}}{\lambda_{P}}}\right) \cdot T_{T}\right)\right\} .
\end{array}
$$


We define the average throughput of the secondary network as

$$
\Gamma_{s}=r_{s} E\left[T_{N I}\right]
$$

where $r_{s}$ is given in (4.25).

\subsubsection{Energy Consumption Analysis}

The secondary network's energy consumption consists of three elements: The energy consumption to perform the sensing task $E_{s}$, the energy consumption for the data transmission $E_{t}$, and the energy consumption during the idle state $E_{i d}$. In this work, we do not consider $E_{i d}$ as it is relatively small. Moreover, in contrast to the throughput analysis, where only the interference-free secondary network's transmission is considered, both interfered and non-interfered data transmission must be considered for the energy consumption calculation. In other words, both interfered and non-interfered data transmission will drain the secondary network's energy sources. As shown in Fig. 4.3, the probability that the secondary network's transmission state (both interfered and non- 
interfered) $\operatorname{Pr}[T]$ can be derived as

$$
\begin{array}{r}
\operatorname{Pr}[T]=\Pi_{P}\left(1-P_{d}\right)\left(\sum_{i=1}^{N} \Pi_{i}\right)+\left(1-\Pi_{P}\right)\left(1-P_{f}\right)\left(\sum_{i=1}^{N} \Pi_{i}\right) \\
=\left(\sum_{i=1}^{N} \Pi_{i}\right)\left(\Pi_{P}\left(1-P_{d}\right)+\left(1-\Pi_{P}\right)\left(1-P_{f}\right)\right) .
\end{array}
$$

Therefore, the total energy consumption of the secondary network $E=E_{s}+E_{t}$ is given by,

$$
E=\left(\frac{T_{\text {sim }}}{E\left[T_{P}\right]}\right) E_{s 0}+\left(T_{s i m} \cdot \operatorname{Pr}[T]\right) P_{t 0}
$$

where $E_{s 0}$ is the unit energy consumption for each sensing task $(J), P_{t 0}$ is the unit transmission power $(J / S)$, and $T_{\text {sim }}$ is the simulation time. In addition, the average of the sensing period $E\left[T_{P}\right]$ will be derived in (4.35). The total power consumption of the secondary network is obtained by $P=\frac{E}{t_{s i m}}$.

\subsubsection{Problem Formulation}

According to the CTMC model shown in Fig. 4.2, the secondary network will be either at the idle state or at the transmission state. Hence, the CSN's objectives will be set for each of these states as follows: 


\subsubsection{Secondary Network Transmission State}

If the secondary network is at the transmission state, the objective will be to maximize the duration of data transmission $T_{T}$. However, it is more likely to interfere with the PU when the transmission time increases. Therefore, $T_{T}$ is allowed to increase only until the interference ratio reaches a predefined threshold value $\left(R_{I}^{t h}\right)$. Hence, in this state, the spectrum sensing problem is defined as follows:

\section{Maximize: $T_{T}^{*}$}

Subject to: $R_{I} \leq R_{I}^{\text {th }}$

where $T_{T}^{*}$ is the optimal transmission time. Moreover, the optimum sensing period within the secondary network transmission state is then $T_{P T}^{*}=T_{O}+T_{T}^{*}$.

Note that $R_{I}$ is an increasing function with respect to $T_{T}$; i.e., $\frac{d R_{I}}{d T_{T}}>0$. Hence, at the maximum $T_{T}$, the interference ratio will be equal to the largest possible amount $\left(R_{I}^{t h}\right)$. Therefore, an exhaustive search over $T_{T}$ from $T_{O}$ to the first value that satisfies $R_{I} \leq R_{I}^{t h}$ is employed to find $T_{T}^{*}$.

Proposition 1: $R_{I}$ is a monotonically increasing function of $T_{T}$.

Proof: Differentiate $R_{I}$ w.r.t. $T_{T}$; after some algebraic manipulation we have,

$$
\left(\frac{1-P_{f}}{1-P_{d}}\right)\left(\frac{\mu_{P}}{\lambda_{P}}\right) e^{\left(\frac{1}{\mu_{P}}-\frac{1}{\lambda_{P}}\right) T_{T}}>0
$$


Therefore, $R_{I}$ is a monotonically increasing function of $T_{T}$.

\subsubsection{Secondary Network Idle State}

If the CSN is at the idle state, the objective is to maximize the duration of the idle time $T_{I}$. It is expected that the energy consumption associated with the sensing task will be reduced by decreasing the sensing frequency. However, a large idle time increases the chance of missing spectrum opportunities. Therefore, $T_{I}$ can only increase when the undetected opportunity ratio is below the predefined threshold $\left(R_{U}^{\text {th }}\right)$. The spectrum sensing problem in this case is then defined as

Maximize: $T_{I}^{*}$

Subject to: $R_{U} \leq R_{U}^{t h}$

where, $T_{I}^{*}$ is the optimal idle time. In addition, the optimum sensing period when the secondary network is at the idle state will be $T_{P I}^{*}=T_{O}+T_{I}^{*}$.

Similar to the previous case, $R_{U}$ is an increasing function with respect to $T_{I}$; i.e., $\frac{d R_{U}}{d T_{I}}>0$. Hence, at the maximum $T_{I}$, the undetected opportunity ratio will have the largest possible value $\left(R_{U}^{t h}\right)$. Therefore, an exhaustive search over $T_{I}$ from $T_{O}$ to the first value that satisfies $R_{U} \leq R_{U}^{\text {th }}$ can be employed to find $T_{I}^{*}$.

Proposition 2: $R_{U}$ is a monotonically increasing function of $T_{I}$. 
Proof: Differentiate $R_{U}$ w.r.t. $T_{I}$; after some algebraic manipulation we have

$$
\left(\frac{P_{d}}{P_{f}}\right)\left(\frac{\lambda_{P}}{\mu_{P}}\right) e^{\left(\frac{1}{\lambda_{P}}-\frac{1}{\mu_{P}}\right) T_{I}}>0 .
$$

Therefore, $R_{U}$ is a monotonically increasing function of $T_{I}$.

\subsubsection{Optimum Sensing Period}

As noted before, the secondary network is at the idle state with the probability of $\Pi_{0}$, and at the transmission state with the probability of $\sum_{i=1}^{N} \Pi_{i}$. Therefore, the average of the optimum sensing period is given by,

$$
E\left[T_{P}^{*}\right]=\Pi_{0} T_{P I}^{*}+\left(\sum_{i=1}^{N} \Pi_{i}\right) T_{P T}^{*} .
$$

\subsection{Non-Application-Specific Spectrum Sensing}

In this section, a non-application-specific approach for spectrum sensing is presented for comparison purpose. In contrast with the hybrid method, where the properties of both PU and SUs are considered, a non-hybrid method solely considers the properties of the PU to find the optimal sensing period. Since the properties of SUs are unknown in this method, it is assumed that in an event of interference, SUs interfere with the PU over 
the entire transmission time. Moreover, SUs miss the entire opportunity in an event of undetected spectrum opportunity. Fig. 4.4 depicts a tree-diagram to illustrate when interference and undetected spectrum opportunities happen in the non-hybrid scheme.

Similar to the hybrid method, the interference ratio $R_{I}^{N H}$ and the undetected opportunity ratio $R_{U}^{N H}$ in the non-hybrid method can be derived as follows:

$$
\begin{gathered}
R_{I}^{N H}=\left(1-P_{d}\right)\left(e^{-\frac{T_{T}}{\mu_{P}}}\left(1-\Pi_{P}\right)+\Pi_{P}\right)+\left(1-\Pi_{P}\right)\left(1-P_{f}\right)\left(1-e^{-\frac{T_{T}}{\lambda_{P}}}\right), \\
R_{U}^{N H}=P_{f}\left(\mu_{P} e^{-\frac{T_{I}}{\lambda_{P}}}+1-\Pi_{P}\right)+\Pi_{P} P_{d}\left(1-e^{-\frac{T_{I}}{\mu_{P}}}\right) .
\end{gathered}
$$

In addition, similar to the hybrid method, the average throughput of the secondary network can be written as

$$
\Gamma_{s}^{N H}=r_{s} E\left[T_{N I}^{N H}\right]
$$

where $r_{s}$ is given in (4.25), and $E\left[T_{N I}^{N H}\right]$ is derived by

$$
\begin{array}{r}
E\left[T_{N I}^{N H}\right]=\Pi_{P}\left(1-P_{d}\right)\left(1-e^{-\frac{T_{T}}{\mu_{P}}}\right)\left(1-\Pi_{P} T_{T}\right) \\
+\left(1-\Pi_{p}\right)\left(1-P_{f}\right)\left(\left(1-e^{-\frac{T_{T}}{\lambda_{P}}}\right)\left(1-\Pi_{P} T_{T}\right)+\left(e^{-\frac{T_{T}}{\lambda_{P}}}\right) \cdot T_{T}\right) .
\end{array}
$$

Moreover, similar to the hybrid method, the total energy consumption of the sec- 


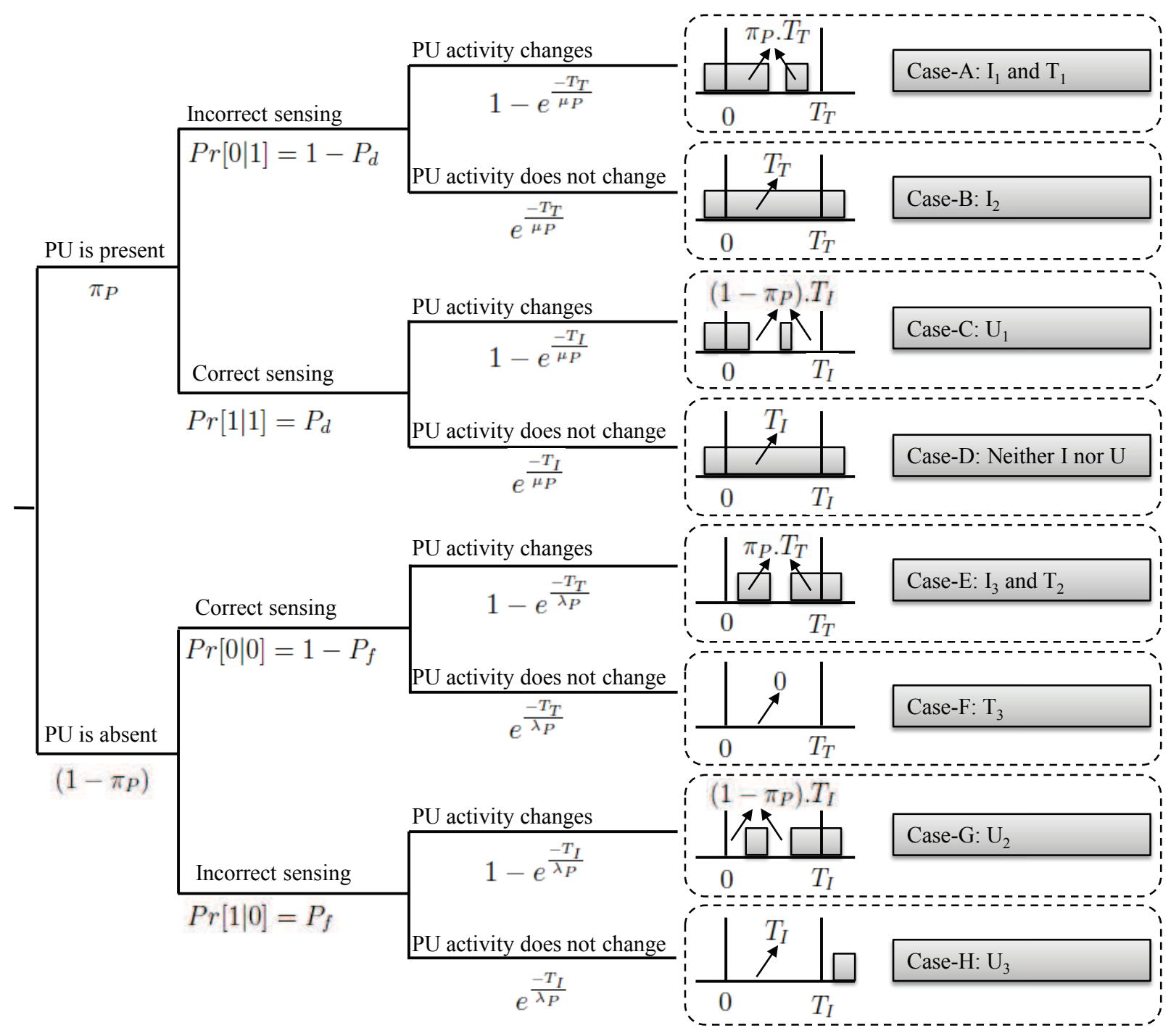

Figure 4.4: Illustration of the events of interference, undetected spectrum opportunity, and interference-free transmission time in the non-hybrid spectrum sensing. 
ondary network in the non-hybrid method $E^{N H}=E_{s}^{N H}+E_{t}^{N H}$ can be obtained by

$$
E^{N H}=\left(\frac{T_{s i m}}{E\left[T_{P}^{N H}\right]}\right) E_{s 0}+\left(T_{s i m} \cdot \operatorname{Pr}\left[T^{N H}\right]\right) P_{t 0}
$$

where $E\left[T_{P}^{N H}\right]=\left(1-\Pi_{P}\right) T_{P I}+\Pi_{P} T_{P T}$, and $\operatorname{Pr}\left[T^{N H}\right]=\Pi_{P}\left(1-P_{d}\right)+\left(1-\Pi_{P}\right)\left(1-P_{f}\right)$.

The total power consumption of the secondary network in this case is obtained by $P^{N H}=$ $\frac{E^{N H}}{T_{s i m}}$

\subsection{Numerical and Simulation Results}

We consider a homogenous CSN with two SUs $(N=2)$, and compare three CR networks with different probabilities of presence $\left(\Pi_{s 1}<\Pi_{s 2}<\Pi_{s 3}\right)$. The arbitrary probabilities of presence are selected as follows: $\Pi_{s 1}=0.24, \Pi_{s 2}=0.28$, and $\Pi_{s 3}=0.31$.

\subsubsection{Interference Ratio Analysis}

Fig. 4.5 depicts the interference ratio curves for the secondary network 1, secondary network 2 , and secondary network 3 along with the optimum transmission time $T_{T}^{*}$ for each network. As shown, $T_{T}^{*}$ is decreased when the secondary network's probability of presence is increased. This is due to fact that the interference ratio will sooner exceed the $R_{I}^{\text {th }}$ for a secondary network with a greater $\Pi_{s}$. In addition, the proposed method's 
performance is compared with the non-hybrid method. As depicted, the interference ratio in the non-hybrid method $R_{I}^{N H}$ is identical for all the secondary networks as the SU's properties are not considered in the non-hybrid method. Moreover, the proposed method manages to increase the SU's optimum transmission time $T_{T}^{*}$, as compared with the non-hybrid method to improve the secondary network's spectrum usage.

\subsubsection{Undetected Opportunity Ratio Analysis}

Similar to the previous simulation, three secondary networks are considered that have different probabilities of presence $\Pi_{s 1}<\Pi_{s 2}<\Pi_{s 3}$. Fig. 4.6 depicts the undetected opportunity ratio curves for these networks along with the optimum idle time $T_{I}^{*}$ for each network. As expected, the optimum idle time is decreased in a secondary network that has a greater $\Pi_{s}$ to response to the demand for more spectrum usage in such a network. In addition, the proposed method's performance is compared with the nonhybrid approach. In the first place, the undetected opportunity ratio in the non-hybrid method $R_{U}^{N H}$ is identical for all the above networks, as $R_{U}^{N H}$ is not a function of the secondary network's parameters. In the second place, the proposed method manages to increase $T_{I}^{*}$, as compared with the non-hybrid method to reduce the frequency of the spectrum sensing, and thus to reduce the energy consumption associated with the sensing task. 


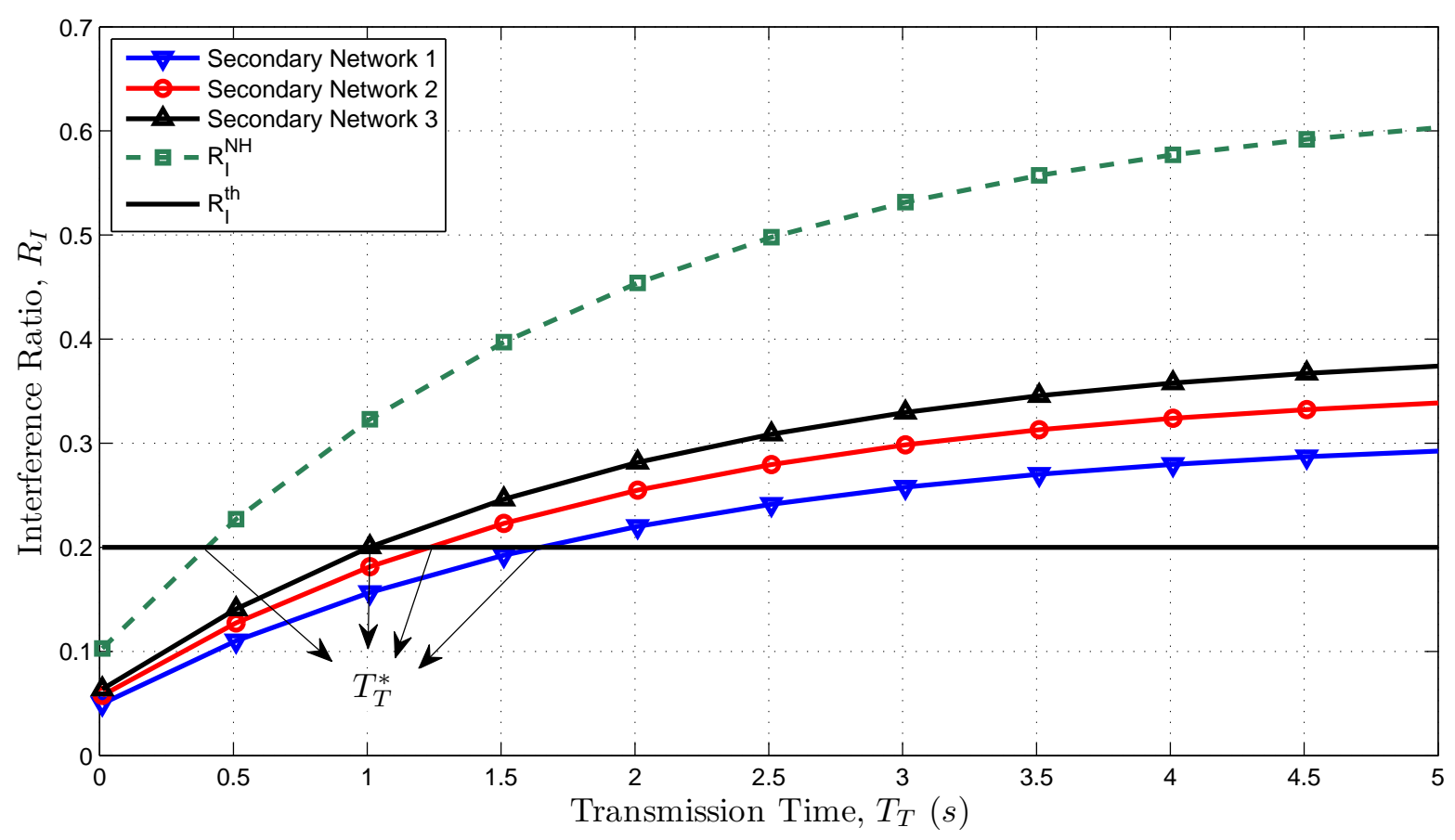

Figure 4.5: Interference ratio for the secondary network 1, 2, and $3\left(\Pi_{s 1}<\Pi_{s 2}<\Pi_{s 3}\right.$ and $R_{I}^{\text {th }}=0.2$ ).

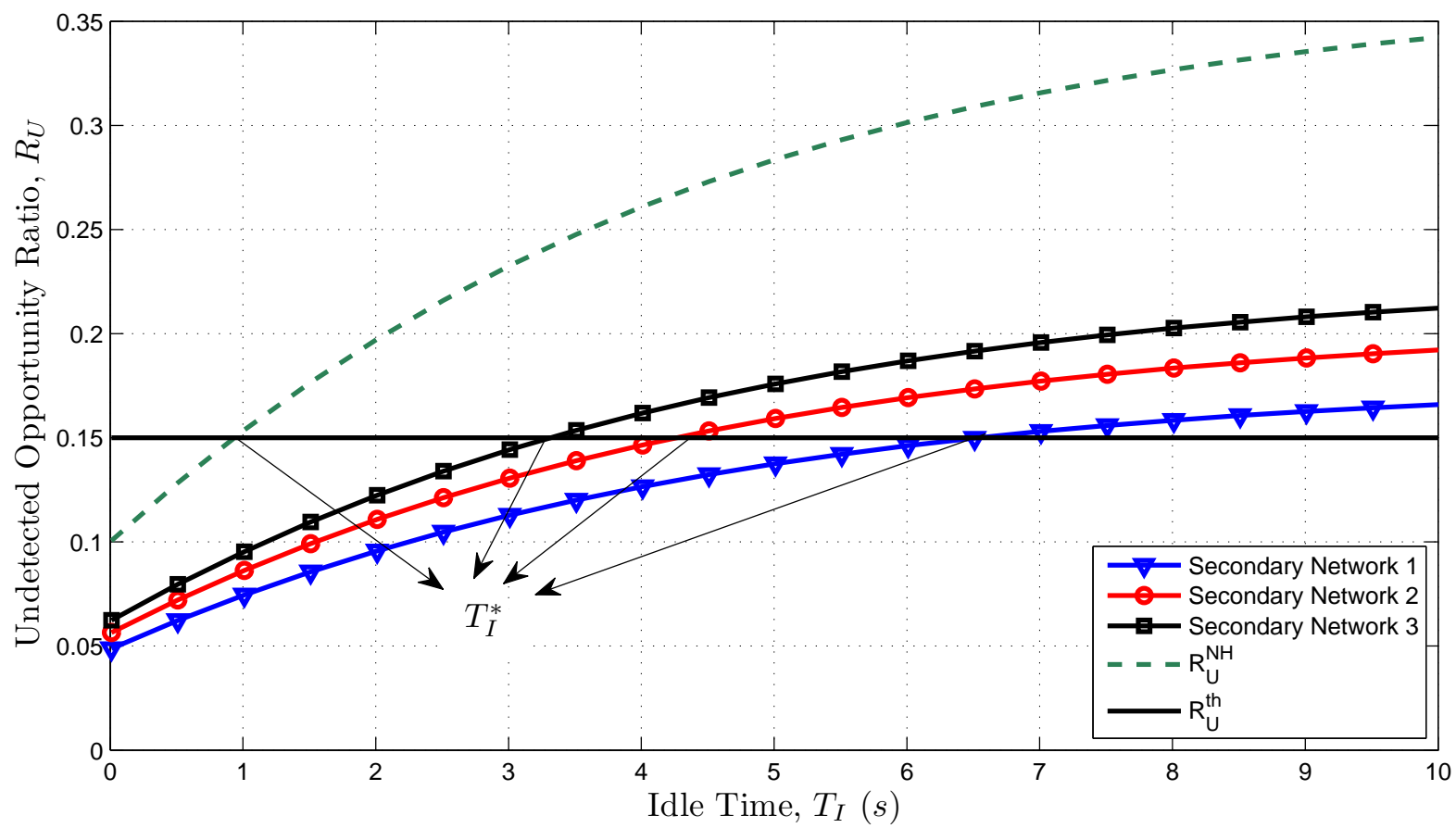

Figure 4.6: Undetected opportunity ratio for the secondary network 1, 2, and $3\left(\Pi_{s 1}<\right.$ $\Pi_{s 2}<\Pi_{s 3}$ and $\left.R_{U}^{t h}=0.15\right)$. 


\subsubsection{Optimum Sensing Period Analysis}

In this section, we study the optimum sensing period for two cases. First, the secondary network's departure rate $\mu_{s}$ is assumed to be fixed, and the secondary network's arrival rate $\lambda_{s}$ varies (Case-I). Second, the secondary network's arrival rate $\lambda_{s}$ is fixed, and the secondary network's departure rate $\mu_{s}$ varies (Case-II).

\section{Case-I: Fixed $\mu_{s}$, Variable $\lambda_{s}$}

Fig. 4.7 depicts the average of the optimum sensing period $E\left[T_{P}^{*}\right]$ in Case-I. As shown, $E\left[T_{P}^{*}\right]$ is decreased when $\lambda_{s}$ is increased. This is due to the fact that the secondary network's probability of presence is also increased when $\lambda_{s}$ is increased. Therefore, in the proposed method, the optimum sensing period is decreased to provide greater sensing frequency (smaller $E\left[T_{P}^{*}\right]$ ) in response to the higher demand for spectrum usage in a secondary network with greater $\lambda_{s}$.

In addition, $E\left[T_{P}^{*}\right]$ is compared for different values of $\mu_{s}$. As depicted in Fig. 4.7, $E\left[T_{P}^{*}\right]$ is increased when $\mu_{s}$ is increased. This is due to the fact that for a fixed value of $\lambda_{s}$, the secondary network's probability of presence $\Pi_{s}$ is decreased when $\mu_{s}$ is increased. Therefore, $E\left[T_{P}^{*}\right]$ is increased to reduce the sensing frequency (larger $E\left[T_{P}^{*}\right]$ ) and thus to reduce the energy consumption associated with the sensing task. 


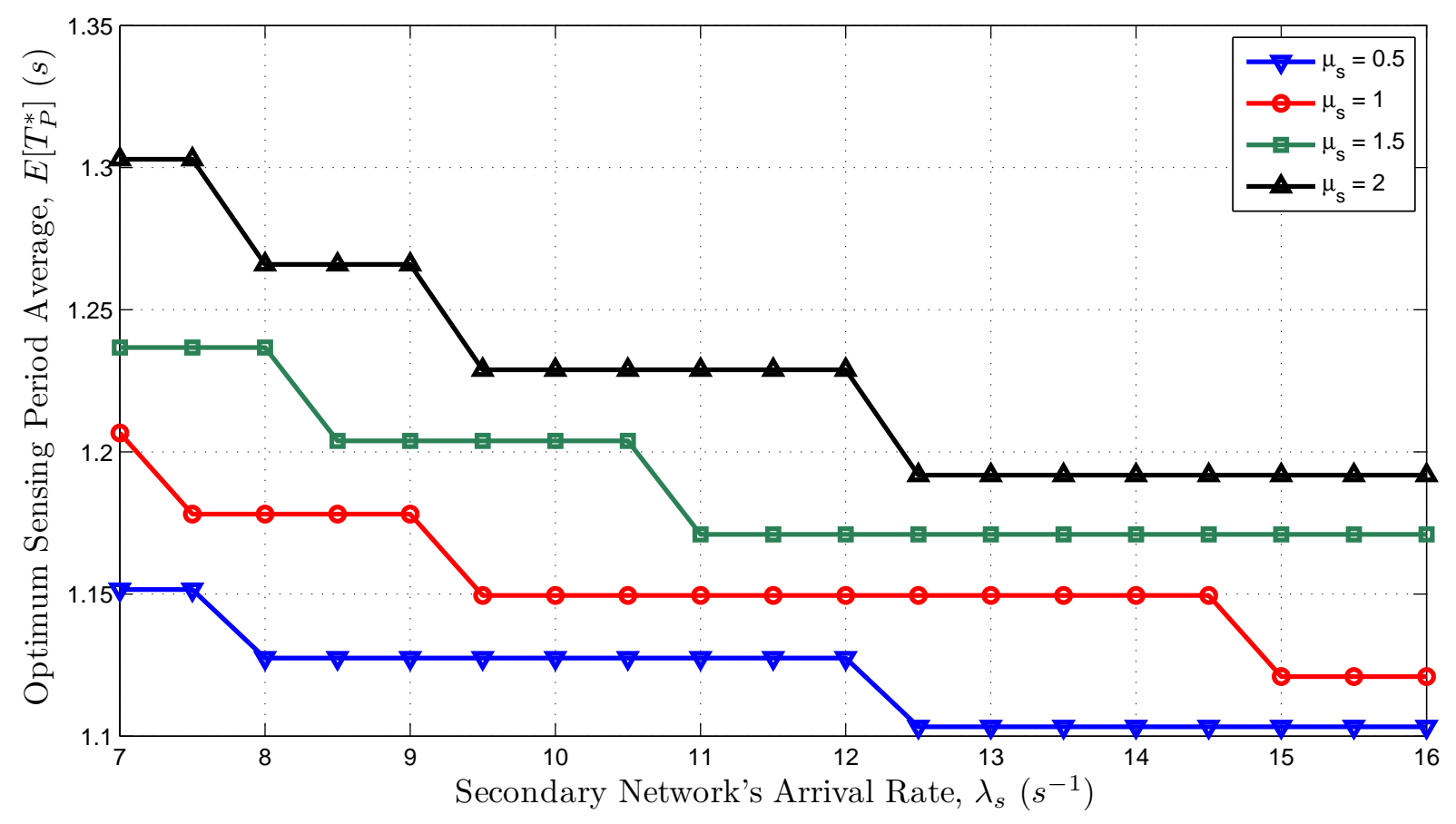

Figure 4.7: The average of the optimum sensing period in Case-I.

\section{Case-II: Fixed $\lambda_{s}$, Variable $\mu_{s}$}

Fig. 4.8 depicts the average of the optimum sensing period $E\left[T_{P}^{*}\right]$ in Case-II. As shown, $E\left[T_{P}^{*}\right]$ is increased when $\mu_{s}$ is increased. This is due to the fact that $\Pi_{s}$ is decreased when $\mu_{s}$ is increased (see (4.7) and (4.6)). Therefore, in the proposed method, the optimum sensing period is increased to provide smaller sensing frequency (larger $E\left[T_{P}^{*}\right]$ ). Hence, the energy consumption associated with the sensing task will be reduced in a secondary network with an smaller $\Pi_{s}$.

Furthermore, $E\left[T_{P}^{*}\right]$ is compared for different values of $\lambda_{s}$. As depicted in Fig. 4.8, $E\left[T_{P}^{*}\right]$ is increased when $\lambda_{s}$ is decreased. This is due to the fact that for a fixed value of 
$\mu_{s}$, the secondary network's probability of presence $\Pi_{s}$ is decreased when $\lambda_{s}$ is decreased. Therefore, $E\left[T_{P}^{*}\right]$ is increased to reduce the sensing frequency (larger $E\left[T_{P}^{*}\right]$ ) and thus to reduce the energy consumption associated with the sensing task.

\subsubsection{Throughput and Power Consumption Analysis}

Fig. 4.9 depicts the average throughput $\Gamma_{s}$ for two cognitive networks. As shown, $\Gamma_{s}$ is increased when $T_{T}$ is increased. Therefore, the optimum $T_{T}^{*}$ yields to the maximum $\Gamma_{s}$ for each secondary network. Each curve, therefore, is stopped at its corresponding $T_{T}^{*}$ to avoid exceeding the $R_{I}$ threshold. In addition, for a fixed value of $T_{T}, \Gamma_{s}$ is greater for a secondary network that has a greater probability of presence $\Pi_{s}$, i.e, $\Gamma_{2}>\Gamma_{1}$ when $\Pi_{2}>\Pi_{1}$. The proposed method is also compared with the non-hybrid approach. As shown, the proposed method achieves up to a $11 \%$ greater throughput for the secondary network 2, as compared with the non-hybrid scheme.

Fig. 4.10 depicts the total power consumption $(P)$ of the secondary networks 1 and 2 . In the first place, $P$ is decreased when $T_{I}$ is increased, as expected. Hence, the optimum $T_{I}^{*}$ yields to the minimum power consumption for each secondary network. Each curve, therefore, is stopped at its corresponding $T_{I}^{*}$ to avoid exceeding the $R_{U}$ threshold. In the second place, $P$ is increased when the secondary network's probability of presence $\Pi_{s}$ is increased, i.e, $P_{2}>P_{1}$ when $\Pi_{2}>\Pi_{1}$. Finally, the proposed method is compared with 


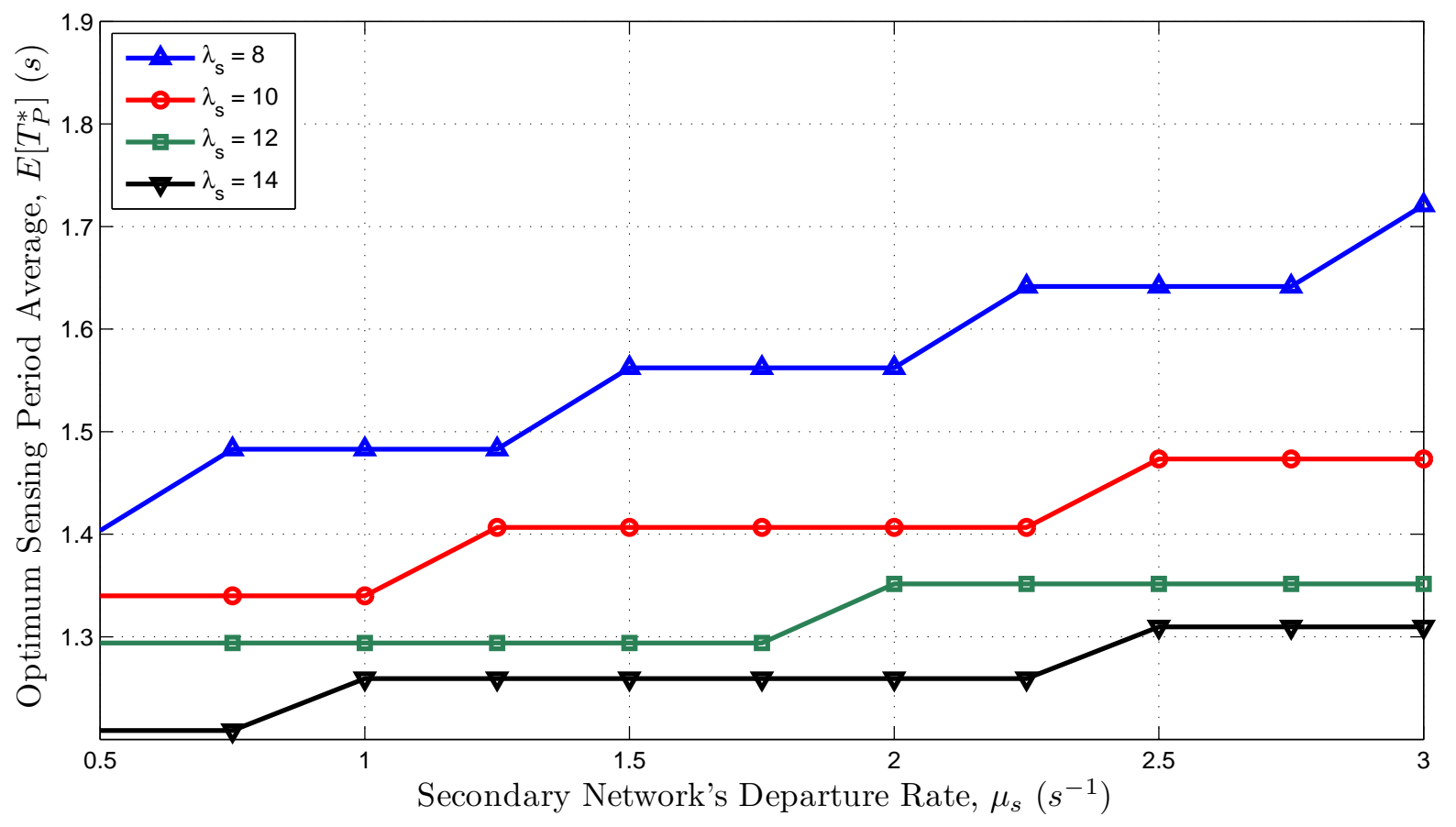

Figure 4.8: The average of the optimum sensing period in Case-II.

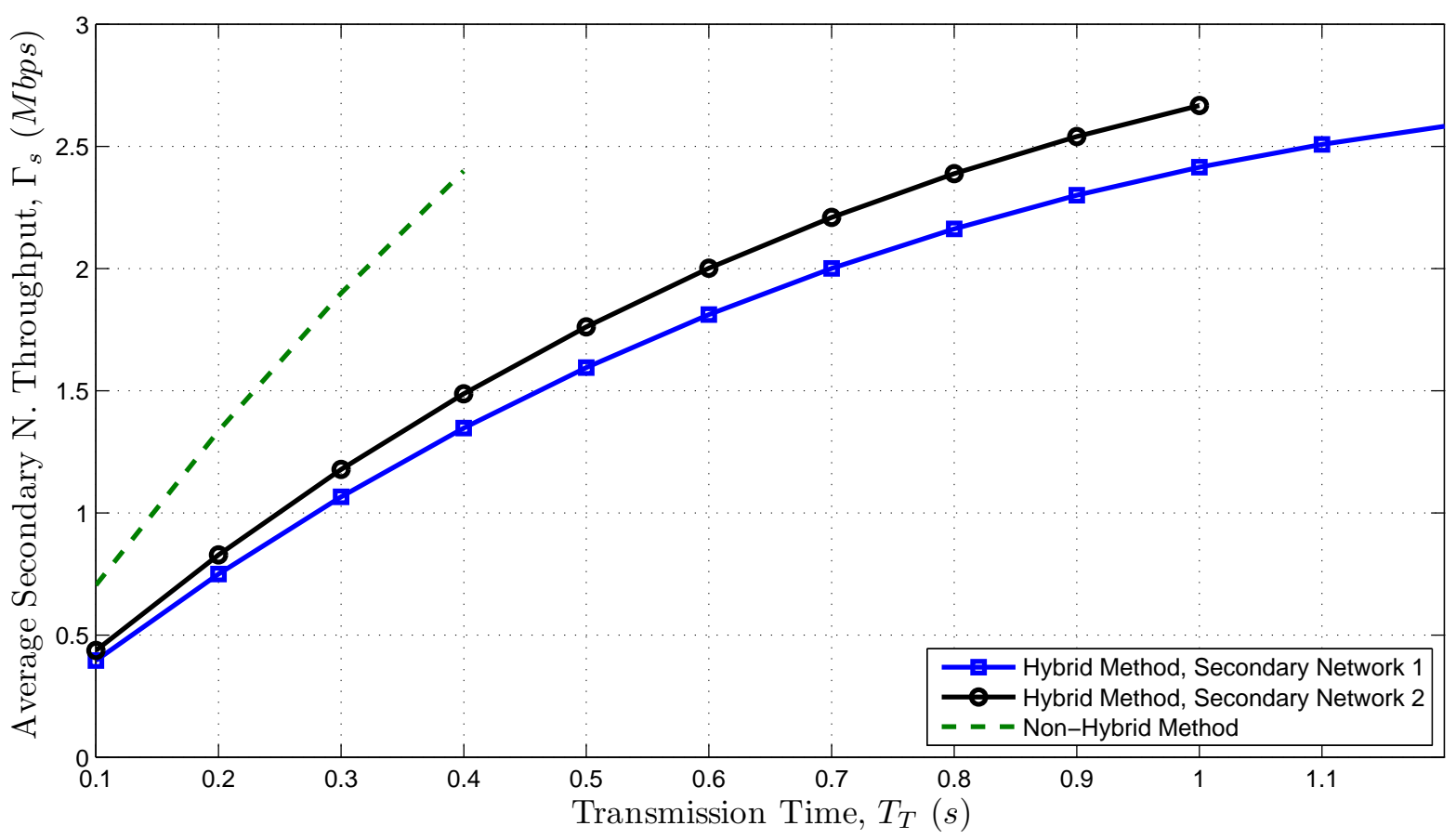

Figure 4.9: The average throughput of the secondary networks (theoretical results). 
the non-hybrid approach. As shown, the proposed method manages to reduce the power consumption by as low as $33 \%$ of the non-hybrid method for the secondary network 1 . Table 4.2 shows the simulation parameters used in this computer experiment.

As noted before, the proposed method aims to maximize the idle time $T_{I}$ during the idle state. While the maximization of $T_{I}$ is desirable to reduce the secondary network's power consumption, it is in contrast with the achievable throughput of the secondary network. In other words, larger idle time yields to less spectrum usage by the secondary network. Hence, there is a trade-off between the power consumption minimization and the throughput maximization.

In addition to the numerical analysis, we conduct simulation analysis to compare the performance of the application-specific method with the non-application-specific scheme. As shown in Fig. 4.11 and Fig. 4.12, the simulation results agree with the numerical results presented in this chapter.

Table 4.2: Simulation parameters for the throughput and power consumption analyses.

\begin{tabular}{|l|l||l|l|}
\hline Parameter & Value & Parameter & Value \\
\hline \hline$W$ & $200 \mathrm{KHz}$ & $G$ & 1 \\
\hline$E_{s 0}$ & $1(\mathrm{~mJ})$ & $P_{t 0}$ & $10(\mathrm{~mW})$ \\
\hline
\end{tabular}




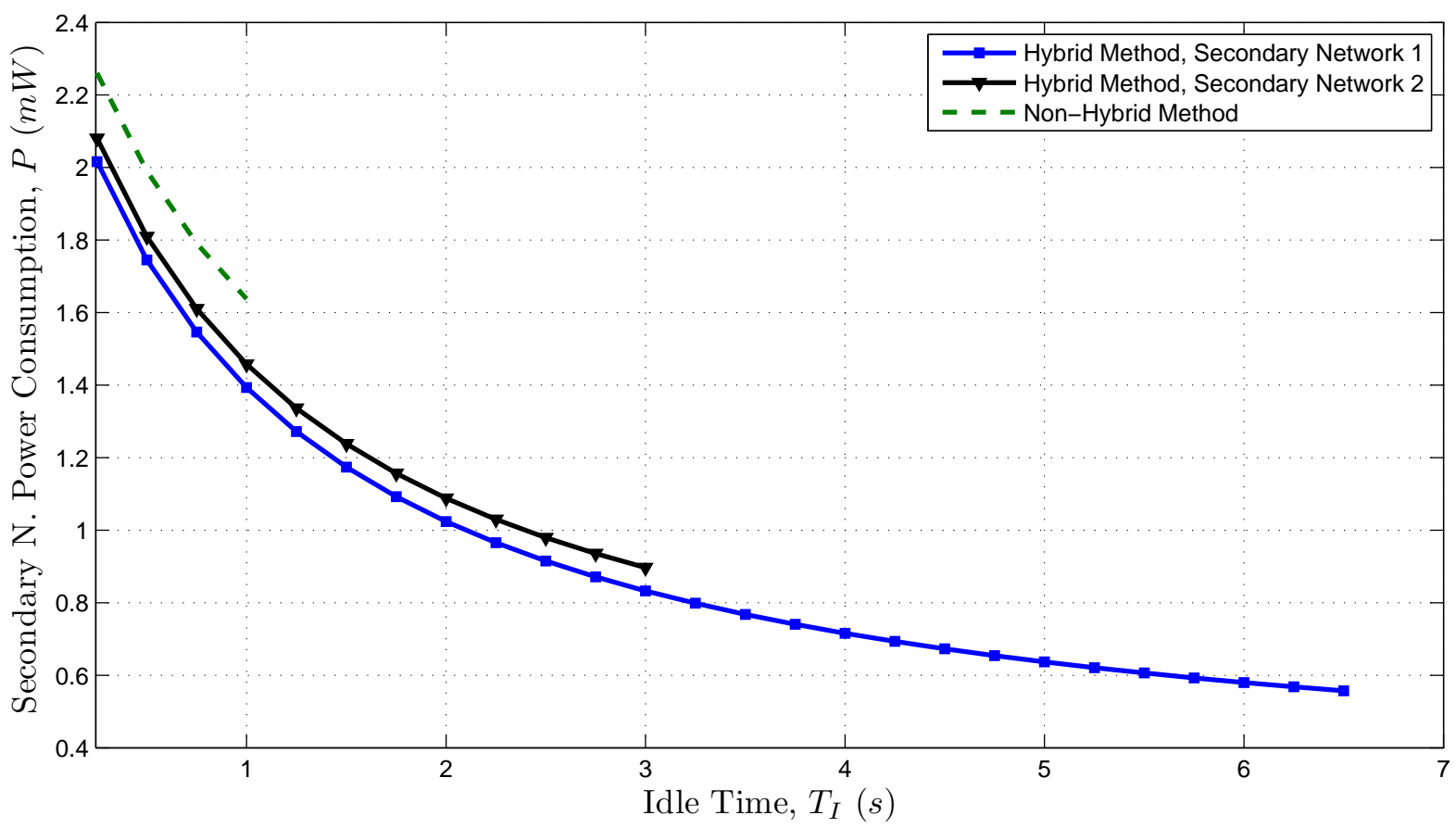

Figure 4.10: Total power consumption of the secondary networks (theoretical results).

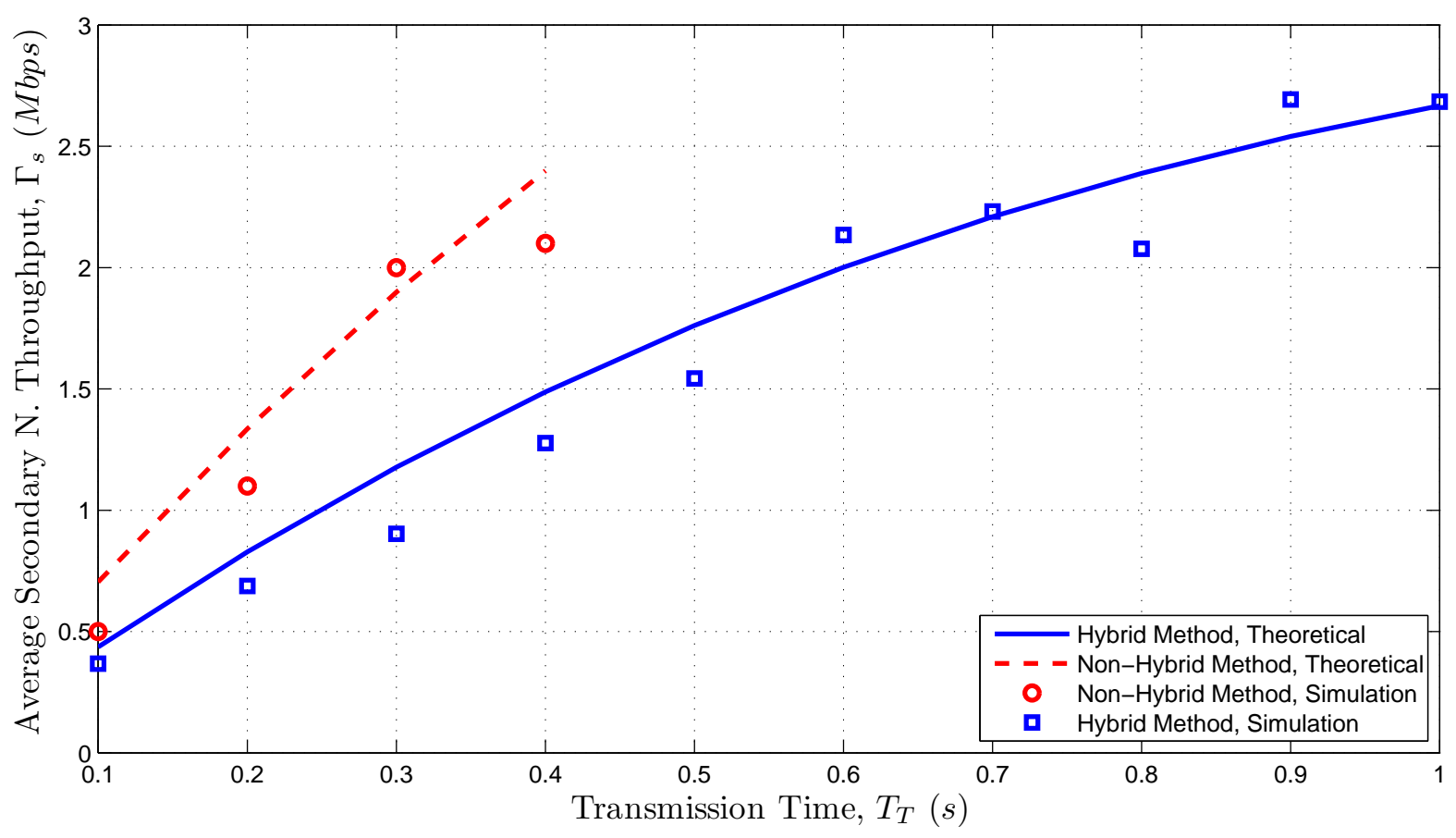

Figure 4.11: The secondary network's average throughput, simulation analysis. 


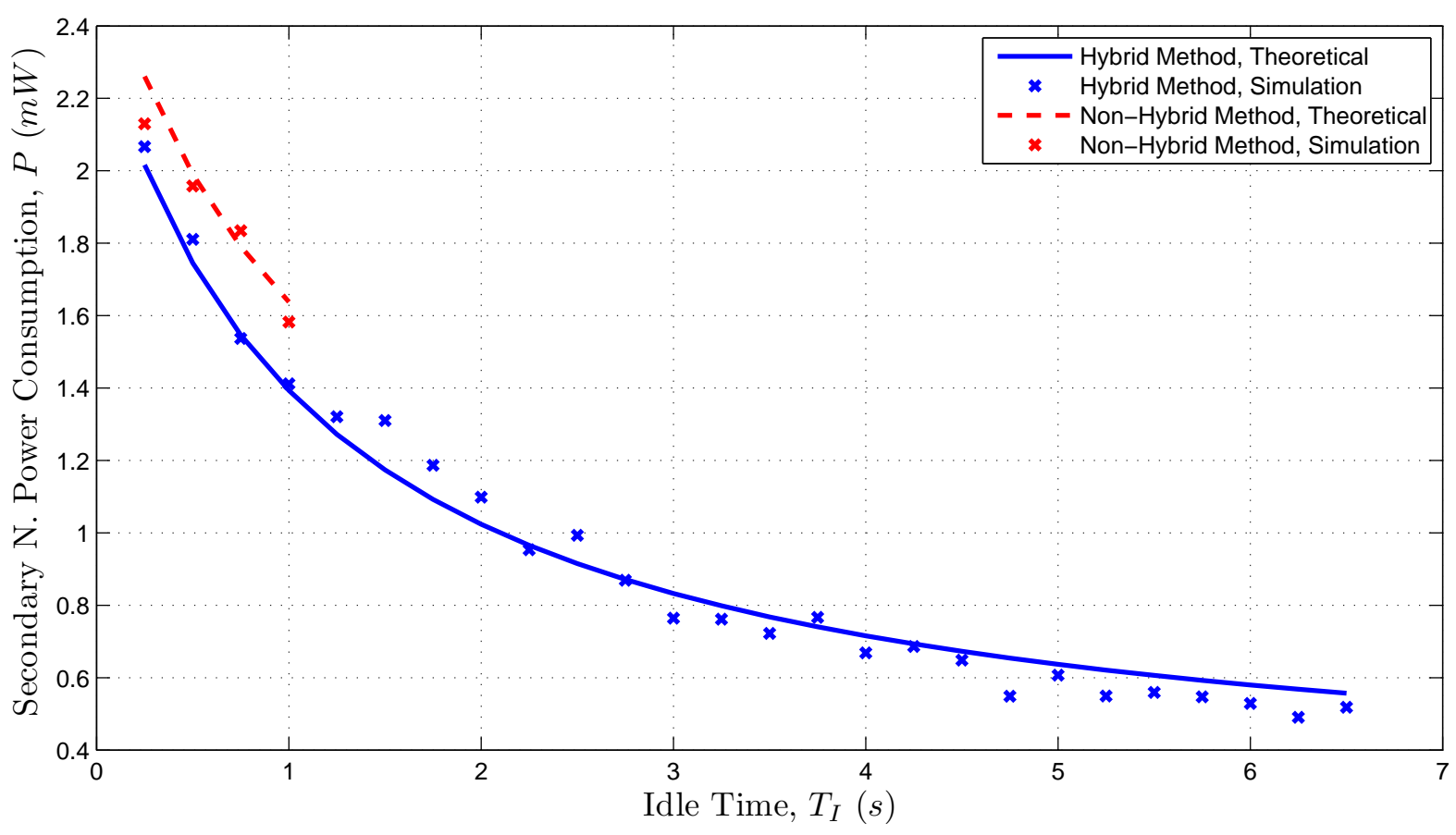

Figure 4.12: The secondary network's power consumption, simulation analysis.

\subsection{Summary}

In this chapter, we studied the problem of local sensing optimization in a cognitive sensor network. In contrast with the majority of existing work, where the sensing optimization is performed according to the properties of the primary network, we proposed an application-specific method that obtains the sensing period based on the properties of both primary and secondary networks. We then formulated two optimization problems to find the optimum sensing periods based on the secondary network's CTMC state.

In addition, the non-interfered system throughput and the total power consumption of the secondary network were derived by using a probability tree-diagram. We compared 
the performance of the proposed method with a non-application-specific scheme. In the following chapter, we will employ cooperative spectrum sensing to further improve the performance of the spectrum sensing. 


\section{Chapter 5}

\section{Energy-Aware Secondary User}

\section{Selection in Cooperative Spectrum}

\section{Sensing}

\subsection{Motivations}

Cooperative spectrum sensing is a promising scheme to tackle the hidden terminal problem ${ }^{1}$ by exploiting multi-user diversity $[24,25,70]$. The performance of the spectrum sensing is influenced by several factors, including sensing accuracy and energy consumption especially in an energy-constrained CR network, such as a CR-based sensor network.

\footnotetext{
${ }^{1}$ The hidden terminal problem occurs when several users accessing a channel while they cannot see or hear each other.
} 
In the literature, significant research effort has been made to tackle both issues individually. In [70], a partial spectrum sensing algorithm with decision result prediction and decision result modification is developed to reduce the cooperative spectrum sensing energy. A new channel management scheme for the CR sensor networks is proposed in [71], that increases the energy efficiency while not greatly disturbing PUs. According to the outcome of the channel sensing, the algorithm adaptively selects its operating mode among channel sensing, channel switching, and data transmission/reception. In [72], an optimal scheduling of sensor active time in sensor-aided CR networks is proposed to extend the network lifetime. The sensors are divided into a number of non-disjoint subsets such that only one subset of sensors is turned on at any time. Each subset is activated successively while other sensors sleep.

Another major focus of research is on the sensing accuracy - the precision in the task of the PU's signal detection - in a CR network. The sensing accuracy is evaluated by two metrics: the probability of detection $P_{d}$ and the probability of false alarm $P_{f}$. The larger the $P_{d}$, the better are PUs protected from SU interference. On the other hand, the smaller the $P_{f}$, the more efficiently the licensed channel can be used by SUs. A new cooperative wideband sensing scheme is proposed in [73] which exploits the spatial diversity among multiple SUs to improve the sensing accuracy. In [74], a large scale cognitive radio is considered, and a sequential sensing and fusion approach is presented to minimize the 
average sensing time by dividing the users into different sets according to their SNR parameters. Moreover, the sensing accuracy is evaluated in [75] for a cooperative sensing technique through multiuser cooperation.

However, the performance of the cooperate spectrum sensing relies on several uncertainties, such as the probability of error in the spectrum sensing task. A cognitive framework, therefore, should be aware of each SU's sensing accuracy, and give higher weight to more accurate sensing reports. Most of the existing work has overlooked the importance of the selection of the most eligible SUs for cooperate sensing.

In this chapter, we combine energy consumption and sensing accuracy in cooperative spectrum sensing and propose a novel method that is both energy-aware and accurate. First, we formulate and analytically solve an optimization problem to find the minimum number of required cooperative users, while satisfying the sensing task requirements. Then, we define a metric for sensing accuracy according to the most recent sensing reports, and employ a probability-based approach to identify the most eligible SUs. We also compare the network's lifetime for several sensing accuracy thresholds, and discuss the trade-off between the sensing accuracy and the network lifetime. 


\subsection{Cooperative Spectrum Sensing}

In the cooperative spectrum sensing, information from multiple SUs are incorporated to decide on the presence of the PU. In this approach, each SU performs local spectrum sensing independently and makes a binary decision on the presence or the absence of the PU. The local decision is then forwarded to a single entity called fusion centre (FC). Finally, the FC gathers all the local sensing results and makes a final conclusion to infer the presence or absence of the primary network. The final decision is then forwarded to all SUs, as shown in Fig. 5.1. We assume that the reporting channel is ideal in this work.

Several fusion rules have been discussed in the literature [76]. Here, we employ the $k$-out-of- $N$ fusion rule, where the spectrum is considered to be occupied by the PU when at least $k$ out of $N$ cooperative users declare that the $\mathrm{PU}$ is present. Therefore, the resulting hypothesis at the $\mathrm{FC}$ is given by

$$
\begin{array}{ll}
\sum_{i=1}^{N} D_{i}(n)<k & : H_{0} \\
\sum_{i=1}^{N} D_{i}(n) \geq k & : H_{1}
\end{array}
$$

In (5.1), $N$ is the number of cooperative SUs, and $D_{i}(n) \in\{0,1\}$ denotes the local sensing result of the $i^{\text {th }}$ secondary user at the $n^{\text {th }}$ sensing process. $D_{i}(n)=0$ corresponds to the absence of the $\mathrm{PU}$, and $D_{i}(n)=1$ corresponds to the presence of the PU. The 


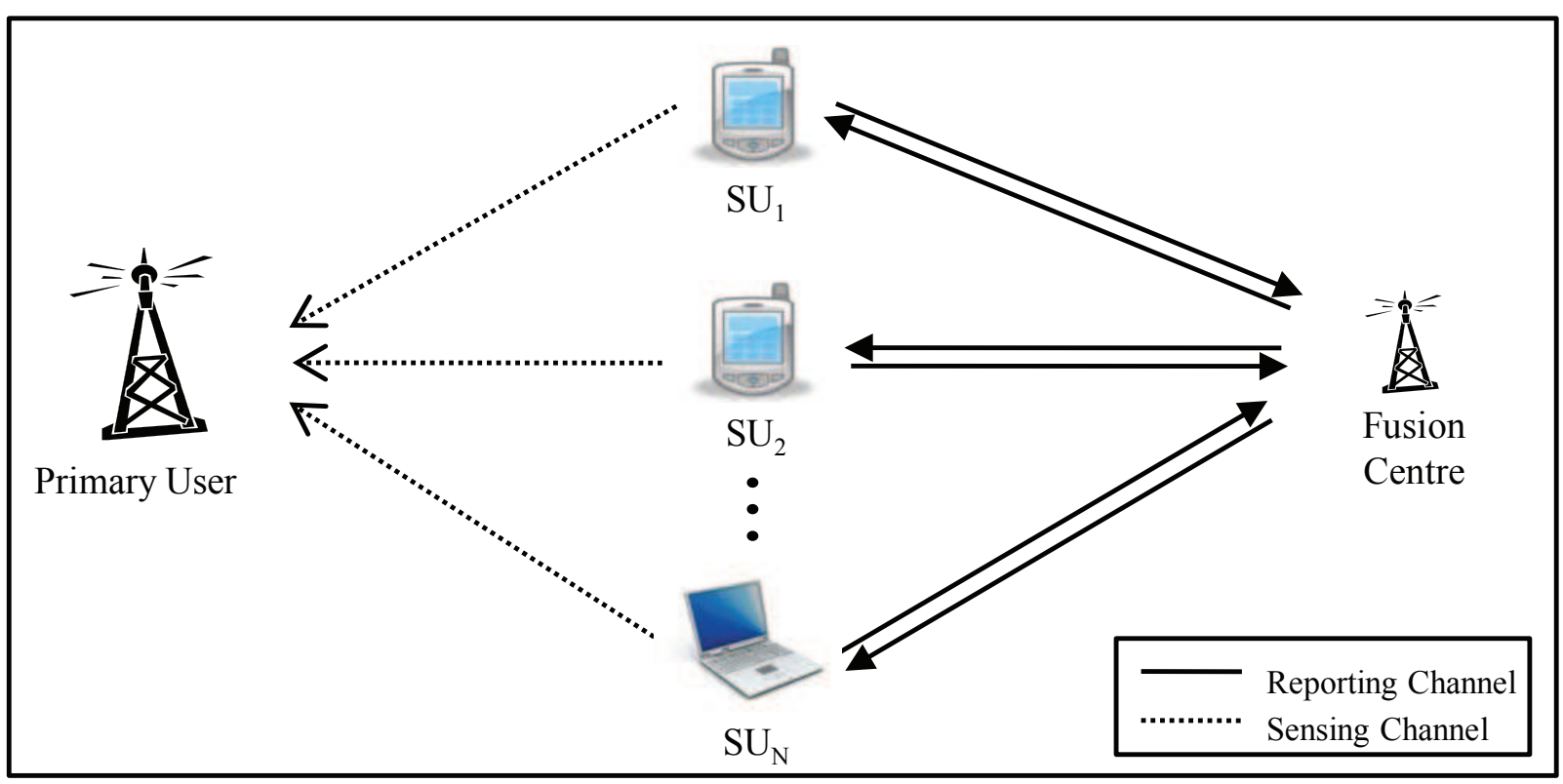

Figure 5.1: Cooperative spectrum sensing in a CSN.

probability of false alarm $P_{f}$ in (4.4) is considered for the case of no signal transmission $\left(H_{0}\right)$ and as such is identical for all secondary users [77]. In addition, we assume that, compared with the distance from any SU to the primary transmitter, the distance between any two SUs is small, so that the received signal at each SU experiences almost identical path loss. In the case of an AWGN environment, therefore, we can assume $\sigma_{s}^{2}$ is identical for all the secondary users. Consequently, $P_{d}$ in (4.5) will be identical for all SUs [78]. Therefore, the probability that at least $k$ secondary users declare that the PU is present 
given that the $\mathrm{PU}$ is absent, the global probability of false alarm $Q_{f}$, is given by

$$
Q_{f}=\sum_{i=k}^{N}\left(\begin{array}{c}
N \\
i
\end{array}\right)\left(P_{f} \cdot P_{a c t}\right)^{i}\left(1-P_{f} \cdot P_{a c t}\right)^{N-i}
$$

where $P_{a c t}$ is the probability that the secondary network is active. Moreover, the probability that at least $k$ secondary users declare that the spectrum is occupied by the PU, given that the spectrum is occupied by the $\mathrm{PU}$, the global probability of detection $Q_{d}$, is obtained by

$$
Q_{d}=\sum_{i=k}^{N}\left(\begin{array}{c}
N \\
i
\end{array}\right)\left(P_{d} \cdot P_{a c t}\right)^{i}\left(1-P_{d} \cdot P_{a c t}\right)^{N-i}
$$

The AND rule will be an special case of the k-out-of-N rule by letting $k=N$. The global probability of false alarm and the global probability of detection, therefore, are obtained by plugging in $k=N$ in (5.2) and (5.3), as follows:

$$
\begin{aligned}
& Q_{f}^{A N D}=\left(P_{f} \cdot P_{a c t}\right)^{N} \\
& Q_{d}^{A N D}=\left(P_{d} \cdot P_{a c t}\right)^{N} .
\end{aligned}
$$

Similarly, the OR rule will be an special case of the k-out-of-N rule by letting $k=1$ 
in (5.2) and (5.3) as follows:

$$
\begin{gathered}
Q_{f}^{O R}=1-\left(1-P_{f} \cdot P_{a c t}\right)^{N} . \\
Q_{d}^{O R}=1-\left(1-P_{d} \cdot P_{a c t}\right)^{N} .
\end{gathered}
$$

\subsection{Energy-Aware Cognitive User Selection}

The proposed method aims to improve the performance of the spectrum sensing in two directions. First, it selects a subset of SUs to perform the sensing/reporting task. The minimum number of required secondary users $\left(N^{*}\right)$ in the subset is derived to minimize the network's energy consumption, while satisfying the spectrum sensing require-

ments. Second, the proposed method identifies the most eligible SUs to perform the sensing/reporting task.

\subsubsection{Minimum Number of Required Cognitive Users in the Subset}

As noted before, it is expected to minimize the number of cooperative users in the subset, so that the overall energy consumption of the secondary network is reduced. However, the performance of the cooperative sensing will also degrade with fewer SUs (less multi- 
user diversity); therefore, there is an optimum size for the subset. We formulate the cooperative sensing problem as follows:

\section{Minimize: $N^{*}$}

Subject to: $Q_{f} \leq \gamma_{f}$, and $Q_{d} \geq \gamma_{d}$

where $N^{*}$ is the minimum number of required cooperative SUs, and $\gamma_{f}$ and $\gamma_{d}$ are the system thresholds set for the global probability of false alarm and the global probability of detection respectively. In order to solve the above optimization problem, we first derive $\epsilon$ from (4.5) as follows:

$$
\epsilon=\left(\sigma_{s}^{2}+\sigma_{n}^{2}\right)\left(Q^{-1}\left(P_{d}\right) \sqrt{2 M}+M\right)
$$

where $Q^{-1}(\cdot)$ is the inverse Q-function. Next, $\epsilon$ is plugged into (4.4) as follows:

$$
P_{f}=Q\left(\frac{Q^{-1}\left(P_{d}\right) \cdot\left(\sigma_{s}^{2}+\sigma_{n}^{2}\right) \sqrt{2 M}+M \sigma_{s}^{2}}{\sigma_{n}^{2} \sqrt{2 M}}\right)
$$

Note that $Q_{f}$ in (5.2) follows a binomial distribution. Therefore, we can use the 
incomplete beta function ${ }^{2} I$ to express $P_{f}$ as a function of $Q_{f}$ as follows:

$$
Q_{f}=1-\operatorname{Pr}(i<k)=1-\operatorname{Pr}(i \leq k-1)=1-I_{1-P_{f} \cdot P_{a c t}}(N-k+1, k)
$$

where $I_{z}(a, b)=\frac{B(z ; a, b)}{B(a, b)}$ is the regularized incomplete beta function, $B(z ; a, b)=\int_{0}^{z} t^{a-1}(1-$ $t)^{b-1} d t$ is the incomplete beta function, and $B(a, b)=\int_{0}^{1} t^{a-1}(1-t)^{b-1} d t$ is the beta function. Using the inverse of the regularized incomplete beta function, $P_{f}$ can be written as follows:

$$
P_{f}=\frac{1}{P_{a c t}}\left(1-I_{1-Q_{f}}^{-1}(N-k+1, k)\right)
$$

where $I_{1-Q_{f}}^{-1}(\cdot)$ is the inverse of the regularized incomplete beta function.

In a similar way, $P_{d}$ can be derived from $Q_{d}$ in (5.3) as follows:

$$
P_{d}=\frac{1}{P_{a c t}}\left(1-I_{1-Q_{d}}^{-1}(N-k+1, k)\right)
$$

Note that for a given $k$ and $N$ in the k-out-of- $N$ fusion rule, $I$ and $I^{-1}$ are monotonically increasing functions. Therefore, the optimization problem constraint $Q_{f} \leq \gamma_{f}$ can be written as

$$
P_{f}=\frac{1}{P_{a c t}}\left(1-I_{1-Q_{f}}^{-1}(N-k+1, k)\right) \leq \varsigma_{f}
$$

\footnotetext{
${ }^{2}$ The incomplete beta function is the cumulative distribution function of a random variable $x$ with a binomial distribution, where the probability of success is $p$ and the sample size is $n$. Hence, $\operatorname{Pr}[x \leq k]=$ $I_{1-p}(n-k, k+1)$ [79], page 1112 .
} 
where $\varsigma_{f}=\frac{1}{P_{a c t}}\left(1-I_{1-\gamma_{f}}^{-1}(N-k+1, k)\right)$.

Similarly, $Q_{d} \geq \gamma_{d}$ can be written as

$$
P_{d}=\frac{1}{P_{a c t}}\left(1-I_{1-Q_{d}}^{-1}(N-k+1, k)\right) \geq \varsigma_{d}
$$

where $\varsigma_{d}=\frac{1}{P_{a c t}}\left(1-I_{1-\gamma_{d}}^{-1}(N-k+1, k)\right)$.

As shown in (5.14), $P_{d} \geq \varsigma_{d}$. Also, $Q^{-1}(\cdot)$ is a monotonically decreasing function, therefore, we get

$$
Q^{-1}\left(P_{d}\right) \leq Q^{-1}\left(\varsigma_{d}\right)
$$

In addition, $Q(\cdot)$ is a monotonically decreasing function as well. Hence, (5.9) and (5.14) yield

$$
P_{f} \geq Q\left(\frac{Q^{-1}\left(\varsigma_{d}\right) \cdot\left(\sigma_{s}^{2}+\sigma_{n}^{2}\right) \sqrt{2 M}+M \sigma_{s}^{2}}{\sigma_{n}^{2} \sqrt{2 M}}\right) .
$$

Therefore, based on (5.13) and (5.16), the minimum number of required cooperative users $N^{*}$ will be the minimum solution of the following inequality,

$$
Q\left(\frac{Q^{-1}\left(\varsigma_{d}\right) \cdot\left(\sigma_{s}^{2}+\sigma_{n}^{2}\right) \sqrt{2 M}+M \sigma_{s}^{2}}{\sigma_{n}^{2} \sqrt{2 M}}\right) \leq \varsigma_{f}
$$

Finally, an exhaustive search for $N$ from 1 to the first value that satisfies (5.17) is 
employed to find $N^{*}$.

\subsubsection{Identification of the Most Eligible SUs}

In this section, we assume that the cognitive users achieve different sensing accuracy levels. The probability of detection $P_{d}$, therefore, would be different for each secondary user. However, $P_{f}$ is identical for all the SUs because it is calculated for the case of no signal transmission $\left(H_{0}\right)$, as discussed in the previous section. The operation of the proposed method is divided into time units called epochs. Each epoch is further divided into several sub-units called rounds, where each round lasts for $T_{P}^{*}$ seconds (the optimum sensing period). Fig. 5.2 depicts a timeline for the proposed method. As shown, the first $W$ rounds in each epoch are assigned to the setup phase, and the remaining $L$ rounds are reserved for the operational phase. The secondary network will be active for a fraction of $L$ rounds within the operation phase with the probability of $P_{a c t}$.

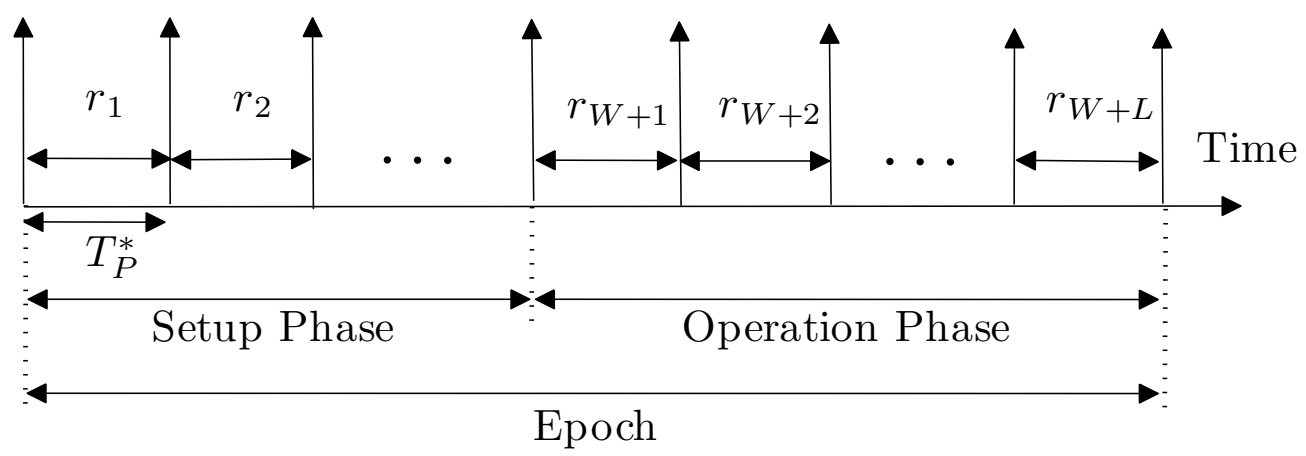

Figure 5.2: The proposed method's timeline. 
As mentioned earlier, $D_{i}(n) \in\{0,1\}$ denotes the result of local sensing performed by the $i^{\text {th }}$ secondary user during the $n^{\text {th }}$ sensing task. Also, let $F(n) \in\{0,1\}$ denote the result of FC's decision that is obtained by fusing all the collected reports at the FC within the $n^{\text {th }}$ round. At the end of the setup phase, we define $\Delta_{i}(n)$ as the total number of differences between the $i^{\text {th }}$ secondary user's local sensing and the FC's global decision within the last $W$ rounds as follows:

$$
\Delta_{i}(n)=\sum_{j=0}^{W-1}\left|D_{i}(n-j)-F(n-j)\right|
$$

We assume that the FC does not know the performance of the SUs a priori. Therefore, during the setup phase, all SUs are required to perform the spectrum sensing/reporting task. The most eligible SUs are then selected based on the SUs performance within this phase. The smaller the $\Delta_{i}(n)$ is, the more accurate the local sensing will be and vice versa. Therefore, a probability-based method is proposed that selects a subset of SUs that has the most accurate sensing results. Let us define $\eta_{i}$ as a weighting factor assigned to the $i^{\text {th }} \mathrm{SU}\left(u_{i}\right)$, where the probability to select $u_{i}$ is proportional to $\eta_{i}$. Since the proposed method aims to reduce the amount of inaccurate sensing results, the probability that a user $u_{x}$ with low $\Delta_{x}(n)$ is selected must be higher than the probability that a user $u_{y}$ 
with a high $\Delta_{y}(n)$ is selected. Hence, we define $\eta_{i}(n)$ as

$$
\eta_{i}(n)=\frac{1}{1+r \Delta_{i}(n)}
$$

where $r$ is a positive real number such that $r>1$. The probability to select $u_{i}$ within the $n^{\text {th }}$ sensing task, therefore, is defined as the weighting factor assigned to $u_{i}$ over the summation of all weighting factors, as follows:

$$
P_{i}(n)=\frac{\eta_{i}(n)}{\sum_{j=1}^{N} \eta_{j}(n)} .
$$

We note that the energy consumption is one of the biggest concerns in a CR-based sensor network. Therefore, it is not prudent to choose the active users without considering the remaining energy of each SU. In particular, there might be circumstances where a particular SU has a high sensing accuracy due to its location (for instance, being close to the PU). In such cases, if the energy level is not considered, the same SU would be repeatedly selected, and its energy will be drained quickly. As a result, in addition to the sensing accuracy, we consider the ratio of each SU's energy to the initial secondary network's energy within each round, and revise the $\eta_{i}(n)$ function defined in (5.19) as follows:

$$
\eta_{i}(n)=\frac{1}{1+r \Delta_{i}(n)+g \frac{e_{0}}{e_{i}(n)}}
$$


where $g$ is a positive real number such that $g>1, e_{0}$ is the initial energy of the cognitive users, and $e_{i}(n)$ is the remaining energy of $u_{i}$ at the $n^{\text {th }}$ sensing process. We assume that each time a $\mathrm{SU}$ is selected, its energy level will be drained by $e_{u}$, where $e_{u}$ is the energy consumed to perform a single sensing/reporting task. Note that (5.21) reduces to (5.19) for $g=0$. Fig. 5.3 depicts the proposed method's flowchart, where $r$ denotes the current round, $c_{-}$setup and $c_{-}$op are the current number of rounds in the setup and operation phases respectively, and epoch is the number of rounds in an epoch. As shown, when $\bmod (r-1$, epoch $) \leq W-1$, the algorithm goes into the setup phase. Otherwise, it transits to the operation phase. The Check-Dead function is invoked at the beginning of both phases to check if (1) any SU is dead, and (2) the secondary network is dead. We consider an SU to be dead when its energy level is less than $e_{u}$. Moreover, the CNS is pronounced dead if the number of alive SUs is less than $N^{*}$. Fig. 5.4 presents a subroutine to check the number of dead SUs, called the Check-Dead function.

Let $x$ denote the ratio of the accuracy multiplier to the energy level multiplier, i.e., $x=\frac{r}{g}$. Therefore, (5.21) is re-written as,

$$
\eta_{i}^{x}(n)=\frac{1}{1+g\left(x \Delta_{i}(n)+\frac{e_{0}}{e_{i}(n)}\right)} .
$$

We consider (5.22) and study two cases highlighting the emphasis on the accuracy or 


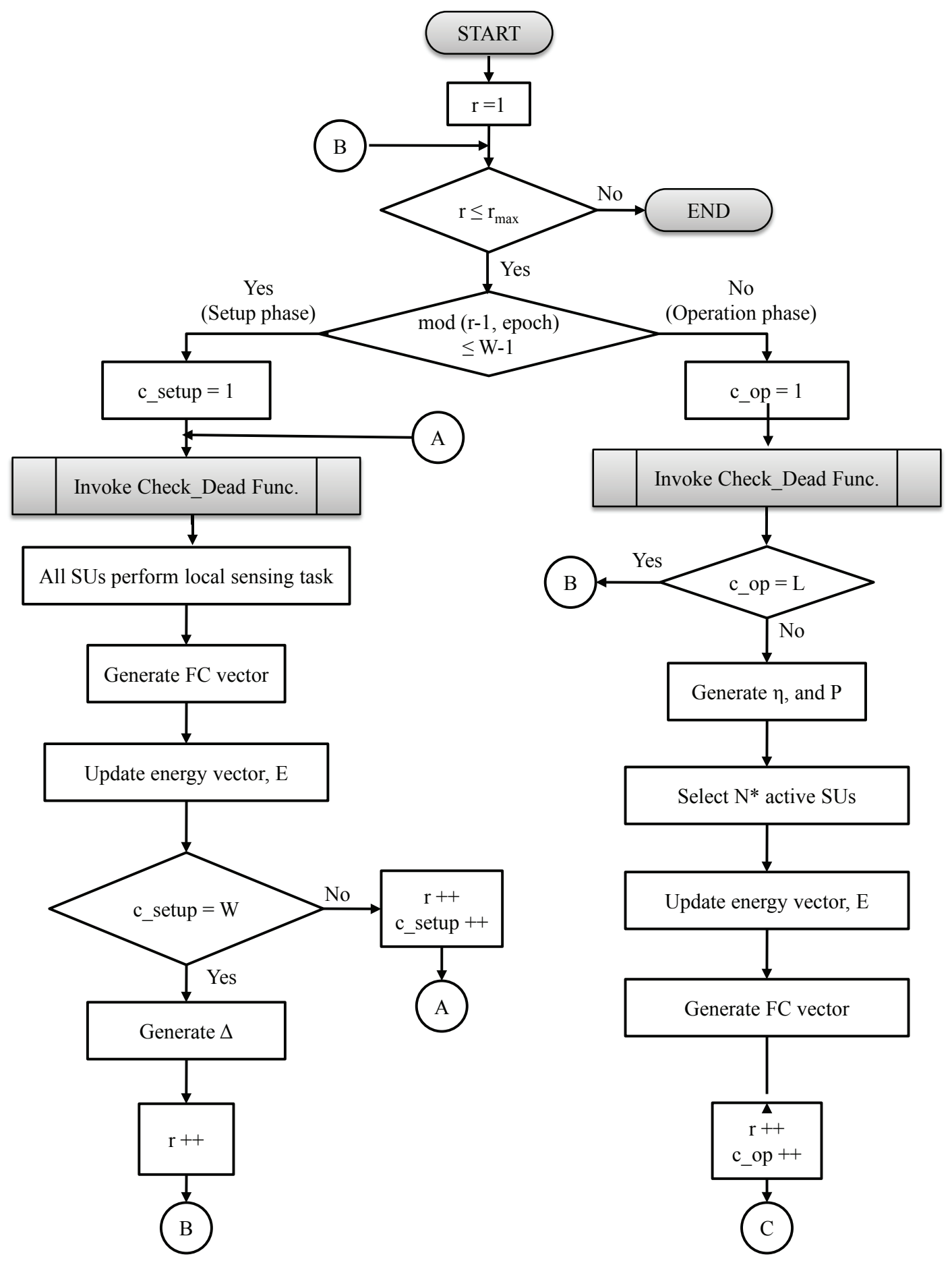

Figure 5.3: The proposed method's flowchart; $r$ denotes the current round, $c \_s e t u p$ and $c_{-}$op are the current number of rounds in the setup and operation phases respectively, and epoch is the number of rounds in an epoch. 


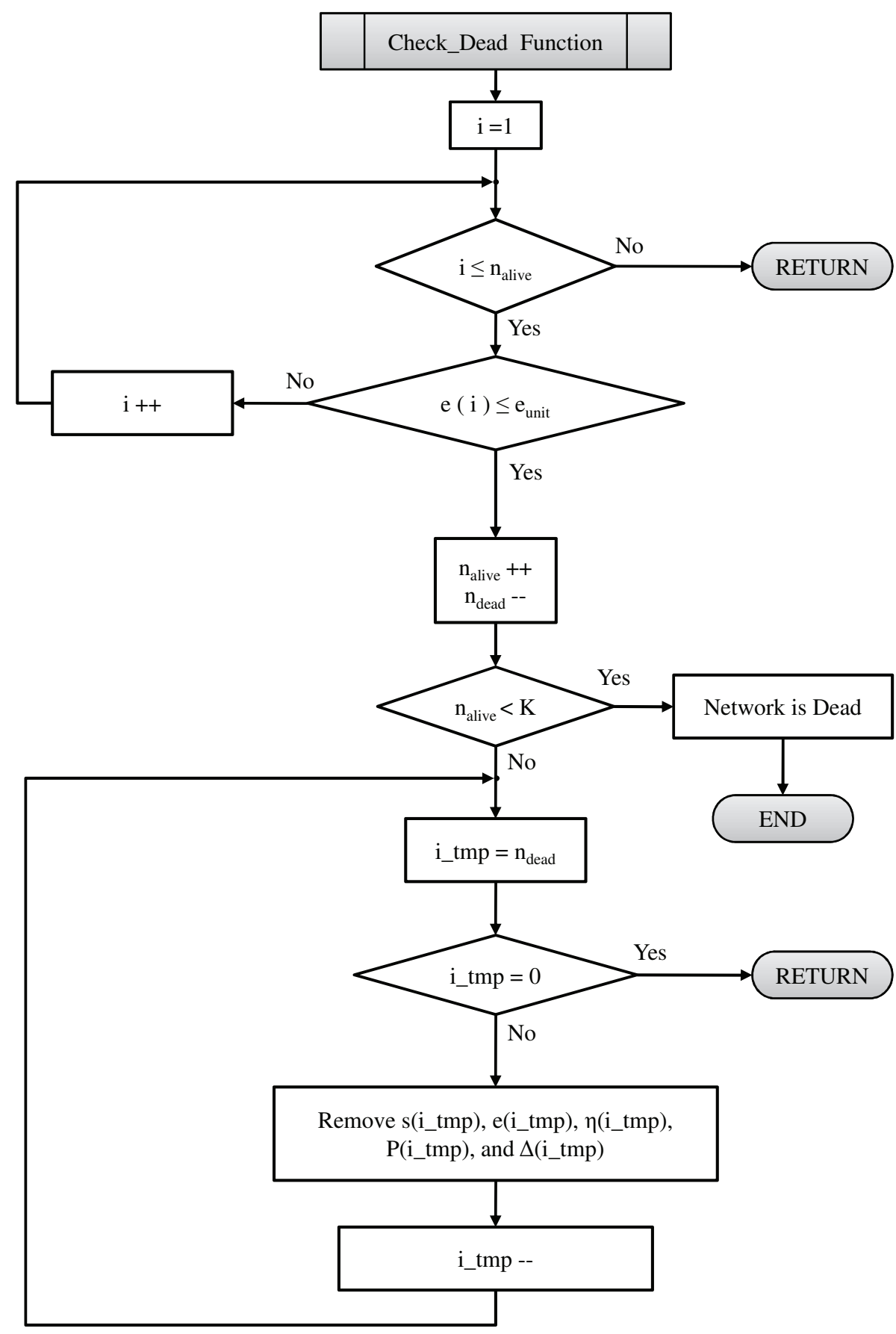

Figure 5.4: Check-Dead: a subroutine to check the number of dead SUs. $n_{\text {dead }}$ and $n_{\text {alive }}$ are the number of dead and alive SUs respectively. 
on the energy level of the secondary network, as follows:

- Case-I $(x>1)$ : in this case, the sensing accuracy is emphasized by making the accuracy multiplier $(r)$ greater than the energy multiplier $(g)$.

- Case-II $(x \leq 1)$ : in this case, the emphasis is on the energy level by making the accuracy multiplier $(r)$ smaller than the energy multiplier $(g)$.

During the operation phase, based on $P_{i}(n)$, the most $N^{*}$ secondary users will be selected at each round to perform the sensing/reporting tasks. The rest of the SUs, however, will be inactive to save the secondary network's energy consumption.

The probability to select the $i^{\text {th }}$ secondary user within the $n^{\text {th }}$ sensing task defined in (5.20) can be revised to identify the most eligible secondary users with a reasonable accuracy level, as follows:

$$
P_{i}^{\prime}(n)= \begin{cases}\frac{\eta_{i}(n)}{\sum_{j=1}^{N} \eta_{j}(n)} & \text {, if } \Delta_{i}(n) \leq \Delta_{t h} \\ 0 & \text {,if } \Delta_{i}(n)>\Delta_{t h}\end{cases}
$$

where $\Delta_{t h}$ is a predefined system threshold set for the sensing accuracy. Hence, the secondary users that have a $\Delta$ function greater than $\Delta_{t h}$ in each round are not eligible to be selected, i.e., the probability to select those secondary users is zero. 


\subsubsection{Evaluation Benchmarks}

In the following, we set two benchmarks for the performance evaluation of the proposed method:

- Sensing accuracy benchmark $\left(P_{0}\right)$ is defined as the probability to select an SU that has $\Delta_{i}(n)=0$, averaged over the simulation time as follows:

$$
P_{0}=E\left\{\sum_{i=1}^{N} \operatorname{Pr}\left[\Delta_{i}(n)=0\right]\right\}
$$

where $E\{$.$\} is the average function. Note that the greater the P_{0}$ is, the more accurate the cooperative sensing will be.

- Energy benchmark $\left(P_{l e}\right)$ is defined as the probability to select an $\mathrm{SU}$ which has the lowest level of energy at each round (averaged over the simulation time). Hence, it would be desirable to have the lowest value for the $P_{l e}$.

\subsection{Numerical and Simulation Results}

In this section, we conduct several computer experiments to evaluate the performance of the proposed method and to study the effects of adjustable parameters on the proposed method. In the first place, the effect of system thresholds $\varsigma_{f}$ and $\varsigma_{d}$ on the minimum 
number of required cooperative users $\left(N^{*}\right)$ is studied. Fig. $5.5 \operatorname{depicts} N^{*}$ as $\varsigma_{f}$ varies; as depicted, $N^{*}$ is reduced when the system requirement on $P_{f}$ is more relaxed. Moreover, as shown in Fig. 5.5, the minimum number of required cooperative users is increased when the SNR is decreased $\left(S N R=10 \log \frac{\sigma_{s}^{2}}{\sigma_{n}^{2}}\right)$.

In the second place, Fig. 5.6 shows the minimum number of required cooperative users as a function of $\varsigma_{d}$. As depicted, $N^{*}$ is increased when the system requirement on the probability of detection is more tight, i.e., when $\varsigma_{d}$ is larger. Moreover, as expected, a larger number of cooperative users $\left(N^{*}\right)$ are needed to perform the cooperative sensing task when the system has a smaller SNR.

Here, we study the effect of the number of rounds within the setup phase $(W)$ on the sensing accuracy. As shown in Fig. 5.7, $P_{0}$ is greater for greater values of $W$. In other words, the sensing accuracy will be improved by larger values of $W$.

In addition, the weighting function $\eta_{i}^{x}(n)$ defined in (5.22) is applied to investigate the effect of emphasizing the accuracy versus emphasizing the remaining level of energy. We use an arbitrary $x>1$ value to emphasize the accuracy, and an arbitrary $x<1$ value to emphasize the energy. The simulation is run for $1 \leq g \leq 50$, and the corresponding $P_{0}$ and $P_{l e}$ values are recorded. Table 5.1 summarizes the results of this simulation. As depicted, the emphasis on the sensing accuracy improves the sensing accuracy benchmark by $22 \%$. Similarly, emphasis on the energy improves the energy benchmark by as much 


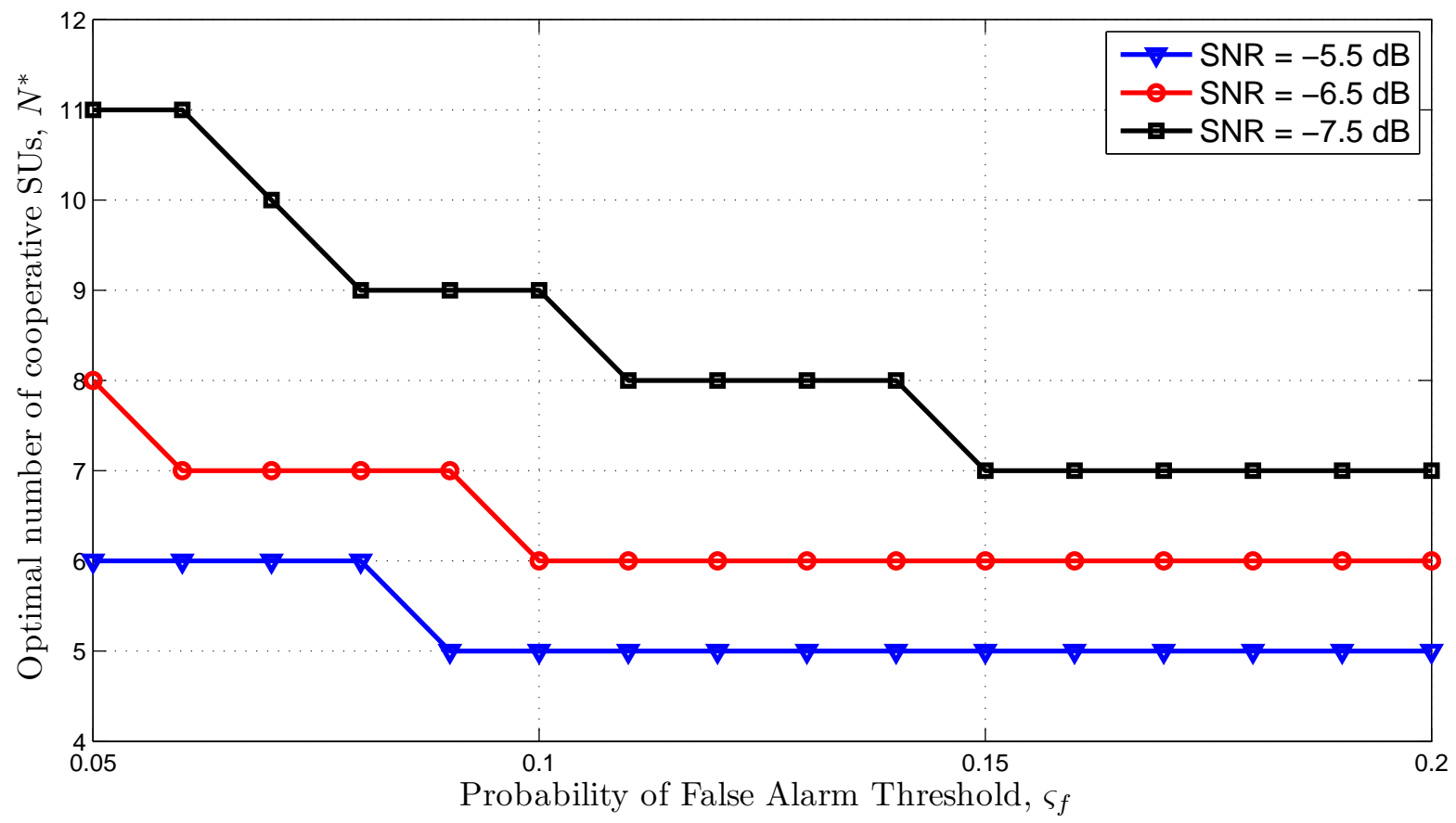

Figure 5.5: Minimum number of required cooperative users as a function of $\varsigma_{f},\left(\varsigma_{d}=0.85\right)$.

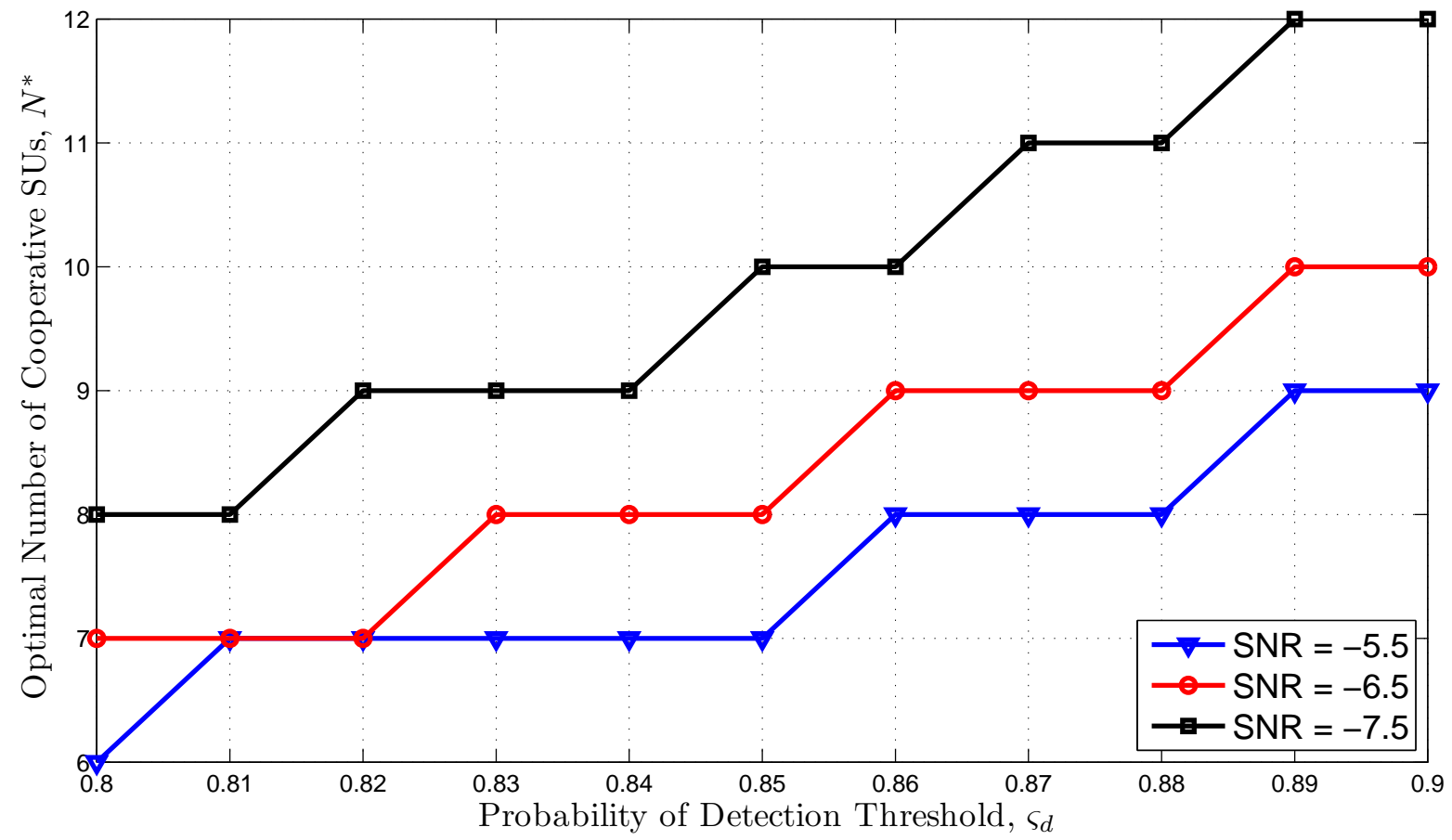

Figure 5.6: Minimum number of required cooperative users as a function of $\varsigma_{d},\left(\varsigma_{f}=0.2\right)$. 


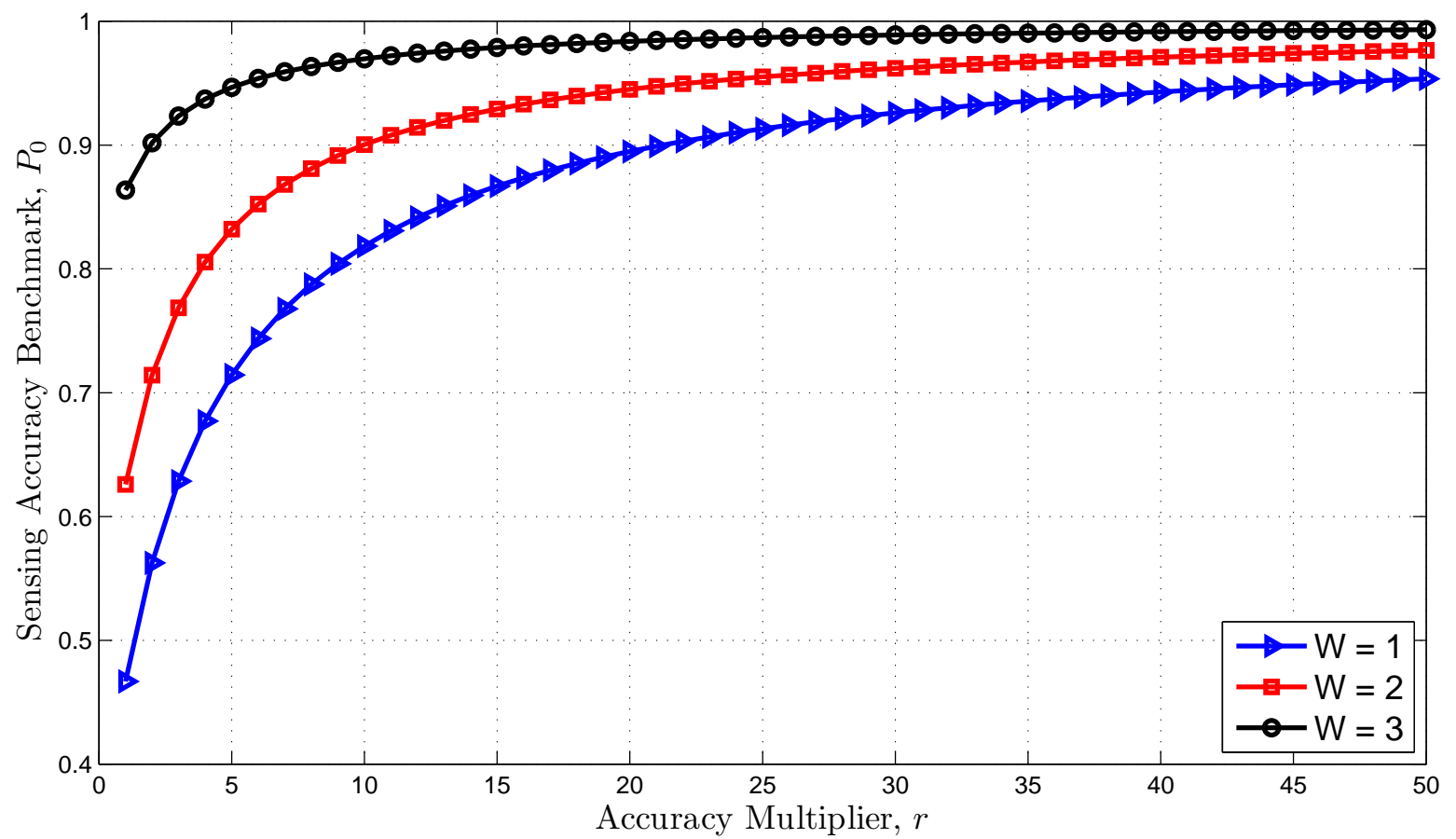

Figure 5.7: The effect of the number of rounds within the setup phase $(W)$.

as $20 \%$.

We also examine the following extreme cases: in Case-A, the remaining level of energy is not considered in the selection of the SUs, i.e., $g=0$ in (5.21). Similarly, in Case-B, the accuracy of the spectrum sensing is not considered, i.e., $r=0$ in (5.21). Fig. 5.8 depicts

Table 5.1: Emphasizing the sensing accuracy vs. the secondary network's energy.

\begin{tabular}{|l||l|l|}
\hline & $\begin{array}{l}\text { Max. of the sensing accuracy } \\
\text { benchmark, } \max \left\{P_{0}\right\}\end{array}$ & $\begin{array}{l}\text { Min. of the energy bench- } \\
\text { mark, } \min \left\{P_{l e}\right\}\end{array}$ \\
\hline $\begin{array}{l}\text { Emphasis on the accuracy, } \\
(x=5)\end{array}$ & 0.93 & 0.29 \\
\hline $\begin{array}{l}\text { Emphasis on the energy, }(x= \\
0.2)\end{array}$ & 0.76 & 0.24 \\
\hline \hline Comparison & $\begin{array}{l}\text { Emphasis on the sensing accu- } \\
\text { racy increases } P_{0} \text { by } 22 \%\end{array}$ & $\begin{array}{l}\text { Emphasis on the network's } \\
\text { energy reduces } P_{l e} \text { by } 20 \%\end{array}$ \\
\hline
\end{tabular}


$P_{0}$ and $P_{l e}$ benchmarks for the Case-A and the Case-B. As shown, the sensing accuracy is improved when the accuracy multiplier $(r)$ increases. However, the performance of the proposed method in regard to $P_{l e}$ deteriorates. In addition, the performance of the proposed method with respect to $P_{l e}$ is improved by increasing the energy multiplier $(g)$ in Case-B, while the accuracy $\left(P_{0}\right)$ does not vary with the change in the energy multiplier.

Therefore, among the Case- $\mathrm{A}$ and the Case- $\mathrm{B}$, it is concluded that the algorithm provides a better performance with respect to the sensing accuracy in Case-A, and it has a better performance in regard to $P_{l e}$ in Case-B. As shown in Fig. 5.8, the accuracy benchmark $P_{0}$ is increased by as much as $39 \%$ in Case-A. Additionally, the energy benchmark $P_{l e}$ is reduced by as low as $76 \%$ in Case-B.

\subsubsection{The Secondary Network's Lifetime}

In another study, we consider a CSN with $N=9$ secondary users. The minimum number of required SUs to perform the sensing/reporting task is assumed to be $N^{*}=5$. We use the $P_{i}^{\prime}(n)$ definition in (5.23), and apply the k-out-of-N rule for $k=3$. The number of rounds in the setup and operational phases are set to be $W=10$ and $L=90$ respectively. Furthermore, the initial energy of each $\mathrm{SU}$ is assumed to be $e_{0}=75(\mathrm{~J})$, and the energy consumed to perform each sensing/reporting task is set to be $e_{u}=0.1(\mathrm{~J})$. Table 5.2 summarizes the above parameters. Based on the sensing accuracy, SUs are also grouped 

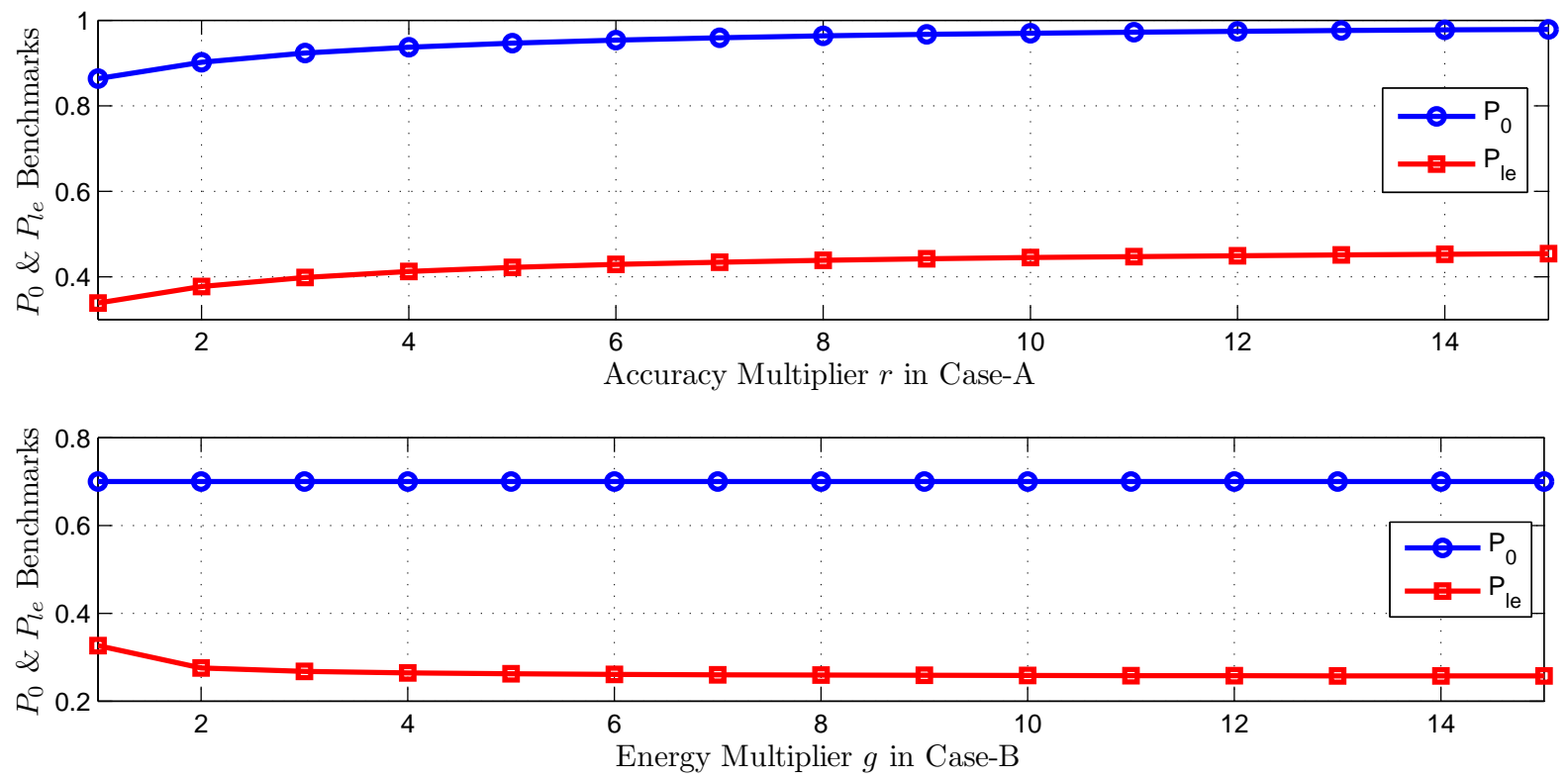

Figure 5.8: The accuracy and energy benchmarks in Case-A and Case-B.

into three categories, as depicted in Table 5.3.

The secondary network's lifetime is compared at different sensing accuracy thresholds.

The simulation is run and the number of SUs is recorded until the number of alive SUs is greater than $N^{*}$; then we consider the network to be dead. As depicted in Fig. 5.9, the secondary network's lifetime is shortened by enforcing a better sensing accuracy (an smaller accuracy threshold, $\Delta_{t h}$ ). In other words, there is a trade-off between the secondary network's lifetime and the sensing accuracy.

Table 5.2: Simulation parameters.

\begin{tabular}{|l|l||l|l|}
\hline \hline Parameter & Value & Parameter & Value \\
\hline$N$ & 9 & $N^{*}$ & 5 \\
\hline$W$ & 10 & $L$ & 90 \\
\hline$e_{0}$ & $75(\mathrm{~J})$ & $e_{u}$ & $0.1(\mathrm{~J})$ \\
\hline
\end{tabular}


Table 5.3: Classification of the secondary users into three categories.

\begin{tabular}{|l|c|c|c|}
\hline \hline & $S U_{1}-S U_{3}$ & $S U_{4}-S U_{6}$ & $S U_{7}-S U_{9}$ \\
\hline$P_{d}$ & 0.9 & 0.85 & 0.8 \\
\hline Sensing Accuracy & Good & Average & Poor \\
\hline
\end{tabular}

Note that by setting a smaller value for the sensing accuracy threshold, SUs that have better sensing accuracy would be selected more often; therefore, the energy of such users will be deployed sooner. This is also depicted in Fig. 5.10. As shown, in the case of $\Delta_{t h}=0$, SUs that have higher sensing accuracy are selected with higher frequency compared to those that have lower sensing accuracy. For instance, $S U_{1}, S U_{2}$, and $S U_{3}$ which have the highest sensing accuracies (see table 5.3) are selected more often, as depicted in Fig. 5.10. However, in case of $\Delta_{t h}=4$, SUs are selected with almost the same frequency.

\subsubsection{The Fusion Rule's Effect}

In this section, several computer simulations are conducted to examine the effects of different fusion rules on the proposed method's performance. First, the average $P_{d}$ of the secondary users is varied, and the simulation is run for 10,000 rounds. Fig. 5.11 depicts the probability of interference $\left(P_{I}\right)$ for the OR Rule, the AND Rule, and the Majority Rule. As shown, the OR rule provides the smallest probability of interference among the mentioned fusion rules. In the OR rule, even if one SU reports a local sensing decision 


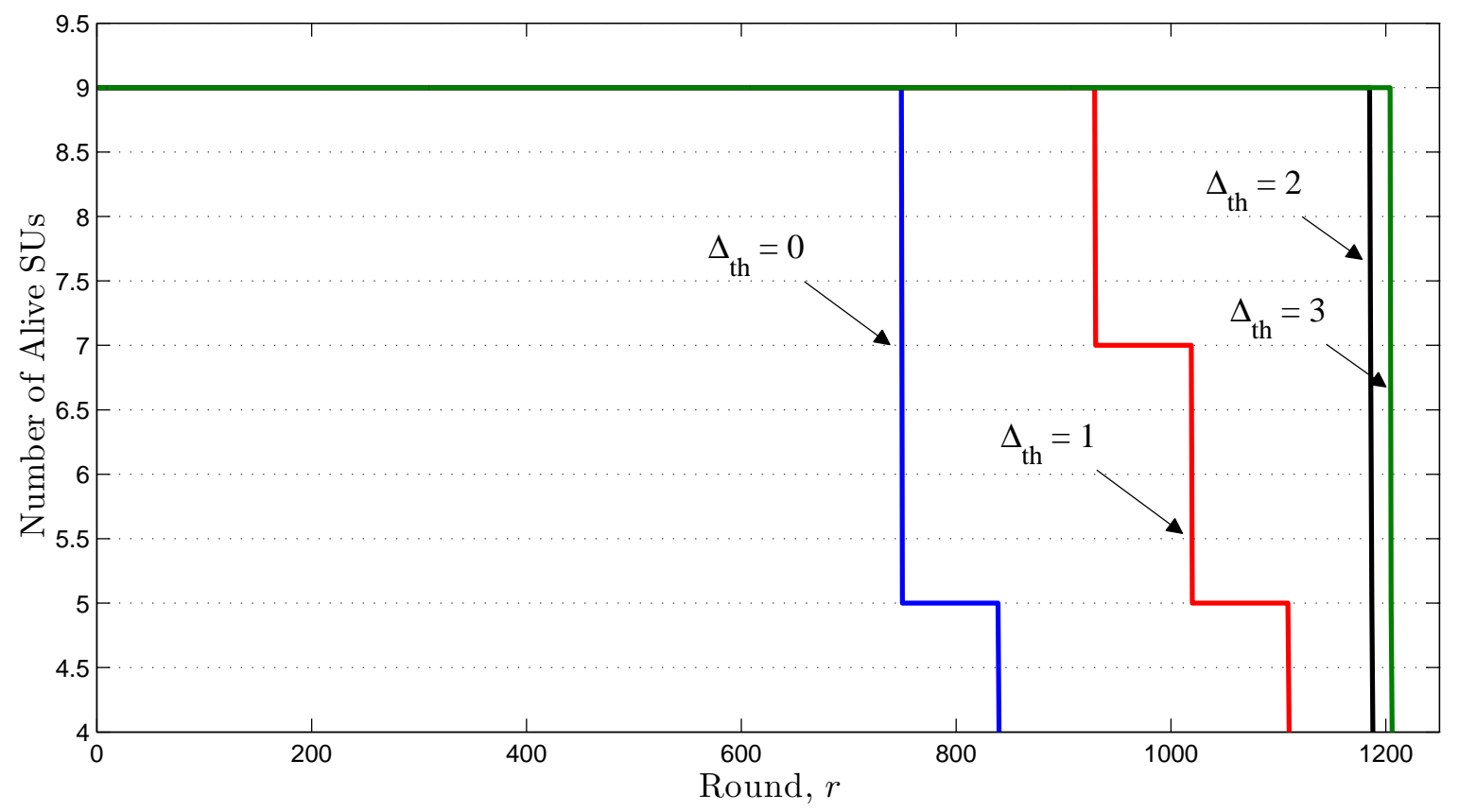

Figure 5.9: The secondary network's lifetime at different values of $\Delta_{t h}$.

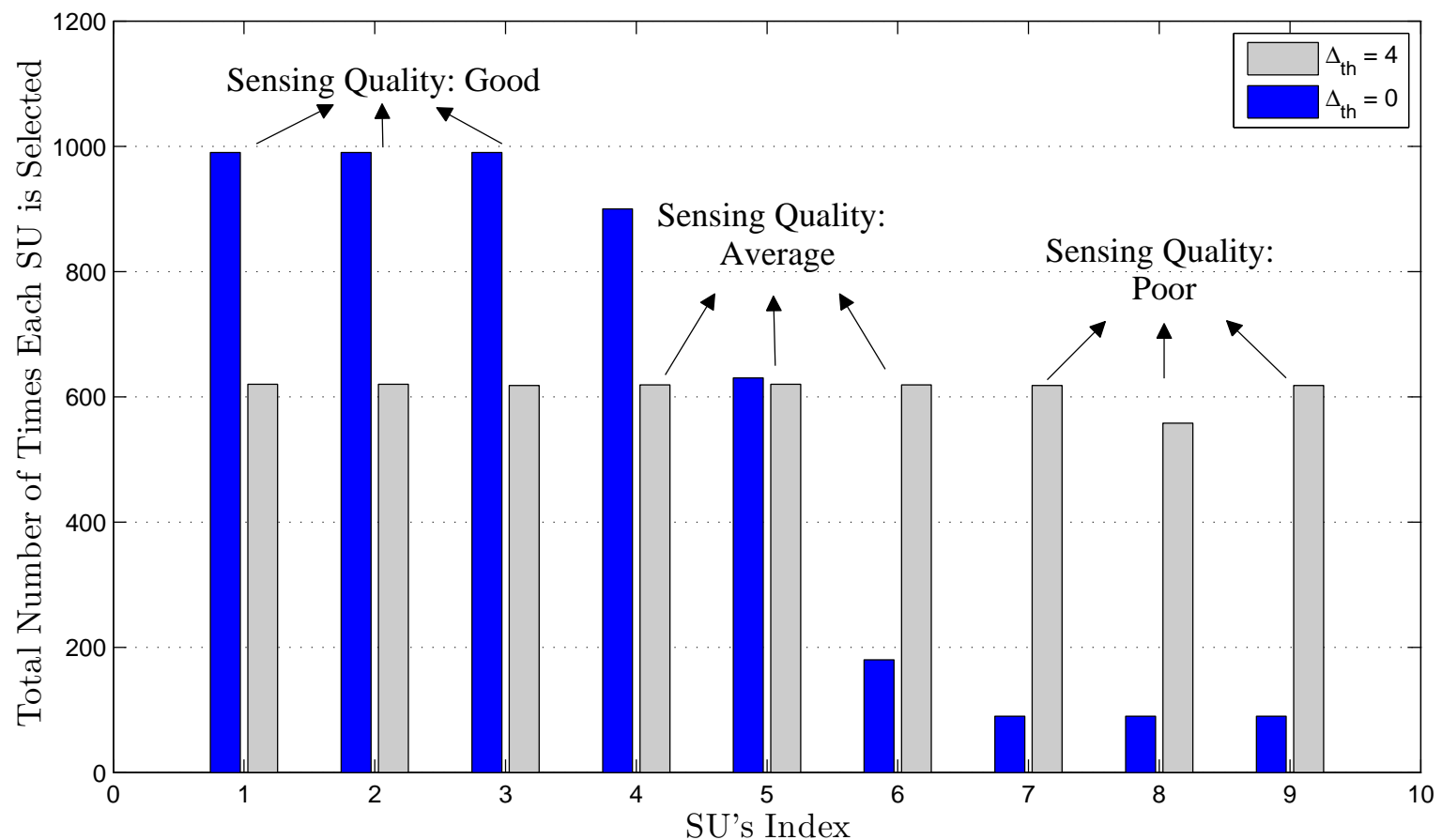

Figure 5.10: Total number of times each secondary user is selected at $\Delta_{t h}=0$ and $\Delta_{t h}=4$. 
of "1", the FC will conclude that the PU is operating in the spectrum. Hence, the PU is fully protected from the SUs interference unless all the cooperative SUs report a "0". On the other hand, in the AND rule, even if one SU sends a local decision of "0", the FC will conclude that the PU is not operating in the spectrum. Therefore, the AND rule has the largest probability of interference. The probability of false alarm $P_{f}$ is set to be 0.05. The acceptable range of $P_{d}$ (the operation region) is set to $P_{d} \geq 0.8$.

We also vary the average $P_{f}$ of the cognitive users and run the simulation for 10,000 rounds. The probability of missed opportunities $\left(P_{m}\right)$ is then examined among the mentioned rules. As shown in Fig. 5.12, the OR rule has the worst performance with the largest probability of missed opportunities. In the OR rule, even one local sensing of "1" makes the FC conclude that the PU is present. Therefore, if the sensing report is not correct, a missed opportunity will occur. On the other hand, the AND rule has the best performance with the smallest $P_{m}$. This is due to the fact that unless all SUs report a "1", the AND rule will conclude that the PU is not present. Hence, the number of missed opportunities is minimal. For this computer simulation, the probability of detection $P_{d}$ is set to be 0.8 , and the acceptable range of $P_{f}$ (the operation region) is set to $P_{f} \leq 0.2$.

As depicted in Fig. 5.11 and Fig. 5.12, the OR rule has the best performance with respect to the probability of interference. However, it has the worst performance from the missed opportunities point of view. On the other hand, the AND rule has the best 


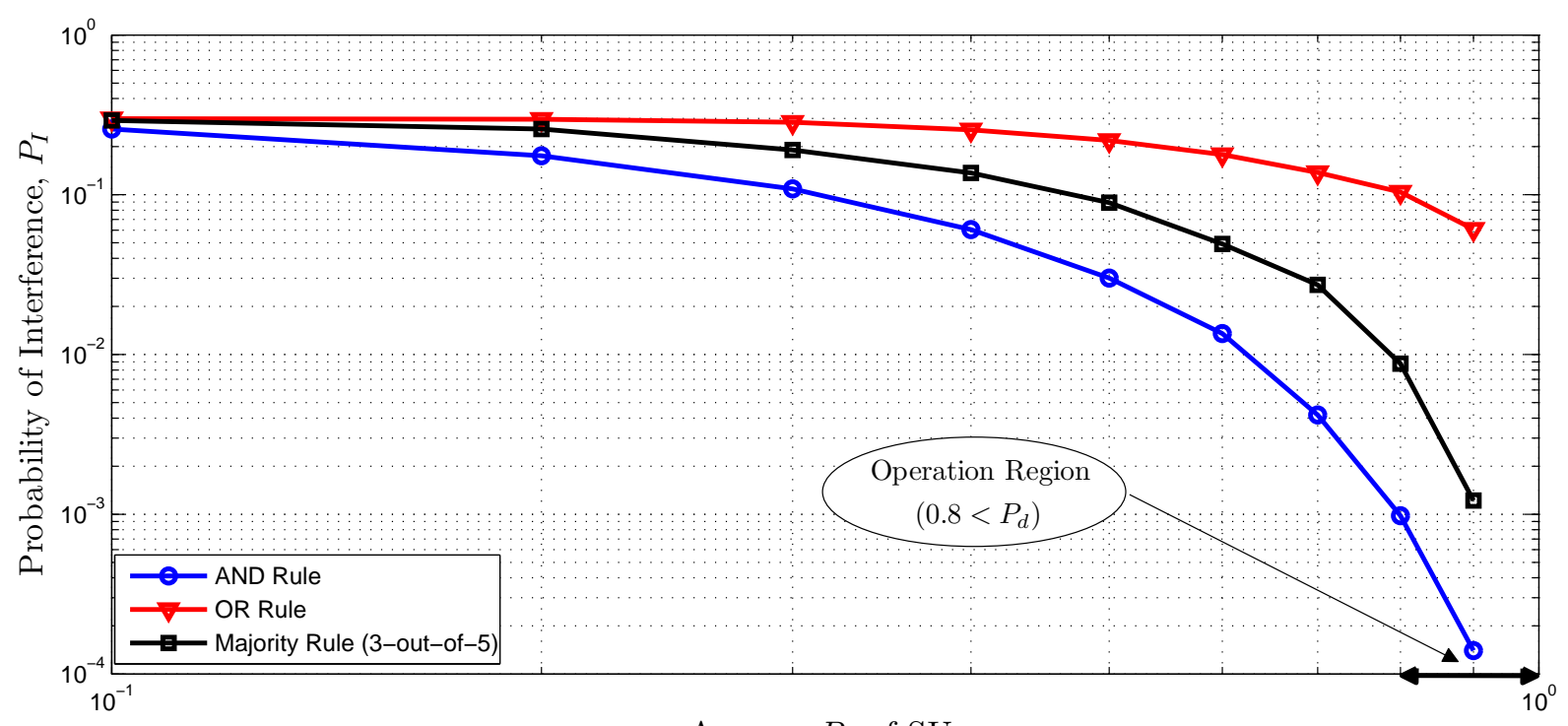

Average $P_{d}$ of SUs

Figure 5.11: The probability of interference $P_{I}$ for different fusion rules.

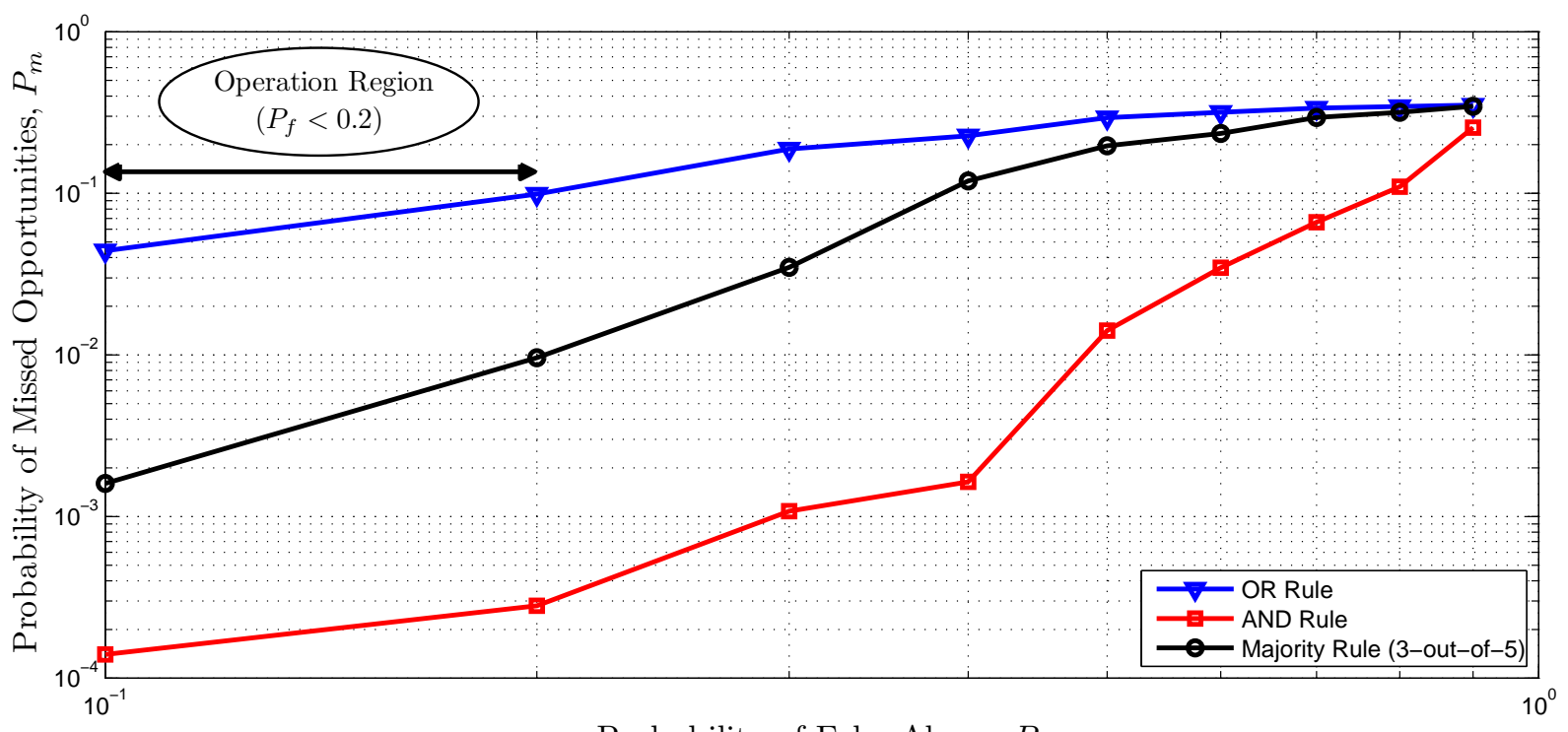

Probability of False Alarm, $P_{f}$

Figure 5.12: The probability of missed opportunities $P_{m}$ for different fusion rules. 
performance with respect to the missed opportunities, but it has the worst performance with respect to interference. However, as shown in Fig. 5.11 and Fig. 5.12, the Majority rule provides a close result to the best options (that is the OR rule in the interference study, and the AND rule in the missed opportunities study). Therefore, we conclude that the Majority rule has the best overall performance among the examined rules. This can be also verified by studying the probability of error $\left(P_{e}\right)$, as performed in Fig. 5.13. As shown, the Majority rule has the smallest probability of error. Similarly, the probability of false alarm $P_{f}$ is set to be 0.05 , and the operation region is set to $P_{d} \geq 0.8$.

In another computer simulation, we consider a CSN with $N=20$ secondary users, and assume that the minimum required number of cooperative users is $N^{*}=10$. We run the simulation for 10,000 rounds and record the probability of error for different values of $k$ in the k-out-of- $\mathrm{N}$ rule to examine the effect of other fusion rules. As depicted in Fig. 5.14, the Majority rule has the best performance with the minimum $P_{e}$. The OR rule and the AND rule, however, have the worst performances as discussed before.

In addition, we perform numerical analysis for rounds $1 \leq r \leq 10$ to further enlighten the proposed method's approach for spectrum sensing. We consider a CSN with five SUs $(N=5)$, where the minimum number of required SUs is assumed to be $N^{*}=2$. Moreover, the initial energy of each $\mathrm{SU}$ is set to $e_{0}=1(\mathrm{~J})$, and the energy consumed to perform each sensing/reporting task is $e_{u}=0.1(\mathrm{~J})$. The duration of the setup phase is 


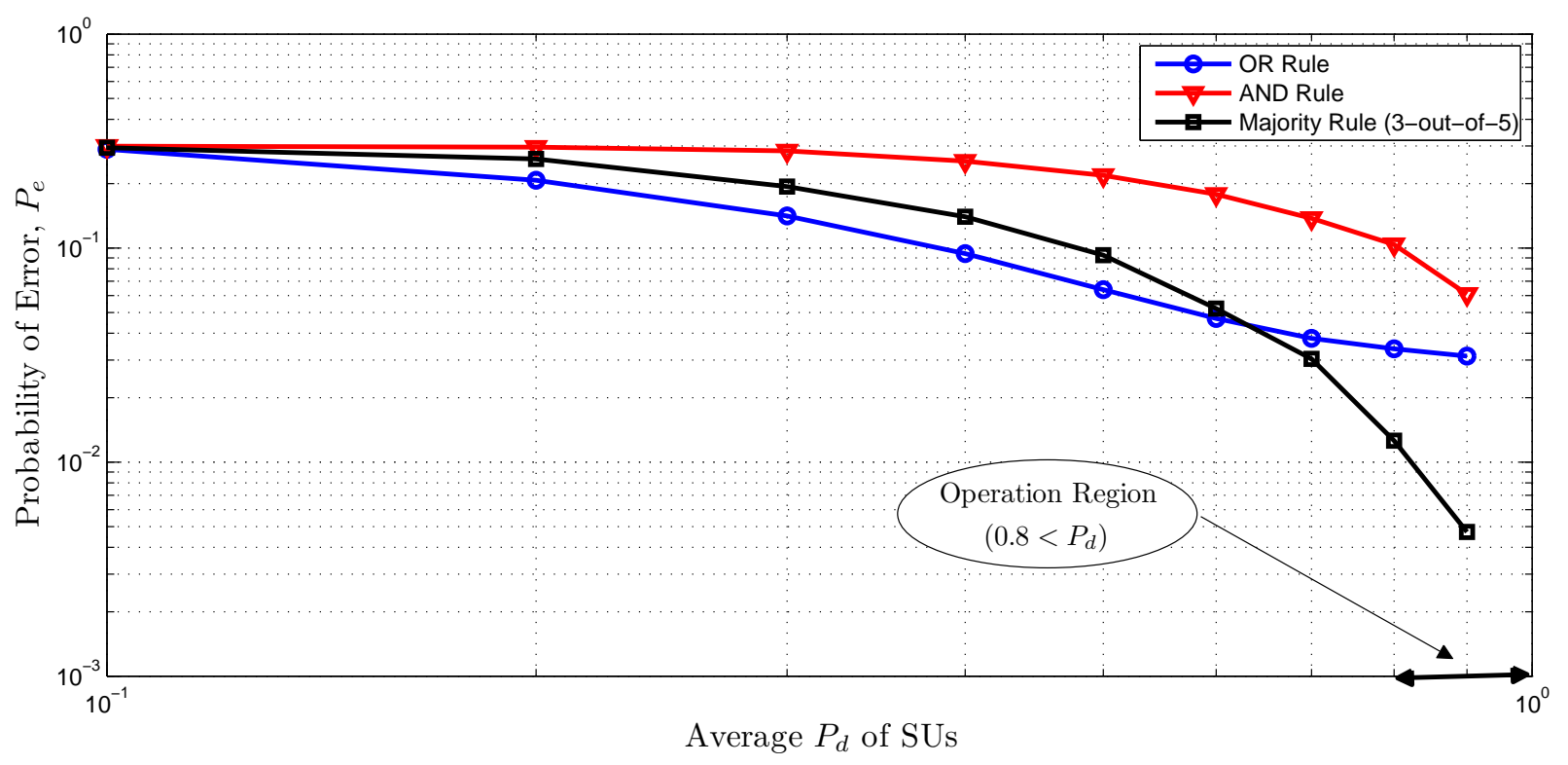

Figure 5.13: The probability of error $P_{e}$ for different fusion rules.

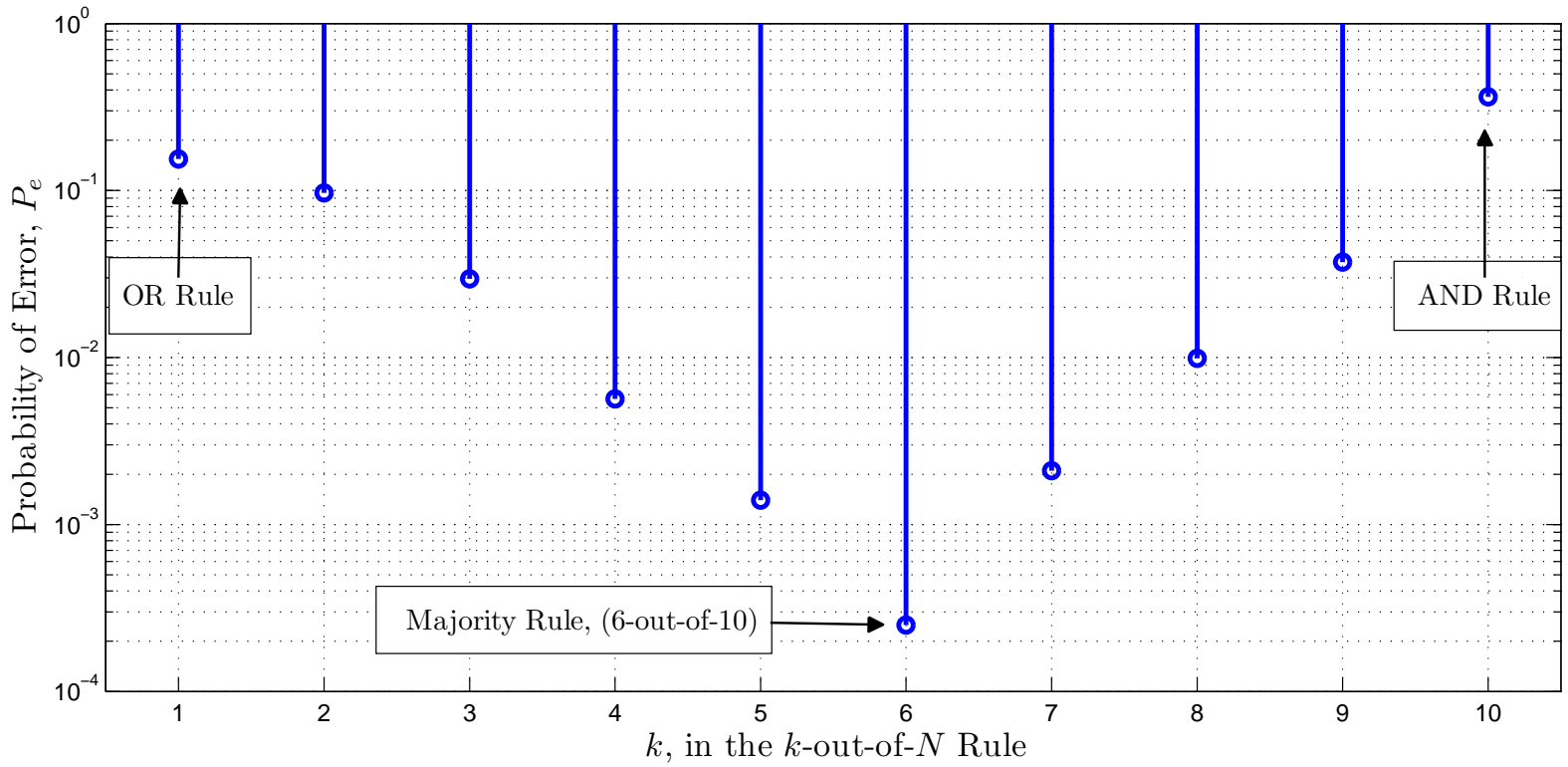

Figure 5.14: The probability of error $P_{e}$ for different values of $k$ in the k-out-of-N rule $(1 \leq k \leq 10)$. The minimum required number of cooperative users is considered to be $N^{*}=10$. 
set to be $W=2$ rounds, and the number of rounds within the operation phase is $L=4$ rounds. As shown in Fig. 5.15, rounds $\left\{r_{1}, r_{2}, r_{7}, r_{8}\right\}$ are assigned to the setup phase, and rounds $\left\{r_{3}, r_{4}, r_{5}, r_{6}, r_{9}, r_{10}\right\}$ are assigned to the operation phase. The PU's ON-OFF pattern is also depicted in Fig. 5.15 as [ $\left.\begin{array}{llllllllll}1 & 1 & 0 & 0 & 0 & 1 & 1 & 0 & 1 & 1\end{array}\right]$. As discussed, at the end of the setup phase, the $\Delta$ vector is generated. The $\Delta$ vector, therefore, is generated as $\Delta(2)=\left[\begin{array}{lllll}0 & 1 & 2 & 1 & 2\end{array}\right]^{T}$ and $\Delta(8)=\left[\begin{array}{lllll}0 & 0 & 0 & 1 & 1\end{array}\right]^{T}$ at round $r_{2}$ and $r_{8}$ respectively.

Similar to the previous simulation, we assume that each SU can accomplish a sensing accuracy that might be different from other SUs. Let $n_{e}(i)$ denote the total number of errors between the $i^{\text {th }}$ SU's sensing result and PU's ON-OFF state ${ }^{3}$. Without loss of generality, we assume that $n_{e}=[0,1,3,4,6]$. Secondary users are then classified based on their sensing accuracy into three groups: good $\left(S U_{1}\right.$ and $\left.S U_{2}\right)$, average $\left(S U_{3}\right.$ and $\left.S U_{4}\right)$, and poor $\left(S U_{5}\right)$.

In the operation phase, first the weighting function and $P$ matrices are calculated. We use $\eta_{i}^{x}$ defined in (5.22), and emphasize the sensing accuracy $(x=5, g=20)$. Second, $N^{*}=2$ secondary users that have the greatest $P$ values are selected to perform the sensing/reporting tasks. Fig. 5.16 depicts the above matrices, where $N^{*}=2$ secondary users that are selected are indicated by an "**" sign. For instance, $\left(S U_{1}\right.$ and $\left.S U_{2}\right)$ are selected in round $r_{3}$, and $\left(S U_{1}\right.$ and $\left.S U_{4}\right)$ are selected in round $r_{4}$. The selected $S U_{s}$ then

\footnotetext{
${ }^{3}$ Although the secondary network is not aware of the primary user's state, we use this information for the evaluation purpose.
} 


\begin{tabular}{|c|c|c|c|c|c|c|c|c|c|c|}
\hline \multirow[b]{2}{*}{ ROUND } & \multicolumn{2}{|c|}{ Setup Phase } & \multicolumn{4}{|c|}{ Operation Phase } & \multicolumn{2}{|c|}{ Setup Phase } & \multicolumn{2}{|c|}{ Operation Phase } \\
\hline & $r_{1}$ & $r_{2}$ & $r_{3}$ & $\mathrm{r}_{4}$ & $\mathrm{r}_{5}$ & $\mathrm{r}_{6}$ & $\mathrm{r}_{7}$ & $\mathrm{r}_{8}$ & $r_{9}$ & $r_{10}$ \\
\hline
\end{tabular}

\begin{tabular}{|l||l|l|l|l|l|l|l|l|l|l|}
\hline $\mathrm{PU}$ & 1 & 1 & 0 & 0 & 0 & 1 & 1 & 0 & 1 & 1 \\
\hline
\end{tabular}

\begin{tabular}{|l||c|c|c|c|c|c|c|c|c|c|}
\hline FC (OR-Rule) & 1 & 1 & 0 & 0 & 1 & 1 & 1 & 0 & 1 & 1 \\
\hline \hline SU 1 & 1 & 1 & 0 & 0 & 0 & 1 & 1 & 0 & $\mathrm{X}$ & $\mathrm{X}$ \\
\hline SU 2 & 0 & 1 & 0 & $\mathrm{X}$ & 0 & $\mathrm{X}$ & 1 & 0 & 1 & 1 \\
\hline SU 3 & 0 & 0 & $\mathrm{X}$ & $\mathrm{X}$ & $\mathrm{X}$ & $\mathrm{X}$ & 1 & 0 & 1 & 1 \\
\hline SU 4 & 1 & 0 & $\mathrm{X}$ & 0 & $\mathrm{X}$ & 0 & 0 & 0 & $\mathrm{X}$ & $\mathrm{X}$ \\
\hline SU 5 & 0 & 0 & $\mathrm{X}$ & $\mathrm{X}$ & $\mathrm{X}$ & $\mathrm{X}$ & 0 & 0 & $\mathrm{X}$ & $\mathrm{X}$ \\
\hline
\end{tabular}

Figure 5.15: The operation of the proposed method is divided into the setup phase and the operational phase. An "X" indicates that the corresponding SU does not perform senseing/reporting, a "1" means the PU is detected, and a " 0 " means the PU is not detected.

report their local sensing results to the FC. Third, the final decision is made by the FC according to the received sensing reports. Fig. 5.15 shows the sensing results obtained by the selected SUs, where an "X" indicates that non-selected SUs do not perform the sensing/reporting tasks.

In addition, each time an $\mathrm{SU}$ is selected, its energy level will be reduced by $e_{u}$. Fig. 5.16 depicts the energy level of each $\mathrm{SU}$ in the $e$ matrix. As shown, during the early rounds, the difference in $\eta^{x}$ values is mostly due to the sensing accuracy, because all SUs start with the same level of energy $\left(e_{0}\right)$. However, after a few rounds, when the energy level of SUs is not balanced any more (SUs that have better sensing accuracy have been selected more often), the selection of SUs is performed due to both sensing accuracy $\Delta_{i}(n)$ and $e_{i}(n)$, as discussed in (5.22). 


\begin{tabular}{|c|c|c|c|c|c|c|c|c|c|c|c|}
\hline & & & & Operati & Phase & & & & Operati & Phase & \\
\hline & $r_{1}$ & $\mathrm{r}_{2}$ & $r_{3}$ & $\mathrm{r}_{4}$ & $r_{5}$ & $r_{6}$ & $r_{7}$ & $\mathrm{r}_{8}$ & $r_{9}$ & $\mathrm{r}_{10}$ & \\
\hline & 0 & 0 & 0.0385 & 0.0338 & 0.0291 & 0.0244 & 0 & 0 & 0.0099 & 0.0099 & $\longleftarrow \mathrm{SU}_{1}$ \\
\hline & 0 & 0 & 0.0079 & 0.0077 & 0.0077 & 0.0074 & 0 & 0 & 0.0196 & 0.0148 & $\longleftarrow \mathrm{SU}_{2}$ \\
\hline$\eta=$ & 0 & 0 & 0.0044 & 0.0044 & 0.0044 & 0.0044 & 0 & 0 & 0.0291 & 0.0244 & $\longleftarrow \mathrm{SU}_{3}$ \\
\hline & 0 & 0 & 0.0079 & 0.0079 & 0.0077 & 0.0077 & 0 & 0 & 0.0066 & 0.0066 & $\longleftarrow \mathrm{SU}_{4}$ \\
\hline & 0 & 0 & 0.0044 & 0.0044 & 0.0044 & 0.0044 & 0 & 0 & 0.0074 & 0.0074 & $\longleftarrow \mathrm{SU}_{5}$ \\
\hline & 0 & 0 & $0.6087 *$ & $0.5798 *$ & $0.5453^{*}$ & $0.5039 *$ & 0 & 0 & 0.1362 & 0.1568 & $\longleftarrow \mathrm{SU}_{1}$ \\
\hline & 0 & 0 & $0.1256^{*}$ & 0.1323 & $0.1445^{*}$ & 0.1538 & 0 & 0 & $0.2697 *$ & $0.2341^{*}$ & $\longleftarrow \mathrm{SU}_{2}$ \\
\hline $\mathrm{P}=$ & 0 & 0 & 0.07 & 0.0759 & 0.0828 & 0.0914 & 0 & 0 & $0.4006^{*}$ & $0.3863^{*}$ & $\longleftarrow \mathrm{SU}_{3}$ \\
\hline & 0 & 0 & 0.1256 & $0.1361 *$ & 0.1445 & $0.1595 *$ & 0 & 0 & 0.0911 & 0.1049 & $\longleftarrow \mathrm{SU}_{4}$ \\
\hline & 0 & 0 & 0.07 & 0.0759 & 0.0828 & 0.0914 & 0 & 0 & 0.1024 & 0.1179 & $\longleftarrow \mathrm{SU}_{5}$ \\
\hline & 0.9 & 0.8 & 0.7 & 0.6 & 0.5 & 0.4 & 0.3 & 0.2 & 0.2 & 0.2 & $\longleftarrow \mathrm{SU}_{1}$ \\
\hline & 0.9 & 0.8 & 0.7 & 0.7 & 0.6 & 0.6 & 0.5 & 0.4 & 0.3 & 0.2 & $\longleftarrow \mathrm{SU}_{2}$ \\
\hline $\mathrm{e}=$ & 0.9 & 0.8 & 0.8 & 0.8 & 0.8 & 0.8 & 0.7 & 0.6 & 0.5 & 0.4 & $\longleftarrow \mathrm{SU}_{3}$ \\
\hline & 0.9 & 0.8 & 0.8 & 0.7 & 0.7 & 0.6 & 0.5 & 0.4 & 0.4 & 0.4 & $\longleftarrow \mathrm{SU}_{4}$ \\
\hline & 0.9 & 0.8 & 0.8 & 0.8 & 0.8 & 0.8 & 0.7 & 0.6 & 0.6 & 0.6 & $\longleftarrow \mathrm{SU}_{5}$ \\
\hline
\end{tabular}

Figure 5.16: Illustration of the weighting factor $\eta$, the probability matrix $P$, and the energy consumption matrix $e$ for rounds $1 \leq r \leq 10$. An "** in $P$ matrix indicates the active SUs at each round. 
As shown in Fig. 5.16, during rounds $r_{3}-r_{6}$ and $r_{9}-r_{10}, S U_{1}, S U_{2}, S U_{3}, S U_{4}$, and $S U_{5}$ are selected 4, 4, 2, 2, and 0 times respectively. In other words, the selection process took place 6 times, with 2 SUs selected each time (12 times in total). Out of the 12 selections, 8 times selected SUs belonged to the good sensing accuracy (66\%), 4 times selected SUs belonged to the average sensing accuracy (33\%), and 0 times selected SUs belonged to the poor sensing accuracy (0\%).

\subsection{Summary}

In this chapter, we reviewed cooperative sensing in cognitive sensor networks. An optimization problem was formulated and analytically solved to find the minimum number of required cooperative users. We then defined a metric for spectrum sensing accuracy and proposed a novel energy-aware user selection method that identifies the most eligible cooperative users through a probability-based approach that employs weighting functions.

In addition, two benchmarks were defined for the purpose of the proposed method's evaluation. We also compared the network lifetime for several sensing accuracy thresholds, and discussed the trade-off between the sensing accuracy and the network lifetime. Moreover, the effect of different fusion rules were discussed and it was concluded that the Majority rule has the best overall performance among the examined rules. The chapter 
ends with a numerical analysis to further enlighten the proposed method's approach to the cooperative spectrum sensing. 


\section{Chapter 6}

\section{Conclusions and Future Work}

\subsection{Concluding Remarks}

In this dissertation, we studied spectrum sensing in cognitive radio networks. We highlighted the importance of spectrum occupation modeling and described the spectrum usage in cognitive radio by a continuous-time Markov chain model. The model was mathematically defined by deriving the transition rate matrix and the probability state vector. Through numerical analyses, we validated the model for several cases including an extreme case with a very large number of secondary users. The model was also examined for a special case with no active secondary user and compared with an existing model in the literature; it was discussed that the model agrees with the existing work in this case. The Markov chain served as a base model for our analysis through this 
dissertation.

We elaborated the significance of sensing period optimization on the secondary network power consumption and throughput. We also discussed the current drawback in the most existing methods where the sensing period is obtained based on the primary network's properties only. This drawback is highlighted by knowing that there exist numerous cognitive radio applications with various sets of design requirements. On the other hand, a cognitive radio network should be aware of its surroundings, and the traffic characteristics of the secondary network are important, especially when it has exceptionally low or high probability of presence. We, therefore, proposed an application-specific spectrum sensing method that finds the local sensing period according to the properties of both primary and secondary networks. We defined and analytically derived the interference ratio and the undetected opportunity ratio parameters. Then, two optimization problems were formulated based on the secondary network's state in the Markov chain, and the optimum sensing period in each state was analytically derived.

In addition, we examined the secondary network's power consumption and its throughput in the application-specific and the non-application-specific sensing approaches. It was elaborated that while the non-application-specific method provides an identical solution for different types of secondary networks, the application-specific approach provides a customized solution for each network. The performance of these schemes were compared 
through various numerical and simulation analyses. It was concluded that, as compared with the non-application-specific approach, the proposed method managed to increase the average throughput of the secondary networks by up to $11 \%$. Also, the proposed method significantly decreased the secondary network's power consumption by as low as $33 \%$ of the non-hybrid approach. We also discussed the trade-off between the throughput maximization and the power consumption minimization.

We also investigated the cooperative spectrum sensing in cognitive radio and discussed the main advantages of cooperative techniques in spectrum sensing. We then addressed and analytically solved two important problems in the cooperative spectrum sensing: the minimum number of cooperative users and the most eligible cooperative users. We also defined a metric for the sensing accuracy and proposed a novel method that identifies the most eligible secondary users through a probability-based approach that uses a weighting function.

Two benchmarks were defined to examine the effects of emphasizing on the sensing accuracy and the remaining energy level of the secondary users. In particular, we studied two extreme cases where only one of the parameters was considered in each case. By numerical analysis, it was concluded that the accuracy benchmark was increased by as much as $39 \%$ by only considering the sensing accuracy and ignoring the remaining energy level. Additionally, the energy benchmark was reduced by as as low as $76 \%$ by 
only considering the remaining level of energy and ignoring the sensing accuracy. We also compared the network's lifetime at several sensing accuracy thresholds and discussed the trade-off between the sensing accuracy and the network's lifetime. Moreover, the effects of several fusion rules were studied, including the OR-Rule, the AND-Rule and the Majority rule. Through several simulations, it was concluded that the Majority rule had the best overall performance among the fusion rules. Finally, a numerical analysis was performed to further enlighten the proposed method's approach to the spectrum sensing.

\subsection{Future Directions}

The proposed sensing methods through this dissertation are based on a continuous-time Markov chain model that is developed as part of this work. The proposed sensing methods can be further enriched by assuming more sophisticated spectrum occupation models.

In addition, the current work assumes a fixed network structure where the location of the primary and secondary users does not vary by time. However, in reality, there exist a number of applications where the location of the users is not fixed. Therefore, it is essential to address the spectrum sensing optimization in cognitive radio networks with mobile users. Adding mobility to SUs can provide sensing diversity as a single SU might obtain the channel usage information from different geographical locations. The

probability of detection, therefore, is expected to be improved. A possible direction to 
reduce the complexity of sensing period optimization in a mobile cognitive radio network would be to estimate the channel usage information based on the previous sensing results. 


\section{References}

[1] H. Kim and K. Shin, "Efficient discovery of spectrum opportunities with MAClayer sensing in cognitive radio networks," IEEE Trans. Mobile Computing, vol. 7, pp. 533-545, May 2008.

[2] X. Zhu, L. Shen, and T.-S. Yum, "Analysis of cognitive radio spectrum access with optimal channel reservation," Communications Letters, IEEE, vol. 11, pp. $304-306$, Apr. 2007.

[3] S. M. M. D. Cabric and R. Brodersen, "Implementation issues in spectrum sensing for cognitive radios," in Proc. Asilomar Conf. Signals, Systems and Computers, pp. $772-776$, Nov. 2004.

[4] FCC, "Spectrum policy task force," in ET Docket No. 02- 135, Nov. 2002.

[5] I. Mitola, J. and J. Maguire, G.Q., "Cognitive radio: Making software radios more personal," Personal Communications, IEEE, vol. 6, pp. 13 -18, Aug. 1999.

[6] J. Mitola, Cognitive Radio: An Integrated Agent Architecture for Software Defined Radio. PhD thesis, Royal Inst. Technology, Stockholm, Sweden, 2000.

[7] S. Haykin, "Cognitive radio: Brain-empowered wireless communications," Selected Areas in Communications, IEEE Journal on, vol. 23, pp. 201- 220, Feb. 2005.

[8] M. C. V. I. F. Akyildiz, W. Lee and S. Mohanty, "A survey on spectrum management in cognitive radio networks," Communications Magazine, IEEE, vol. 46, pp. 40-48, Apr. 2008. 
[9] R. V. Prasad and H. S. B. P. Pawczak, James A. Hoffmeyer, "Cognitive functionality in next generation wireless networks: Standardization efforts," Communications Magazine, IEEE, vol. 46, pp. 72-78, Apr. 2008.

[10] W. Heinzelman, A. Chandrakasan, and H. Balakrishnan, "An application-specific protocol architecture for wireless microsensor networks," IEEE Trans. Wireless Communications, vol. 1, pp. 660 - 670, Oct. 2002.

[11] A. Zahmati, S. Hussain, X. Fernando, and A. Grami, "Cognitive wireless sensor networks: Emerging topics and recent challenges," in Proc. IEEE TIC-STH, pp. 593 -596 , Sept. 2009.

[12] N. Shankar, C. Cordeiro, and K. Challapali, "Spectrum agile radios: Utilization and sensing architectures," in Proc. IEEE DySPAN, pp. 160 -169, Nov. 2005.

[13] J. W. D. Cavalcanti, S. Das and K. Challapali, "Cognitive radio based wireless sensor networks," in Proc. IEEE ICCCN, pp. 1-6, Ieee, 2008.

[14] S. Gao, L. Qian, and D. Vaman, "Distributed energy efficient spectrum access in wireless cognitive radio sensor networks," in Proc. WCNC, pp. 1442 - 1447, Mar. 2008.

[15] S. Gao, L. Qian, D. Vaman, and Q. Qu, "Energy efficient adaptive modulation in wireless cognitive radio sensor networks," in Proc. IEEE ICC, pp. 3980 -3986, June 2007.

[16] S. Maleki, A. Pandharipande, and G. Leus, "Energy-efficient distributed spectrum sensing for cognitive sensor networks," Sensors Journal, IEEE, vol. 11, pp. $565-573$, Mar. 2011.

[17] I. F. Akyildiz, W. Lee, M. C. Vuran, and S. Mohanty, "Next generation/dynamic spectrum access/cognitive radio wireless networks: A survey," Computer Networks, Elsevier, vol. 50, pp. 2127-2159, Sept. 2006. 
[18] F. K. Jondral, "Software-defined radio - basic and evolution to cognitive radio," Wireless Commun. and Networking, EURASIP, 2005.

[19] A. Ghasemi and E. Sousa, "Spectrum sensing in cognitive radio networks: Requirements, challenges and design trade-offs," Communications Magazine, IEEE, vol. 46, pp. $32-39$, Apr. 2008.

[20] N. H. A. Sahai and R. Tandra, "Some fundamental limits on cognitive radio," in Proc. Allerton Conference on Communication, Control, and Computing, pp. 1662$1671,2004$.

[21] A. Ghasemi and E. Sousa, "Collaborative spectrum sensing for opportunistic access in fading environments," in Proc. IEEE DySPAN, pp. 131 -136, Nov. 2005.

[22] H. Tang, "Some physical layer issues of wide-band cognitive radio systems," in Proc. IEEE DySPAN, pp. 151 -159, Nov. 2005.

[23] A. Fehske, J. Gaeddert, and J. Reed, "A new approach to signal classification using spectral correlation and neural networks," in Proc. IEEE DySPAN, pp. $144-150$, Nov. 2005.

[24] J. Zhao, H. Zheng, and G.-H. Yang, "Distributed coordination in dynamic spectrum allocation networks," in Proc. IEEE DySPAN, pp. 259 -268, Nov. 2005.

[25] H. Zheng and L. Cao, "Device-centric spectrum management," in Proc. IEEE DySPAN, pp. $56-65$, Nov. 2005.

[26] IEEE, "IEEE 802.22 WG on wireless regional area networks," http://www.ieee802.org/22.

[27] W. Yu-chun, W. Haiguang, and P. Zhang, "Protection of wireless microphones in IEEE 802.22 cognitive radio network," in Proc. IEEE ICC Workshops, pp. 1 -5, June 2009 . 
[28] FCC, "Unlicensed operations in the tv broadcast bands, second memorandum opinion and order, document number: 2010-30184," Dec. 2010.

[29] O. Akan, O. Karli, and O. Ergul, "Cognitive radio sensor networks," Network, IEEE, vol. 23, pp. $34-40$, Jul.-Aug. 2009.

[30] K. Shenai and S. Mukhopadhyay, "Cognitive sensor networks," in Proc. International Conference on Microelectronics, pp. 315 -320, May 2008.

[31] P. Boonma and J. Suzuki, "Exploring self-star properties in cognitive sensor networking," in Proc. International Symposium on Performance Evaluation of Computer and Telecommunication Systems, pp. 36 -43, June 2008.

[32] S. Gao, L. Qian, D. Vaman, and Q. Qu, "Energy efficient adaptive modulation in wireless cognitive radio sensor networks," in Proc. IEEE ICC, pp. 3980 -3986, June 2007.

[33] ECMA, "Ecma 392: MAC and PHY for operation in TV white space," Tech. report, 2nd ed., June 2012.

[34] M. Mueck, A. Piipponen, K. Kallioja andrvi, G. Dimitrakopoulos, K. Tsagkaris, P. Demestichas, F. Casadevall, J. Pe andrez Romero, O. Sallent, G. Baldini, S. Filin, H. Harada, M. Debbah, T. Haustein, J. Gebert, B. Deschamps, P. Bender, M. Street, S. Kandeepan, J. Lota, and A. Hayar, "Etsi reconfigurable radio systems: Status and future directions on software defined radio and cognitive radio standards," Communications Magazine, IEEE, vol. 48, pp. 78 -86, Sept. 2010.

[35] M. McHenry, "NSF spectrum occupancy measurements project summary," Shared spectrum company report, 2005.

[36] "IEEE standard for information technology-telecommunications and information exchange between systems wireless regional area networks (WRAN)-specific requirements part 22: Cognitive wireless RAN medium access control (MAC) and physical 
layer (PHY) specifications: Policies and procedures for operation in the tv bands," IEEE Std 802.22-2011, pp. 1 -680, Jan. 2011.

[37] C. Cordeiro, K. Challapali, D. Birru, and N. Sai Shankar, "IEEE 802.22: The first worldwide wireless standard based on cognitive radios," in Proc. DySPAN, pp. 328337, Nov. 2005.

[38] E. S. C. R. Stevenson, C. Cordeiro and G. Chouinard, "Functional requirements for the 802.22 WRAN standard," IEEE 802.22-05/000\%r46, Sept. 2005.

[39] J. Wang, M. S. Song, S. Santhiveeran, K. Lim, G. Ko, K. Kim, S. H. Hwang, M. Ghosh, V. Gaddam, and K. Challapali, "First cognitive radio networking standard for personal/portable devices in tv white spaces," in Proc. IEEE DySPAN, pp. 1 -12, Apr. 2010.

[40] C. Ghosh, C. Cordeiro, D. Agrawal, and M. Rao, "Markov chain existence and hidden markov models in spectrum sensing," in Proc. IEEE PerCom, pp. 1 -6, Mar. 2009.

[41] L. Luo and S. Roy, "A two-stage sensing technique for dynamic spectrum access," in Proc. IEEE ICC, pp. 4181 -4185, May 2008.

[42] T. Rondeau, C. Rieser, T. Gallagher, and C. Bostian, "Online modeling of wireless channels with hidden markov models and channel impulse responses for cognitive radios," in Proc. IEEE MTT-S, vol. 2, pp. 739 - 742 Vol.2, June 2004.

[43] S.-M. L. C-H. Park, S-W. Kim and M.-S. Song, "HMM based channel status predictor for cognitive radio," in Proc. IEEE Asia-Pacific Microwave Conference, pp. 1-4, Dec. 2007.

[44] I. Akbar and W. Tranter, "Dynamic spectrum allocation in cognitive radio using hidden markov models: Poisson distributed case," in Proc. IEEE SoutheastCon, pp. 196 -201, Mar. 2007. 
[45] H. Kim and K. G. Shin, "Adaptive mac-layer sensing of spectrum availability in cognitive radio networks," Tech. Report, Real-Time Computing Laboratory, University of Michigan, 2006.

[46] H. Kim and K. Shin, "Fast discovery of spectrum opportunities in cognitive radio networks," in Proc. IEEE DySPAN, pp. 1-12, Oct. 2008.

[47] L. T. S. Geirhofer and B. M. Sadler, "Dynamic spectrum access in the time domain: Modeling and exploiting white space," Communications Magazine, IEEE, vol. 45, pp. 66-72, May 2007.

[48] S. Geirhofer, L. Tong, and B. Sadler, "Cognitive medium access: Constraining interference based on experimental models," Selected Areas in Communications, IEEE Journal on, vol. 26, pp. $95-105$, Jan. 2008.

[49] L. T. S. Geirhofer and B. M. Sadler, "A measurement-based model for dynamic spectrum access," in Proc. IEEE MILCOM, vol. 26, 2006.

[50] A. Min, K.-H. Kim, J. Singh, and K. Shin, "Opportunistic spectrum access for mobile cognitive radios," in Proc. IEEE INFOCOM, pp. 2993 -3001, April 2011.

[51] B. Wang, Z. Ji, K. Liu, and T. Clancy, "Primary-prioritized markov approach for dynamic spectrum allocation," IEEE Trans. Wireless Communications, vol. 8, pp. 1854 -1865, Apr. 2009.

[52] Y. Xing, R. Chandramouli, S. Mangold, and S. N, "Dynamic spectrum access in open spectrum wireless networks," Selected Areas in Communications, IEEE Journal on, vol. 24, pp. 626 - 637, Mar. 2006.

[53] C. G. Cassandras and S. Lafortune, Introduction to discrete event systems, vol. 11. Kluwer academic publishers, 1999.

[54] J. N. Daigle, Queueing theory with applications to packet telecommunication. Springer, 2005. 
[55] V. G. Kulkarni, Modeling and analysis of stochastic systems. CRC Press, 1995.

[56] H. d. M. G. Bolch, S. Greiner and K. S. Trivedi, Queueing networks and Markov chains: Modeling and performance evaluation with computer science applications. John Wiley and Sons, 2006.

[57] J. Wang, M. Ghosh, and K. Challapali, "Emerging cognitive radio applications: A survey," Communications Magazine, IEEE, vol. 49, pp. 74 -81, Mar. 2011.

[58] Z. Liang, S. Feng, D. Zhao, and X. Shen, "Delay performance analysis for supporting real-time traffic in a cognitive radio sensor network," IEEE Trans. Wireless Communications, vol. 10, pp. 325 -335, Jan. 2011.

[59] Y. Chen, W. L. Woo, and C.-X. Wang, "Channel modeling of information transmission over cognitive interrogator-sensor networks," IEEE Trans. Vehicular Technology, vol. 60, pp. $2-15$, Jan. 2011.

[60] O. Elgezabal and C. Salazar, "Technological foundation for future intra-aircraft wireless applications: Technology assessment," in Proc. IEEE FBW, pp. 1 -19, June 2011.

[61] E. Peh, Y.-C. Liang, Y. L. Guan, and Y. Zeng, "Optimization of cooperative sensing in cognitive radio networks: A sensing-throughput tradeoff view," IEEE Trans. Vehicular Technology, vol. 58, pp. 5294 - 5299, Nov. 2009.

[62] S. S. Jeong, W. S. Jeon, and D. G. Jeong, "Collaborative spectrum sensing for multiuser cognitive radio systems," IEEE Trans. Vehicular Technology, vol. 58, pp. 2564 -2569 , Jun. 2009.

[63] J. Salt and H. Nguyen, "Performance prediction for energy detection of unknown signals," IEEE Trans. Vehicular Technology, vol. 57, pp. 3900 - 3904, Nov. 2008. 
[64] Y. C. Liang, Y. Zeng, E. Peh, and A. T. Hoang, "Sensing-throughput tradeoff for cognitive radio networks," IEEE Trans. Wireless Communications, vol. 7, pp. 1326 -1337, Apr. 2008.

[65] G. Ganesan and Y. Li, "Agility improvement through cooperative diversity in cognitive radio," in Proc. IEEE GLOBECOM, vol. 5, pp. 2505 -2509, Dec. 2005.

[66] T. Yucek and H. Arslan, "A survey of spectrum sensing algorithms for cognitive radio applications," Communications Surveys Tutorials, IEEE, vol. 11, pp. 116 130, Quarter 2009.

[67] F. Digham, M.-S. Alouini, and M. Simon, "On the energy detection of unknown signals over fading channels," in Proc. IEEE ICC, vol. 5, pp. 3575 - 3579, May 2003.

[68] H. Tang, "Some physical layer issues of wide-band cognitive radio systems," in Proc. IEEE DySPAN, pp. $151-159$, Nov. 2005.

[69] T. M. Cover and J. A. Thomas, Elements of Information Theory. New York: WileyInterscience, 1990.

[70] W. B. Chien, C. K. Yang, and Y. H. Huang, "Energy-saving cooperative spectrum sensing processor for cognitive radio system," IEEE Trans. Circuits and Systems I: Regular Papers, vol. 58, pp. 711 -723, Apr. 2011.

[71] J. A. Han, W. S. Jeon, and D. G. Jeong, "Energy-efficient channel management scheme for cognitive radio sensor networks," IEEE Trans. Vehicular Technology, vol. 60, pp. 1905 -1910, May 2011.

[72] R. Deng, J. Chen, C. Yuen, P. Cheng, and Y. Sun, "Energy-efficient cooperative spectrum sensing by optimal scheduling in sensor-aided cognitive radio networks," IEEE Trans. Vehicular Technology, vol. 61, pp. 716 -725, Feb. 2012. 
[73] Z. Quan, S. Cui, A. Sayed, and H. Poor, "Optimal multiband joint detection for spectrum sensing in cognitive radio networks," IEEE Trans. Signal Processing, vol. 57, pp. $1128-1140$, Mar. 2009.

[74] Y. Rao, W. Chen, and Z. Cao, "A sequential sensing data transmission and fusion approach for large scale cognitive radios," in Proc. IEEE ICCE, pp. 1-5, May 2010.

[75] W.-Y. Lee and I. Akyildiz, "Optimal spectrum sensing framework for cognitive radio networks," IEEE Trans. Wireless Communications, vol. 7, pp. 3845 -3857, Oct. 2008.

[76] R. Viswanathan and P. Varshney, "Distributed detection with multiple sensors I. fundamentals," Proceedings of the IEEE, vol. 85, pp. 54 -63, Jan. 1997.

[77] F. Digham, M.-S. Alouini, and M. Simon, "On the energy detection of unknown signals over fading channels," in Proc. IEEE ICC, vol. 5, pp. 3575 - 3579 vol.5, May 2003.

[78] W. Zhang, R. Mallik, and K. Letaief, "Optimization of cooperative spectrum sensing with energy detection in cognitive radio networks," IEEE Trans. Wireless Communications, vol. 8, pp. 5761 -5766, Dec. 2009.

[79] N. M. Temme, "Uniform asymptotic expansions of the incomplete gamma functions and the incomplete beta function," Mathematics of Computation, vol. 29, pp. 11091114, Oct. 1975. 